\title{
Recoding of bacteriophage T4 gene 60 mRNA by programmed translational bypassing
}

\author{
Dissertation \\ for the award of the degree \\ “Doctor rerum naturalium” (Dr. rer. nat.) \\ of the Georg-August-Universität Göttingen
}

within the doctoral program: GGNB Biomolecules: Structure - Function - Dynamics

of the Georg-August University School of Science (GAUSS)

\author{
submitted by \\ Mariia Klimova \\ from Moscow, Russian Federation
}

Göttingen, 2020 



\section{Thesis Committee:}

Prof. Dr. Marina V. Rodnina

Department of Physical Biochemistry

Max Planck Institute for Biophysical Chemistry, Göttingen, Germany

Prof. Dr. Holger Stark

Department of Structural Dynamics

Max Planck Institute for Biophysical Chemistry, Göttingen, Germany

Prof. Dr. Heike Krebber

Department of Molecular Genetic

Institute for Microbiology and Genetics, Göttingen, Germany

Members of the Examination Board:

Reviewer: Prof. Dr. Marina V. Rodnina

Department of Physical Biochemistry

Max Planck Institute for Biophysical Chemistry, Göttingen, Germany

Second reviewer: Prof. Dr. Kai Tittmann

Department of Molecular Enzymology

Georg August University Göttingen, Göttingen, Germany

Further members of the Examination Board:

Prof. Dr. Ralf Ficner

Department of Molecular Structural Biology

Institute for Microbiology and Genetics, Göttingen, Germany

Prof. Dr. Wolfgang Wintermeyer

Research Group of Ribosome Dynamics

Max Planck Institute for Biophysical Chemistry, Göttingen, Germany

Date of oral examination: 10th of February 2020 



\section{Table of contents}

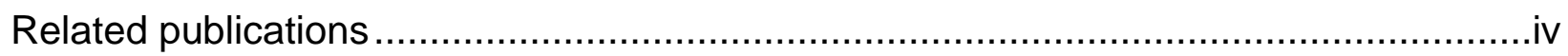

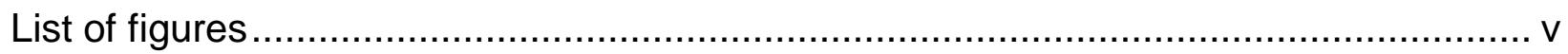

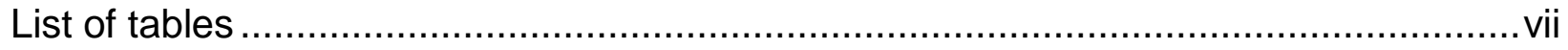

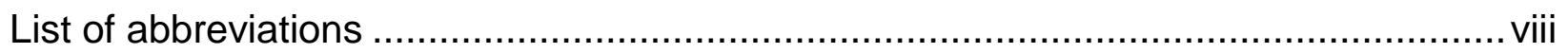

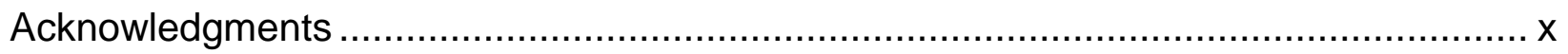

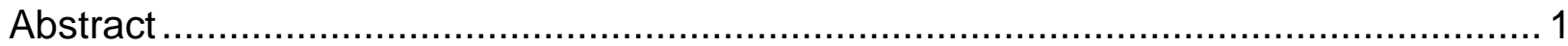

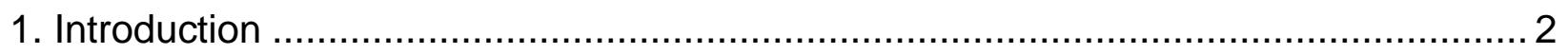

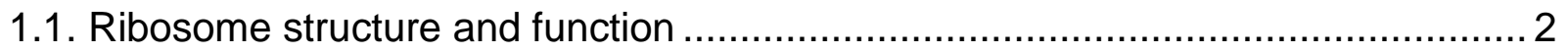

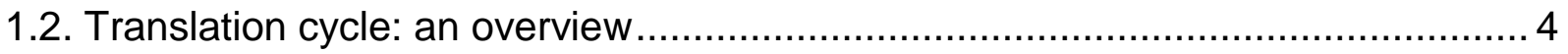

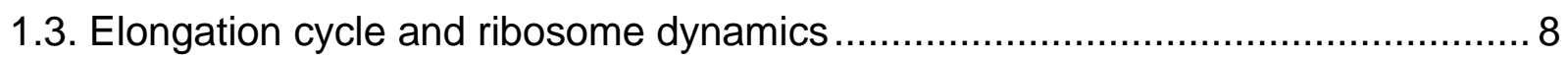

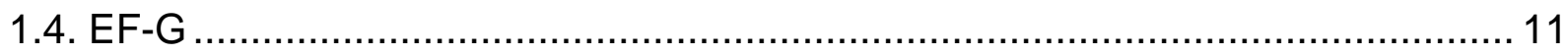

1.5. Major determinants of the translation rate ................................................. 14

1.5.1. Translation rate modulated by the nascent polypeptide ............................. 14

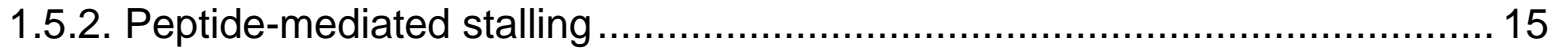

1.5.3. Connection between the mRNA secondary structure and translation rate ... 16

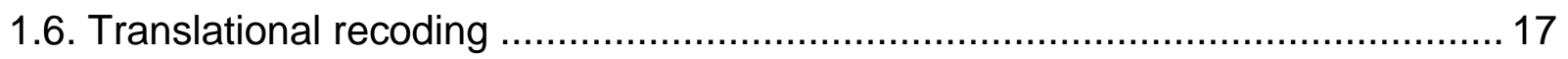

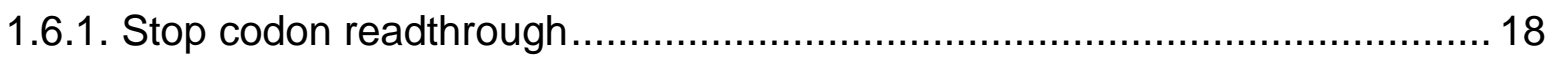

1.6.2. Programmed ribosomal frameshifting .................................................. 19

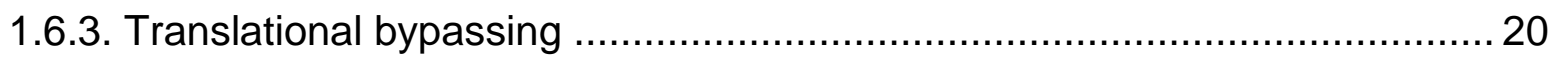

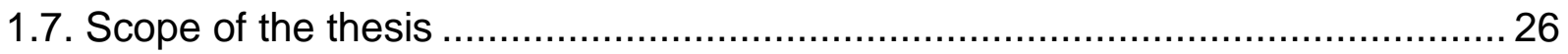

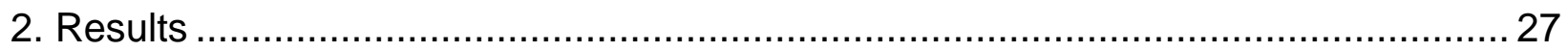

2.1. In vitro reconstituted $E$. coli translation system and product analysis ................ 27

2.2. Studying the effect of elongation factors on bypassing initiation....................... 28

2.3. Monitoring bypassing initiation using a $\left[{ }^{14} \mathrm{C}\right]$ Leu incorporation assay ................. 31

2.4. GTP expenditure during ribosomal sliding on the non-coding gap...................... 34

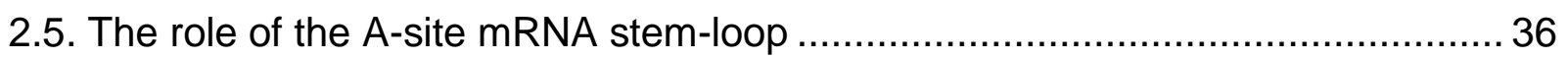

2.6. Conformational dynamics of the decoding center as a bypassing modulator ..... 38

2.7. Ribosome dynamics as a modulator of bypassing efficiency ........................... 43

2.8. Dynamics of ribosomal subunits during bypassing initiation and sliding ..............50 
2.9. The effect of ribosomal protein L9 on programmed bypassing in vitro. 57

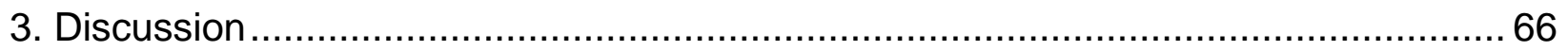

3.1. EF-G-induced ribosome sliding along the noncoding mRNA ...........................66

3.2. The role of ribosomal protein L9 in programmed bypassing in vitro.................... 71

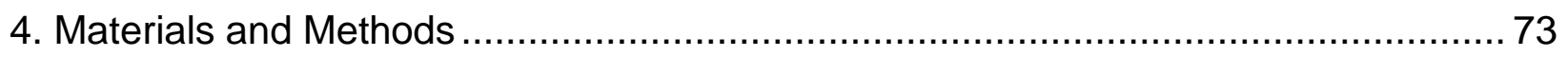

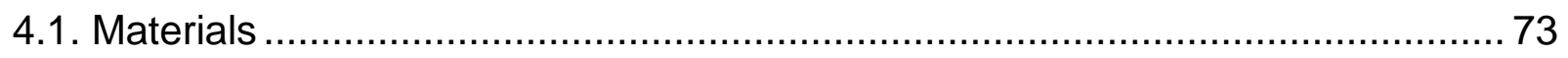

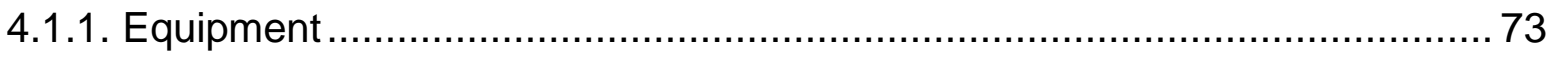

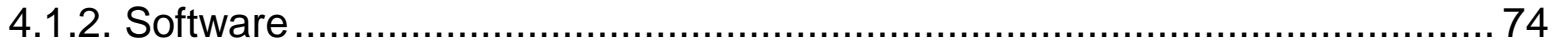

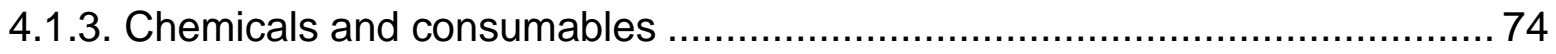

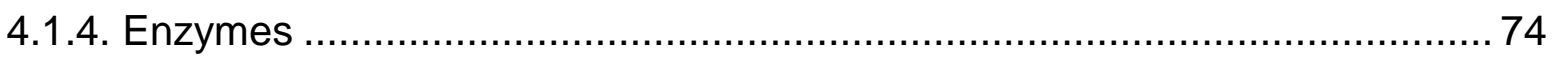

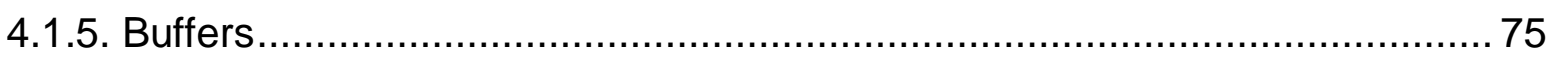

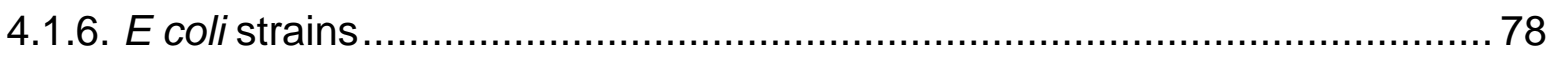

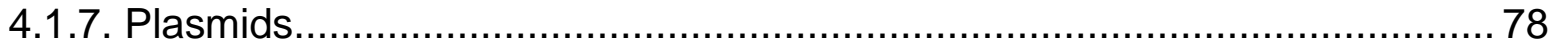

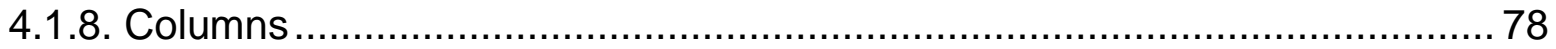

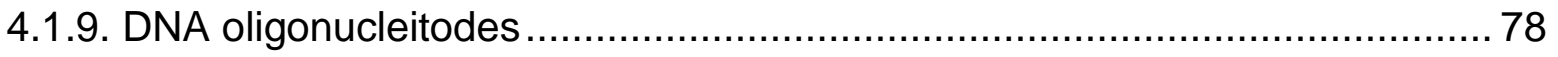

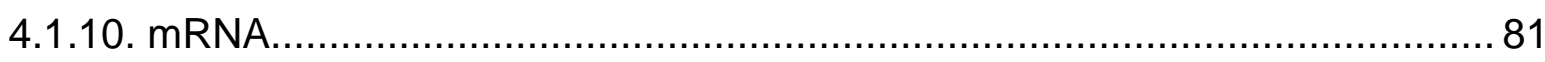

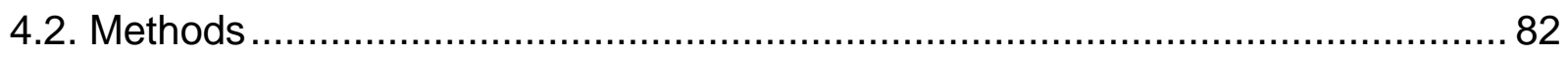

4.2.1. Template pDNA construction by polymerase chain reaction (PCR) ............. 82

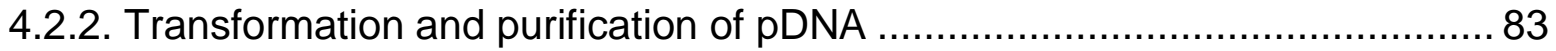

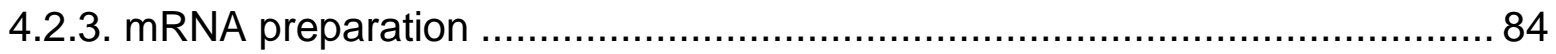

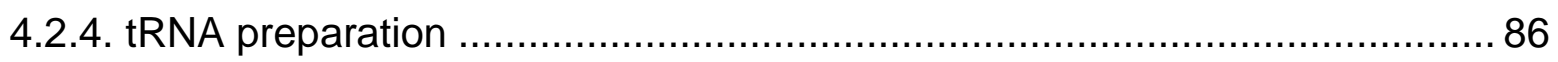

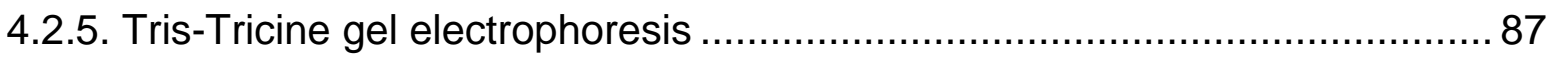

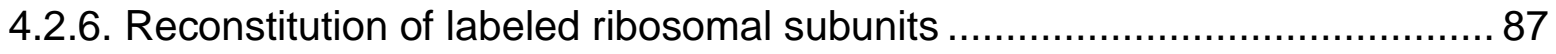

4.2.7. Initiation complex formation (non-labeled ribosomes) ................................ 88

4.2.8. Initiation complex formation (labeled ribosomes) ...................................... 88

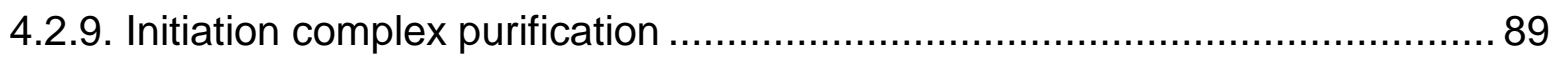

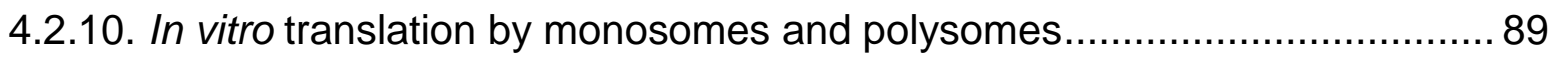

4.2.11. In vitro translation at the different temperatures ....................................... 90

4.2.12. Stalled take-off complexes ................................................................ 90

4.2.13. Leu incorporation into the nascent peptide............................................ 90

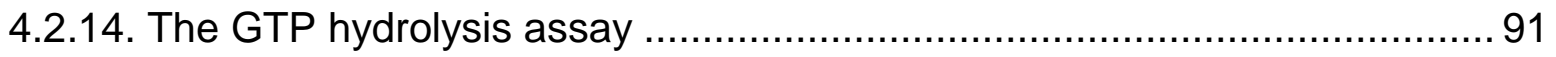

4.2.15. Time resolved puromycin assay ............................................................. 91 


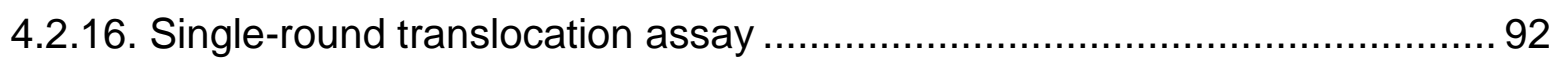

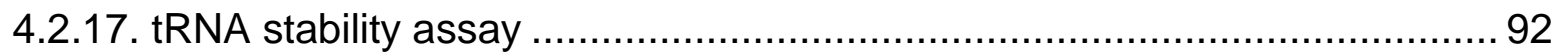

4.2.18. Single-molecule experiments using TIRF microscopy............................ 92

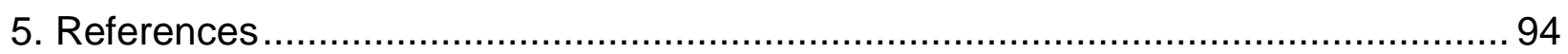

Curriculum Vitae ...................................................... Error! Bookmark not defined. 


\section{Related publications}

1. Klimova, M., Senyushkina, T., Samatova, E., Peng, B. Z., Pearson, M., Peske, F., \& Rodnina, M. V. (2019). EF-G-induced ribosome sliding along the noncoding mRNA. Science Advances. https://doi.org/10.1126/sciadv.aaw9049

2. Rodnina, M. V, Korniy, N., Klimova, M., Karki, P., Peng, B.-Z., Senyushkina, T., ... Peske, F. (2019). Translational recoding: canonical translation mechanisms reinterpreted. Nucleic Acids Research. https://doi.org/10.1093/nar/gkz783

3. Agirrezabala, X., Samatova, E., Klimova, M., Zamora, M., Gil-Carton, D., Rodnina, M. V., \& Valle, M. (2017). Ribosome rearrangements at the onset of translational bypassing. Science Advances, 3(6), 1-9. https://doi.org/10.1126/sciadv.1700147 


\section{List of figures}

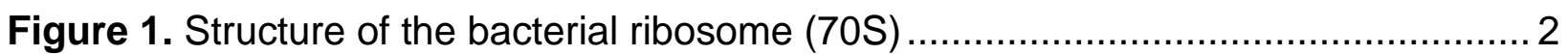

Figure 2. Conformational dynamics of the ribosome ............................................. 11

Figure 3. Different EF-G conformations during translocation................................... 13

Figure 4. Programmed recoding events ........................................................ 18

Figure 5. Schematic overview of the bypassing elements in the mRNA of bacteriophage

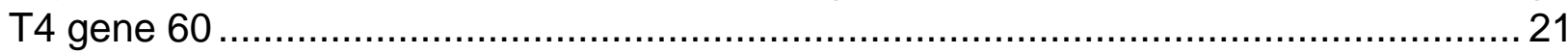

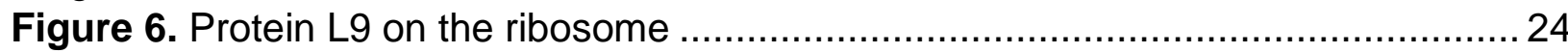

Figure 7. Formation of peptide products upon translation of gene 60 mRNA at the

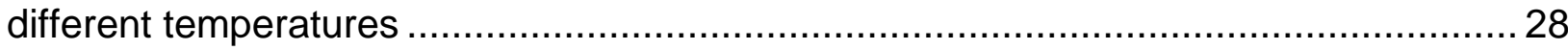

Figure 8. Translation and bypassing at different EF-G concentrations ....................... 30

Figure 9. Translation and bypassing at different EF-G and EF-Tu concentrations ........ 31

Figure 10. Identification of the factor essential for bypassing ................................... 33

Figure 11. Time course of GTP hydrolysis during bypassing …............................... 35

Figure 12. Effect of mutations in the A-site SL on bypassing ….............................. 37

Figure 13. The effect of mutations in the A-site $S L$ on temperature dependence of

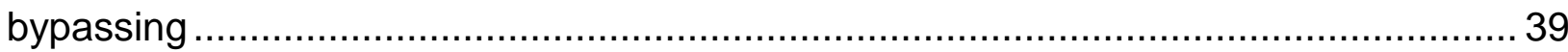

Figure 14. The effect of mutations in the 5'SL on temperature dependence of bypassing

Figure 15. The effect of mutations in the nascent peptide on temperature dependence of bypassing .................................................................................................. 42

Figure 16. Close-up of the decoding region of the small ribosomal subunit ................ 44

Figure 17. Functional characterization of the ribosomes with mutations in $\mathrm{S} 12$ in comparison with the WT ribosomes

Figure 18. Bypassing of ribosomes carrying mutations in S12 and EF-G with mutations in the domain IV

Figure 19. Bypassing of ribosomes carrying mutations in S12 combined with EF-G with

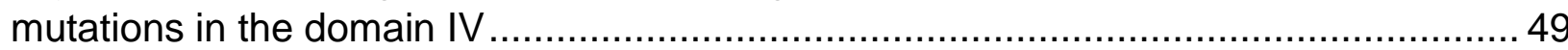

Figure 20. Summary of $T_{b}$ for the combinations of WT and mutant S12 and EF-G ......50

Figure 21. Bypassing activity of fluorescence-labeled ribosomal subunits ...................51

Figure 22. Ribosomal conformations during bypassing on gene 60 mRNA visualized by smFRET

Figure 23. Ribosomal conformations during bypassing on different gene $60 \mathrm{mRNA}$ constructs visualized by smFRET

Figure 24. Distribution of hyper-rotated, rotated, and non-rotated ribosomal populations in different complexes normalized to the activity of the take-off complex .....................55 Figure 25. Ribosomal conformations during SecM stalling visualized by smFRET ......56 Figure 26. Initiation efficiency for intact (WT) and mutant $(\Delta \mathrm{L} 9)$ ribosomes on the gene

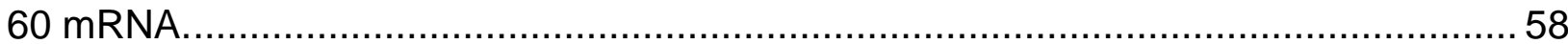

Figure 27. Effect of L9 deletion on the temperature dependence of bypassing ............58 
Figure 28. Relative bypassing efficiencies of WT and $\Delta \mathrm{L} 9$ ribosomes on different gene 60 mRNA constructs

Figure 29. Effect of mutations in the nascent peptide on the bypassing efficiency of WT and $\Delta \mathrm{L} 9$ ribosomes 60

Figure 30. The effect of polysome formation on bypassing efficiency for WT ribosomes 62

Figure 31. The effect of polysome formation on bypassing efficiency for $\Delta L 9$ ribosomes

Figure 32. The proposed mechanism of bypassing 64 69 


\section{List of tables}

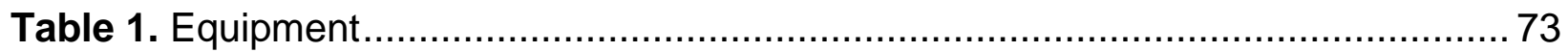

Table 2. Commercial software packages utilized in the work.................................. 74

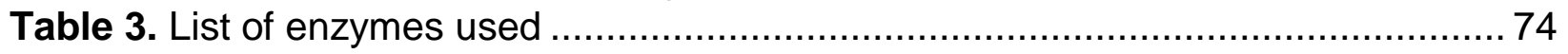

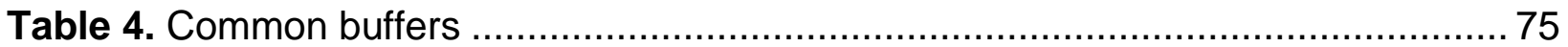

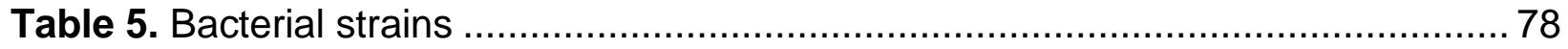

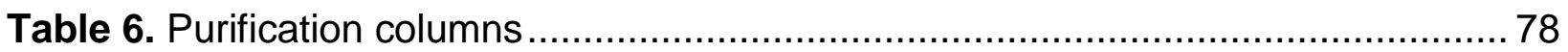

Table 7. Primers for gene 60 constructs (IBA Lifesciences or Eurofins Genomics) ...... 78

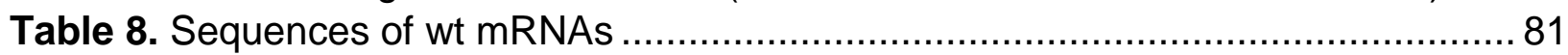

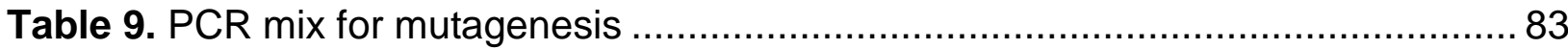

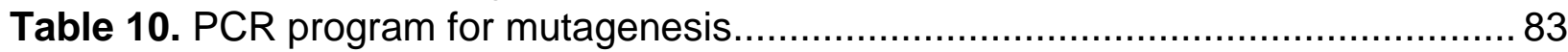

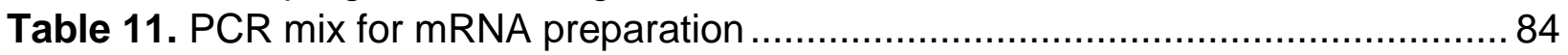

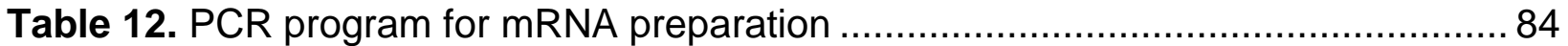

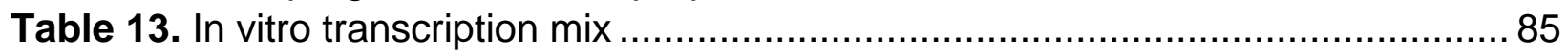

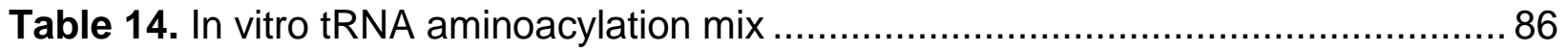




\section{List of abbreviations}

$\begin{array}{ll}\text { A site } & \text { Aminoacyl site } \\ \text { A260 } & \text { Absorbance at 260 nm } \\ \text { aa-tRNA } & \text { Aminoacyl-tRNA } \\ \text { APS } & \text { Ammonium persulfate } \\ \text { ATP } & \text { Adenosine triphosphate } \\ \text { BODIPY } & \text { Boron-dipyrromethene } \\ \text { CCW } & \text { Counterclockwise } \\ \text { Cryo-EM, EM } & \text { Electron cryomicroscopy } \\ \text { CW } & \text { Clockwise } \\ \text { DNA } & \text { Deoxyribonucleic acid } \\ \text { dNTP } & \text { (deoxy)nucleoside triphosphate } \\ \text { dpm } & \text { Disintegrations per minute } \\ \text { DTT } & \text { Dithiothreitol } \\ \text { E site } & \text { Ribosomal exit site } \\ \text { e.g. } & \text { (exempli gratia) for example } \\ \text { EF-G } & \text { Elongation factor G } \\ \text { EF-Tu } & \text { Elongation factor Tu } \\ \text { FPLC } & \text { Fast protein liquid chromatography } \\ \text { FRET } & \text { Förster resonance energy transfer } \\ \text { g } & \text { Gram } \\ \text { GDP } & \text { Guanosine diphosphate } \\ \text { GTP } & \text { Guanosine triphosphate } \\ \text { h } & \text { Hour } \\ \text { HPLC } & \text { High pressure liquid chromatography } \\ \text { i.e. } & \text { (id est) that is } \\ \text { IF } & \text { Initiation factor } \\ \text { kDa } & \text { Kilodalton } \\ \text { LB } & \text { Luria-Bertani medium } \\ \text { LSU } & \text { Large ribosomal subunit } \\ \text { M } & \text { Molar, mol/L } \\ \text { MDa } & \text { Megadalton } \\ \text { min } & \text { Minute } \\ \text { mL } & \text { Milliliter } \\ \text { mM } & \text { Millimolar } \\ \text { mRNA } & \text { Messenger RNA } \\ \text { nm } & \text { Nanometer } \\ \text { nM } & \text { Nanomolar } \\ \text { P site } & \text { PCR } \\ & \end{array}$




$\begin{array}{ll}\text { PDB } & \text { Protein data bank } \\ \text { pDNA } & \text { Plasmid DNA } \\ \text { PEP } & \text { Phosphoenolpyruvate } \\ \text { PK } & \text { Pyruvate kinase } \\ \text { pmol } & \text { Picomole } \\ \text { POST-complex } & \text { Post-translocation complex } \\ \text { PRE-complex } & \text { Pre-translocation complex } \\ \text { PTC } & \text { Peptidyl transferase center } \\ \text { RBS } & \text { Ribosomal binding site } \\ \text { RF } & \text { Release factor } \\ \text { RNA } & \text { Ribonucleic acid } \\ \text { rpm } & \text { Rotation per minute } \\ \text { R-protein } & \text { Ribosomal protein } \\ \text { RRF } & \text { Ribosome recycling factor } \\ \text { rRNA } & \text { Ribosomal RNA } \\ \text { RT } & \text { Room temperature } \\ \text { S } & \text { Svedberg unit } \\ \text { S } & \text { Second } \\ \text { SD } & \text { Standard deviaton } \\ \text { sm } & \text { Single molecule } \\ \text { SSU } & \text { Small subunit } \\ \text { TC } & \text { Ternary complex } \\ \text { TEMED } & \text { Tetramethylethylenediamine } \\ \text { tRNA } & \text { Transfer RNA } \\ \text { w/v } & \text { Weight/volume } \\ \text { w/w } & \text { Weight/weight } \\ \text { WT } & \text { Wild type } \\ \text { X-ray } & \text { X-ray crystallography } \\ \mu \text { M } & \text { Micromolar } \\ & \end{array}$




\section{Acknowledgments}

"My old grandmother always used to say, summer friends will melt away like summer snows, but winter friends are friends forever." - George R.R. Martin, A Feast for Crows

I am writing acknowledgments and that means my Ph.D. journey is coming to the end. It is time to thank the ones who were with me day by day and made these years truly wonderful. First and foremost, I thank Prof. Marina Rodnina for allowing me to join her team and for being an amazing example that inspires and encourages young minds to be brave and follow their aspirations and dreams. Your door is always open for us and no question is left unanswered. This drives us towards the right answers while unraveling scientific secrets, with no fear of failures. I am truly delighted and immensely proud to be learning and working under your supervision. I thank my TAC members, Prof. Holger Stark and Prof. Heike Krebber for sharing their knowledge and expertise, and Prof. Kai Tittmann, Prof. Wolfgang Wintermeyer and Prof. Ralf Ficner for contributing to the success of my graduate school. I would like to thank my direct supervisor, Ekaterina, who was always giving me work advice when needed and for teaching me valuable life lessons. Tamara, I thank you for the scientific guidance, personal support, and warmth. Frank, thank you so much for your help, patience, and encouragement. Sandra, mon petit chou, you were there for me when it hasn't been my day, my week, my month or even my year and I could not even dream of a friend like you. I hope our Russian-French connection will grow stronger over the years. Albena and Inessa, you supported me even being so far away, I would not make it until the end without your kind words. Dimitra, only because of you being by my side I stayed alive during the bureaucracy battles. My big international Göttingen family, my magic beans - Geoffrey (hope to see Saint Etienne playing in the UEL final one day), Monica (cheese and wine social club), Csaba\&Judit (it is a long story), Filippo (for teaching me basic Italian), Mark (old but good), Ole (best roomie in the world), Ilya (no pain no muscle gain), Leyla (bortsch of my soul), Davit (Armenian wisdom) and Sergey (shots!). You will be in my heart forever and I hope we stay friends for life, no matter where the Life sends us around the Globe. I thank all the people in the lab for making my days at work enjoyable despite the typical Göttingen 
weather - Ingo, Raffaella, Riccardo, Natalia, Betty, Nessa, Jan, Meline, Prajwal, Cristina, Olaf, Sandra, Moritz, Marija, Cathie, Manuela, Mario, Sara, Panos, Namit, Sakshi, SungHui, Tessa, Kärt, Evan, Xiaolin, Lena, Franzi, Anna, Mani, Christina, and Michael...My God, we are many. The happy faces on the hallway from the Stark Department should be also mentioned, especially you, my dear Dr. Henneberg. You bring me the biggest joy and courage to look forward to the future. Thank you for being with me when I am no princess, but a dragon myself.

Finally, I would like to thank my family, my Mama and Papa for listening, caring, loving, supporting and believing in me, no matter what. You made me who I am now and there are no words in this world to describe how much I love you. I dedicate my doctoral thesis to you and hope you can be proud of me.

And now, my watch is over. 



\section{Abstract}

During synthesis of a topoisomerase subunit encoded by the gene 60 of bacteriophage T4, the ribosome takes off from a certain codon, slides over a 50-nt non-coding segment of mRNA and resumes translation on a specific landing codon, which together is denoted as translational bypassing. The signals required for bypassing are programmed within the gene 60 mRNA and include the take-off and the landing codon, the stop codon adjacent to the take-off codon, several mRNA stem-loops and the nascent peptide that interacts with the exit tunnel of the ribosome. Gene 60 mRNA structures orchestrate ribosome movements during bypassing, but it is unclear which event initiates the take-off from the mRNA and what defines the directionality of sliding. We have investigated bypassing in a reconstituted in vitro translation system from $E$. coli and have probed the ribosome dynamics during bypassing using single-molecule FRET technique. We show that the nascent peptide interactions within the exit tunnel of the ribosome together with a short mRNA stem-loop formed in the A site induce a non-canonical hyper-rotated state of the ribosome during pausing and before the take-off. Elongation factor $G(E F-G)$ interacts with the pausing ribosome and facilitates pseudo-translocation, using the mRNA stemloop as an A-site tRNA mimic. This disrupts the codon-anticodon interaction in the P site and initiates the take-off. During forward sliding about two guanosine 5 '-triphosphate (GTP) molecules are hydrolyzed per nucleotide of the noncoding gap, which we suggest is important for efficient sliding and landing. Our data suggest that EF-G plays an important, previously unanticipated role in translational bypassing initiation.

Previous in-vivo data suggested that the ribosomal protein L9 may act as a regulator of bypassing, in particular in the context of polysomes. We show that polysome formation reduces the bypassing efficiency, whereas the deletion of the L9 does not increase bypassing in vitro. Our work reveals unforeseen details of the bypassing mechanism and regulation and opens up new perspectives for studies of recoding events. 
Introduction

\section{Introduction}

\subsection{Ribosome structure and function}

Translation of an messenger RNA (mRNA) into a polypeptide chain by the ribosome is one of the most fundamental biological processes. The ribosome is a complex ribonucleoprotein machine, which decodes the mRNA template and links aminoacids supplied by aminoacylated transfer RNAs (aa-tRNAs) into a polypeptide chain according to the sequence of codons on the mRNA. The ribosome consists of ribosomal RNAs (rRNAs) and ribosomal proteins (r-proteins), which are important for ribosome assembly and function. rRNAs form the catalytic sites of the ribosomal and are essential for the core functions of the ribosome such as peptide bond formation, mRNA and tRNA binding and GTPase activation of translation factors. R-proteins contribute to the ribosomal assembly, interact with the translational components, and stabilize the ribosomal structure by contacting several RNA elements simultaneously.

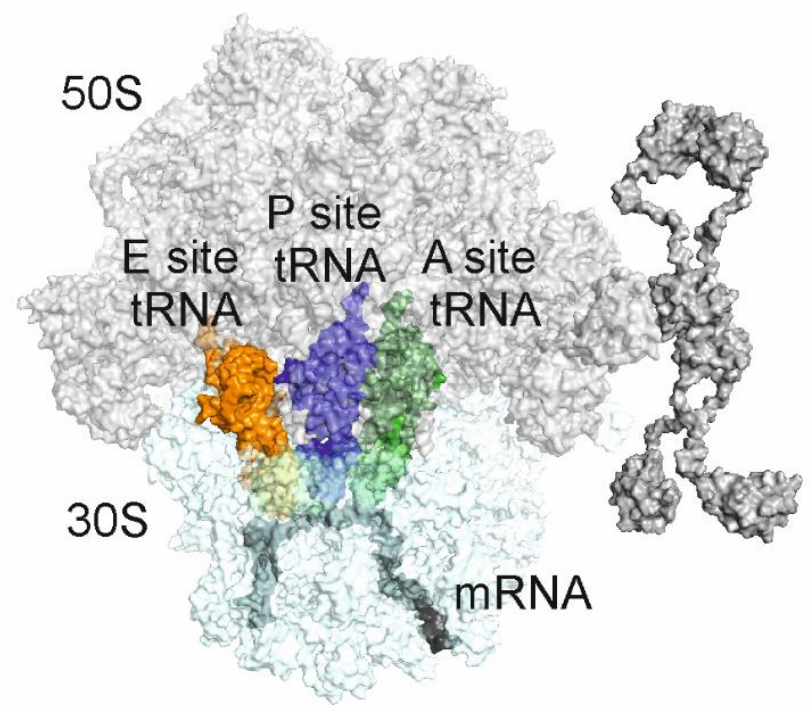

Figure 1. Structure of the bacterial ribosome (70S)

The 70S ribosome consists of two subunits: the large 50S subunit (light grey) and the small 30S subunit (light blue). The LSU contains the peptidyl transferase center and the peptide exit tunnel, the SSU houses the decoding center and the mRNA (dark grey) binding channel. Together, the two subunits form three tRNA binding sites: the $A$ site, the $P$ site and the $E$ site. The image was produced from structures with Protein Data Bank (PDB) accession numbers 3J4V, 3J52, 2QA4 and 3AIY (Bock et al., 2013; Kavran \& 
Steitz, 2007; Naganuma et al., 2010). The colour code for different components is used throughout this thesis.

Although ribosomes carry out the same function in all living organisms, they differ in size and complexity: the molecular weight of the bacterial ribosome is $2.3 \mathrm{MDa}$, while in higher eukaryotes it reaches 4.3 MDa. Historically, ribosomes are differentiated according to their sedimentation coefficient, the Svedberg (S) unit, and named the $70 S$ and $80 S$ for bacteria and eukaryotes, respectively. A golden era of understanding complex ribosomal architecture and the mechanisms of translation began when accurate atomic models of the bacterial ribosome became available due to advances in cryo-EM and X-ray crystallography (Schluenzen et al., 2000; Yusupov et al., 2001; Ramakrishnan, 2002; Steitz, 2008; Voorhees \& Ramakrishnan, 2013; Agirrezabala \& Frank, 2010). The ribosome forms three stable tRNA binding sites: the aminoacyl site (abbreviated the $A$ site) which binds the incoming aa-tRNA complementary to the mRNA codon, the peptidyl site (the $\mathrm{P}$ site) which harbors the peptidyl-tRNA with the growing polypeptide chain, and the exit site (the $E$ site) through which the deacylated tRNA is released.

The bacterial and eukaryotic ribosomes share a conserved ribonucleoprotein core, consisting of two unequal subunits both carrying out different roles during translation (Fig. 1) (Melnikov et al., 2012; Bieri et al., 2018). The small subunit (SSU), denoted as the $30 \mathrm{~S}$ in bacteria or $40 \mathrm{~S}$ in eukaryotes is composed of the $16 \mathrm{~S}$ rRNA and $21 \mathrm{r}$-proteins labeled S1 to S21 (18S rRNA and 33 r-proteins in eukaryotes). It encompasses 3 domains (the head, the body, and the platform) and houses the decoding center where aa-tRNA is recognized and selected according to the mRNA sequence. The SSU is also responsible for the mRNA engaging during translation, which binds at the cleft between the head and the body. R-proteins S3, S4 and S5 form the mRNA entry site, whereas the r-protein S12 is the only protein contributing to the decoding site. The large subunit (LSU), denoted as the 50S (60S in eukaryotes), is composed of the 23S and the 5S rRNA and $31 \mathrm{r}$-proteins labeled L1 to L31 (5S, 28S and 5.8S rRNA and 46 proteins in eukaryotes). Its main functional site is the peptidyl transferase center (PTC) located in the cleft on the intersubunit side, it is responsible for the peptidyl transfer reaction during elongation and release of the newly synthesized polypeptide chain during termination of translation. The 
Introduction

LSU also harbors the peptide exit tunnel, through which the growing nascent peptide chain leaves the ribosome (Ban et al., 2000). The r-protein L1 is involved in binding, movement, and release of deacylated-tRNA from the $E$ site by remodeling its structure (Cornish et al., 2009). The L7/12 stalk, which consists of ribosomal protein L11, the region of $23 \mathrm{~S}$ rRNA binding proteins $\mathrm{L} 11$ and L10, and a complex formed by L10 and multiple copies of $L 7 / 12$, assists the recruitment of translation factors (Diaconu et al., 2005). The process of protein synthesis requires multiple rearrangements of the LSU and the SSU relative to each other. There are several regions on the interface of ribosomal subunits (so-called intersubunit bridges) that stabilize the complete ribosome and ensure its dynamics at the same time. Some intersubunit bridges are supported by the metal ions like $\mathrm{K}^{+}$and $\mathrm{Mg}^{2+}$ and water molecules (Schuwirth et al., 2005; Jenner et al., 2010; Bieri et al., 2018; Rozov et al., 2019).

Besides cytoplasmic ribosomes, eukaryotes also have ribosomes in chloroplasts and/or mitochondria, the organelles originated from an $\alpha$-proteobacterial ancestor. The so-called mitoribosomes have a different RNA/protein ratio, which results in a lower sedimentation coefficient (29S for the SSU and 395 for the LSU forming a 55 S ribosome) and higher molecular weight (2.7 MDa) in comparison to the bacterial ribosome (Bieri et al., 2018). The protein synthesis in chloroplasts is catalyzed by a bacterial-type $70 \mathrm{~S}$ ribosome (designated as chlororibosome) composed of a 50S LSU and a 30 S SSU, suggesting that the essential functions like mRNA translation into a polypeptide chain have been conserved throughout evolution for dissimilar organisms (Tiller \& Bock, 2014).

\subsection{Translation cycle: an overview}

The translation cycle entails four major stages: initiation, elongation, termination, and ribosome recycling. To control polypeptide synthesis and ensure the optimal rate and fidelity of translation, multiple protein factors are recruited at different stages of the cycle (Rodnina \& Wintermeyer, 2009). These factors include initiation (IFs), elongation (EFs), release (RFs), and ribosome recycling (RRF) factors, some of which use guanosine 5'triphosphate (GTP) hydrolysis in facilitating protein synthesis on the ribosome (Rodnina, 2018). The translation process starts with the ribosome selecting the correct start codon 
within a given mRNA and ends when the ribosome arrives at the stop codon, which together defines an open reading frame (ORF). In bacteria, mRNAs can be polyscistronic, which means one mRNA molecule contains several encoded protein sequences. Having established a translational ORF on a given mRNA, the ribosome must maintain it during the course of translation. Efficient translation initiation relies on several specific elements of the mRNA. These include the nature of the codon used for initiation (mainly AUG, but in some cases also GUG or UUG), the mRNA secondary structure, the extended 5' untranslated region ( $5^{\prime}$ UTR), and the ribosome binding site (RBS) near the start codon (Rodnina, 2018). In many bacteria, the key element of the RBS is the so-called ShineDalgarno (SD) sequence located 8-10 nucleotides upstream of the start codon. The SD sequence pairs to the $16 \mathrm{~S}$ rRNA of the SSU, thereby facilitating mRNA recruitment to the ribosome. Initiation factor IF2 promotes initiation by recruiting the initiator aa-tRNA, fMettRNA ${ }^{\mathrm{fMet}}$. The initiation factors IF1 and IF3 both ensure the correct initiator tRNA assembly in the P site of the SSU on the mRNA. After the initiator tRNA anticodon pairs with the AUG mRNA start codon, the ribosome complex matures into a 305 initiation complex (Milón \& Rodnina, 2012). Joining the LSU triggers GTP hydrolysis by IF2, dissociation of all initiation factors, and formation of the $70 S$ initiation complex ready for the translational elongation phase. The most common and best-studied mRNAs contain a SD sequence (Milón \& Rodnina, 2012) but prokaryotes also have mRNAs lacking SD sequence (Chang et al., 2006) and even leaderless mRNAs without a 5'UTR (Zheng et al., 2011) whose initiation mechanism is yet not fully understood. Eukaryotes have evolved a sophisticated mechanism of translation initiation, although some features are conserved between the kingdoms. The mRNA path in bacterial and mammalian ribosomes share certain structural similarities and some rRNA contacts with the P-site tRNA were found to be functionally conserved (Dong et al., 2008; Pisarev et al., 2008). In contrast to prokaryotes, eukaryotic initiation requires an assembly of multiple initiation factors and involves a scanning mechanism. The SSU loaded with initiator aa-tRNA in complex with initiation factors finds a start codon associated with a consensus mRNA sequence, a so-called Kozak sequence (Kozak, 1987). Most eukaryotic mRNAs contain a 5' 7-methyl guanosine cap and a 3' poly-adenosine tail; these features are necessary for conventional mRNA recruitment and regulation of translation initiation (Sonenberg \& Hinnebusch, 2009; Aitken 
Introduction

\& Lorsch, 2012). Some eukaryotic mRNAs employ alternative cap-independent mechanisms of initiation based on internal ribosome entry sites (IRES). During initiation on an mRNA with an IRES, the scanning step is omitted and the ribosomes are positioned directly on the start codon, guided by highly structured mRNA signals. Recent studies also suggested the so-called concept of cap-independent translation enhancers (CITEs). In this case, the recruited mRNA also lacks the 7-methyl guanosine cap but is then inspected by the scanning machinery in the same way as under cap-dependent initiation (Shatsky et al., 2018).

The elongation phase starts when the ribosome reads the second codon of the ORF and ends when it reaches the stop codon. Elongation includes three main steps which are repetitive cycles of decoding, peptide bond formation, and translocation. The decoding process relies on the complementary Watson-Crick ( $G-C$ and $A-T$ ) base matching between the codon of the mRNA in the A site of the SSU and the anticodon of the incoming aa-tRNA. Accurate decoding depends on the ability of the ribosome to distinguish between matching (cognate) and non-matching (near-cognate and noncognate) tRNAs. The tRNAs which are able to form three stable base pairs with the codon triplet according to Watson-Crick rules are defined as cognate tRNAs. In contrast, tRNAs that do not meet these requirements are commonly referred to as near- and non-cognate tRNAs (one and at least two mismatches, respectively). The pairing of the third base is not strict and allows a wobble pair formation, which is essential for the translation of the genetic code (Crick, 1966). Elongation factor Tu (EF-Tu) in its GTP-bound conformation (EF-Tu-GTP) forms a high-affinity complex with aa-tRNA (ternary complex) and delivers it to the A site, which is followed by peptide bond formation and nascent peptide chain extension. Following GTP hydrolysis EF-Tu dissociates from the ribosome in an inactive GDP-bound conformation. Elongation factor Ts (EF-Ts) serves as a guanine nucleotide exchange factor for EF-Tu, promoting the GDP release from the latter and allowing the formation of new active EF-Tu-GTP. After successful peptide bond formation, elongation factor $G$ (EF-G) induces tRNA-mRNA complex translocation at the cost of GTP hydrolysis, and the ribosome moves by one codon along the mRNA to enter the next round of elongation (Rodnina et al., 1997). The mechanism of translation elongation is 
well conserved between eukaryotes and bacteria, albeit decoding in eukaryotes appears to be slower (Rodnina \& Wintermeyer, 2009). As in prokaryotes, eEF1A-GTP (the analog of EF-Tu) delivers aminoacyl-tRNA to the vacant $A$ site of the ribosome, whereas eEF1B (the analog of EF-Ts) is responsible for the GDP-to-GTP exchange. eEF2 (the analog of EF-G) promotes translocation of the tRNA-mRNA complex (Rodnina \& Wintermeyer, 2009; Dever \& Green, 2012).

When the ribosome encounters a stop codon in the ORF, the translation process is terminated. In bacteria, the three stop codons are recognized by two proteins with overlapping specificities: release factor 1 (RF1) recognizes the stop codons UAG and UAA, whereas release factor 2 (RF2) recognizes UGA and UAA (Scolnick et al., 1968). GTPase release factor 3 (RF3) is not required for stop codon recognition and peptidyl tRNA hydrolysis but facilitates RF1 and RF2 recycling (Zaher \& Green, 2011; Adio et al., 2018). Upon stop codon recognition the synthesized nascent polypeptide is detached from the peptidyl-tRNA as a result of a hydrolysis reaction catalyzed by the PTC with the help of a highly conserved Gly-Gly-Gln (GGQ) motif in RF1/RF2 (Scarlett et al., 2003). Although RF1 and RF2 promote peptide release by a similar mechanism, structures of these factors bound to termination complexes revealed differences in their interaction with the LSU, in particular with the L11 region (Korostelev et al., 2008; Laurberg et al., 2008 Korostelev et al., 2010). Binding of RF3 in the GTP-bound conformation and peptide chain release causes RF1 dissociation, which is followed by GTP hydrolysis and consequent dissociation of RF3 in the GDP-bound conformation. RF2 does not need an auxiliary factor and can dissociate spontaneously (Adio et al., 2018). After termination, the ribosomes still retain deacylated tRNA and mRNA, which both have to be released in order to start the new initiation round. Disassembly of the post-termination complex in bacteria is catalyzed by EF-G and RRF (ribosome recycling factor). RRF stabilizes the ribosome, whereas GTP hydrolysis by EF-G promotes conformational changes of RRF on the ribosome, leading to subunit splitting and thereby to post-termination complex dissociation (Gao et al., 2005). The dissociation of tRNA from the SSU is promoted by IF3, while the mRNA is exchanged spontaneously (Peske et al., 2005). 
Introduction

Termination in eukaryotes is catalyzed by two protein factors, eRF1 (class I) and eRF3 (class II), which were shown to collaborate in the process (Alkalaeva et al., 2006). eRF1 recognizes all stop codons and performs peptidyl-tRNA hydrolysis using the universally conserved GGQ motif. eRF3 is a GTPase and accelerates peptide release and increases termination efficiency at stop codons forming a complex with eRF1 (Frolova et al., 1996; Frolova et al., 1999). Recycling of the ribosome in eukaryotes is coupled to termination and differs from prokaryotes as it is mediated by several initiation factors and involves the recruitment of auxiliary protein factors like ligatin (elF2D) and density-regulated protein (DENR) (Hellen, 2018). However, in some cases instead of full disassembly of the ribosomal complex during termination, only partial dissociation occurs, whereby allowing translation on the same mRNA. This type of events is loosely termed as reinitiation. Such partial recycling might also take place at the stop codon of an mRNA containing a single ORF, allowing for scanning along the $3^{\prime}$ UTR and facilitating the transfer of the LSU to the $5^{\prime}$ UTR and a subsequent round of translation of the same ORF (Dever \& Green, 2012).

\subsection{Elongation cycle and ribosome dynamics}

The elongation cycle involves repetitive cycles of mRNA decoding, tRNA accommodation, peptide bond formation and tRNA-mRNA translocation, which results in a forward movement of the ribosome along the mRNA as the nascent chain is synthesized. The SSU plays a critical role during decoding, it accurately selects tRNAs delivered by EF-Tu in accordance with the mRNA codon triplet placed in the decoding center. The shape and activity of the decoding center are majorly defined by $16 S$ RNA, whose secondary structure contains multiple double helices stabilized by r-proteins. The functionally important area of the decoding center at the interface with the LSU is RNArich and almost protein-free, except for r-protein S12 (Wimberly et al., 2000). Structural and biochemical studies showed, that during the decoding process r-protein $\mathrm{S} 12$ makes direct contacts with the codon-anticodon helix in the $A$ site and serves as a modulator of decoding accuracy. Moreover, the absence of $\mathrm{S} 12$ contributed to the translation activity in the EF-Tu and EF-G free system (Ogle et al., 2001; Cukras et al., 2003; Demirci et al., 2013). 
Successful codon recognition causes conformational changes of the ribosome, which stabilizes tRNA binding and induces the GTPase activity of EF-Tu (Rodnina et al., 2002). The GTP hydrolysis step is crucial for the optimization of both speed and accuracy of the translation elongation phase. The rate of GTP hydrolysis differs for the cognate, nearcognate and non-cognate ternary complexes, which allows the discrimination against non-matching tRNAs (Rodnina et al., 1995; Wohlgemuth et al., 2010). EF-Tu in a GDPbound conformation (EF-Tu-GDP) has low affinity for aa-tRNA and dissociates from the ribosome, whereas the aa-tRNA moves into the LSU A site, accommodates in the PTC and takes part in the next translation elongation step, namely peptide bond formation (Liu et al., 2015; Maracci \& Rodnina, 2016). The mechanism of peptide bond formation is presumed to be highly conserved in all kingdoms of life. A new peptide bond is formed in the PTC located on the LSU as a result of a nucleophilic attack of an aminoacyl-tRNA in the $A$ site on ester carbon of the peptidyl-tRNA in the $P$ site (Beringer et al., 2005). Notably, the peptidyl transfer reaction is catalyzed solely by the rRNAs of the PTC, which makes the ribosome the only known natural ribozyme with polymerase activity (Rodnina, 2018).

After the formation of a peptide bond, the peptidyl-tRNA moves from the A site to the $P$ site and the deacylated tRNA from the $P$ to the $E$ site and this translocation process is coupled to the movement of the mRNA by one codon. This movement is facilitated by EF$G$ at the cost of GTP hydrolysis. In the absence of EF-G spontaneous tRNA-mRNA reverse movement was shown to be also possible, indicating that this factor defines the directionality of tRNA movement during protein synthesis (Konevega et al., 2007; Adio et al., 2015). Initially, after a peptidyl transfer reaction the A- and P-site tRNAs reside in their classical state (denoted as $\mathrm{A} / \mathrm{A}$ and $\mathrm{P} / \mathrm{P}$ configuration) and together with ribosome and mRNA form a pre-translocation (PRE) complex. During translocation, the A- and P-site tRNAs are first moved to a hybrid state designated as $A / P$ and $P / E$ configuration, where the anticodon stem-loops of the tRNAs stay in the A and P sites on the SSU, whereas their acceptor ends are moved to the $P$ and $E$ sites on the LSU, respectively. The formation of $A / P$ and $P / E$ configuration is spontaneous and driven by the thermal motion of the ribosome. Notably, the PRE complex is extremely dynamic and fluctuates between 
Introduction

classical and hybrid states both populated to a comparable extent, but the hybrid state is enhanced and stabilized by EF-G binding, which is necessary for the further tRNA movement (Cornish et al., 2008; Dunkle et al., 2011; Holtkamp et al., 2014; Adio et al., 2015; Sharma et al., 2016). Binding of EF-G to the ribosome induces GTP hydrolysis. This facilitates the interactions of EF-G with the ribosome and remodels the energy landscape of translocation, allowing rapid and synchronous tRNA-mRNA movement on both SSU and LSU into the POST state. As a result, a post-translocational (POST) complex is formed, in which the peptidyl-tRNA resides in classical P/P configuration. The translocation cycle ends with the now-deacylated E-site tRNA leaving the ribosome, the $P$ site holding the peptidyl-tRNA, and the vacant $A$ site ready for the next ternary complex accommodation.

One of the important results emerging from studies on the prokaryotic translation is the view of the ribosome as a dynamic structure (Rodnina, 2018). During the course of translation, ribosomal subunits move relative to each other promoting the directionality of protein biosynthesis. The translocation step requires not only movements of the two tRNA molecules together with the mRNA, but also coordinated movements of the ribosomal subunits relative to each other. The tRNAs movement from the classical to hybrid states upon peptide bond formation is accompanied by rotation of the SSU relative to the LSU in a counter-clockwise (CCW) direction around an axis running normal to the plane separating the subunits (Frank \& Agrawal, 2000; Ermolenko et al., 2007) (Fig. 2). Thus, during the formation of the PRE state, the ribosomal conformation is changed from the classical non-rotated to the hybrid rotated state, and the head domain of the SSU swivels relative to the body domain forward in the direction of tRNA movement (Guo \& Noller, 2012). In the PRE state, the SSU and the LSU spontaneously fluctuate between nonrotated and rotated states, binding of EF-G-GTP stabilizes the latter. GTP hydrolysis causes conformational rearrangements of EF-G that induce the SSU body to move clockwise $(\mathrm{CW})$ from the rotated to the non-rotated state; in contrast, the head domain of the SSU remains in a swiveled state. Pi release from EF-G and synchronous tRNA movement into the classical P/P and E/E states successively returns both the SSU head 
and the body to the initial non-rotated state and leads to POST state formation (Belardinelli \& Rodnina, 2017).

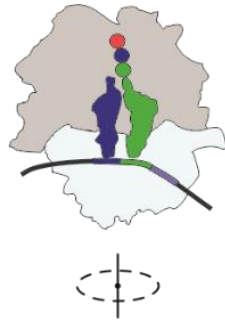

non-rotated, $0^{\circ}$

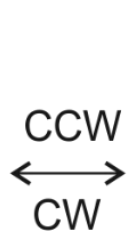

$\mathrm{CW}$
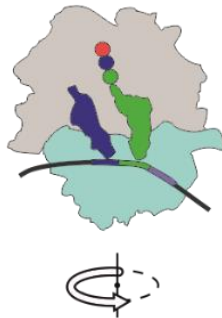

rotated, $7-8^{\circ}$
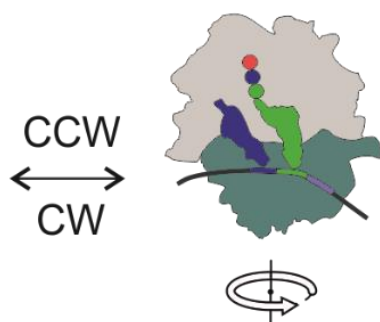

hyper-rotated, $12-18^{\circ}$

Figure 2. Conformational dynamics of the ribosome

Ribosomal subunit rotation from the non-rotated to the rotated state involves the counterclockwise rotation of the SSU with respect to the LSU (light blue to turquoise). The subunit rotation from the non-rotated to the rotated state is a spontaneous and reversible process. The further SSU rotation leads to hyper-rotated state formation (turquoise to see green) and requires additional external conditions. Arrows indicate the direction of rotation.

Some additional factors like structured mRNA can drive the ribosomal subunits into a non-canonical hyper-rotated state, which represents further SSU rotation in the CCW direction (Fig. 2). Ribosomes paused in such hyper-rotated state were detected in the presence of frameshift inducing dnaX hairpin (Qin et al., 2014; Chen at al., 2014).

The rapid ribosome progression along the mRNA is crucial for correct polypeptide synthesis during translation. Ribosome pausing during translocation may lead to recoding, which causes the alternative readout of the genetic information (recoding is discussed in detail in the following section). Besides its role in promoting fast translocation, EF-G has recently been shown to help the ribosome to maintain the established ORF by guiding the A-site tRNA and facilitating rearrangements of the ribosome, thereby restoring the ribosome control over the codon-anticodon interaction and ensuring the correct mRNA decoding (Peng et al., 2019).

\subsection{EF-G}

Translocation of tRNA and mRNA on the ribosome is promoted by EF-G, which is an essential translational factor and a universally conserved guanosine triphosphatase 
Introduction

(GTPase). EF-G uses the energy of GTP hydrolysis to promote fast tRNA-mRNA translocation and can rapidly exchange GDP to GTP without an auxiliary nucleotide exchange factor (Pulk \& Cate, 2013; Bourne et al., 1991; Wilden et al., 2006). EF-G performs GTP hydrolysis only when bound to the ribosome; its binding occurs through the ribosomal protein $L 7 / L 12$. The GTPase activity of EF-G is activated after the ribosome rearranges its catalytic site into the optimal conformation (Rodnina et al., 2019).

EF-G consists of about 700 amino acids arranged into five domains, denoted as domain I to domain $\mathrm{V}$, which undergo large rearrangements during the process of translocation. Domain I (or the G domain) binds and hydrolyzes GTP and promotes EF$G$ binding to the ribosome through the interactions with the LSU. In complex with the PRE ribosome domain II of EF-G interacts with the 16S RNA of the SSU (Brilot et al., 2013; Lin et al., 2015). Domains II, III and V are positioned adjacent to the r-protein L11, while domain IV is extended towards the SSU A site. It was unclear how domain IV avoids collision with the A-site tRNA upon binding to the PRE ribosomal complex until recent structural studies revealed a compact conformation of EF-G in solution (Fig. 3A-D). 
A

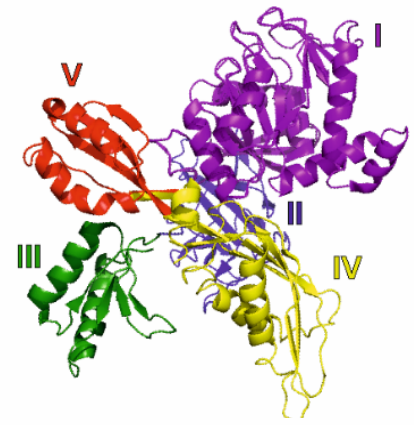

B

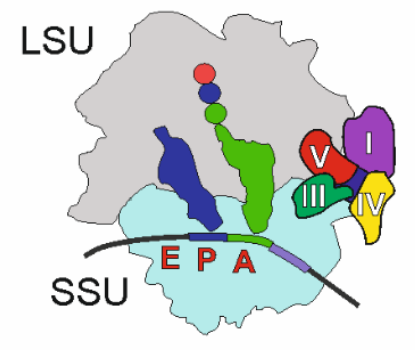

PRE
C

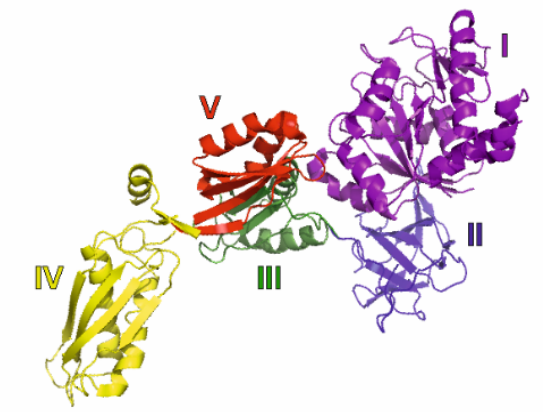

D

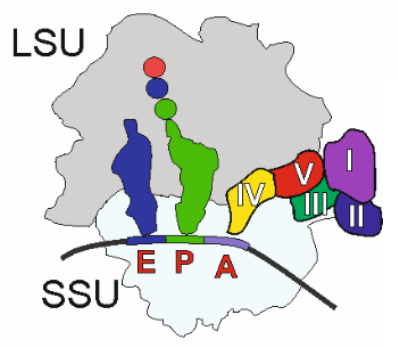

POST

Figure 3. Different EF-G conformations during translocation

A schematic overview of the conformational changes of the ribosome and EF-G during translocation in PRE and POST complexes. Binding of EF-G-GTP in the compact form (A) to pre-translocation (PRE) complex (B) stabilizes the rotated state (turquoise). EF-G changes its conformation to the elongated form (C), thereby inducing synchronous tRNA-mRNA movement followed by the motion of the SSU towards the original non-rotated state (light blue), corresponding to POST complex (D) formation. The image was produced from structures with Protein Data Bank (PDB) accessions 4WPO and 4WQY (Lin et al., 2015)

EF-G consists of two super-domains formed by domains I-II and domains III-V, connected through a flexible linker. This makes EF-G highly dynamic and allows it to fluctuate between two distinct conformations (Lin et al., 2015). Apart from the extended conformation, EF-G was shown to adopt a compact conformation, where domains III-IV$\mathrm{V}$ are rearranged and positioned closer to domains I-II, thereby avoiding a steric clash with the anticodon stem-loop of the A-site tRNA during binding to the ribosome (Salsi et al., 2015; Lin et al., 2015). EF-G hydrolyzes GTP immediately after binding to the ribosome, driving domain rearrangements, which in turn disrupts interactions of the tRNA with the ribosome and changes the conformation of the SSU. EF-G action thus prevents backward tRNA movement and favors the POST ribosomal complex formation. 
Introduction

Therefore, the main function of EF-G is to convert chemical energy from GTP hydrolysis into directional movement of the ribosome in a manner similar to the power stroke of molecular motors (Savelsbergh et al., 2003; Chen et al., 2016; Rodnina et al., 1997; Holtkamp et al., 2014; Salsi et al., 2015).

\subsection{Major determinants of the translation rate}

Each step of the elongation phase can alter the translational efficiency in multiple ways. Changes in local translational velocity may alter the fidelity of translation (Rodnina, 2016) and protein folding (Trovato \& O'Brien, 2017), and, as a result, affect the quality of the synthesized protein, leading to the deterioration of cell viability. The speed of translation also directly affects ribosomal turnover and the ribosome density on the mRNA, which highly correlates with protein abundance in the living cell ( $\mathrm{Li}$ et al., 2014; Rodnina, 2016). The coding region of the mRNA may contain special regulatory signals, which together with some auxiliary factors govern the ribosomal acceleration or pausing during translation and affect the overall protein synthesis time. These factors include: 1 ) codon usage together with the tRNA pool composition, which modulates the rates of cognate aa-tRNA delivery and decoding (Komar et al., 1999; Hussmann et al., 2015; Chevance et al., 2014); 2) amino acid composition of the encoded peptide chain (Charneski \& Hurst, 2013; Dao Duc \& Song, 2018); and 3) the presence of significant mRNA secondary structures (discussed in detail in the following section) (Rodnina, 2016).

\subsubsection{Translation rate modulated by the nascent polypeptide}

Codon usage and tRNA abundance were considered to be the major rate-limiting factors of translation until the ribosome profiling technique was developed and optimized (Ingolia et al., 2009) which allowed a re-evaluation of the previous assignment of the codon usage being the primary elongation rate determinant. Although the tRNA abundance level and the codon usage do influence the translation rate, define the pausing patterns and support the proper protein folding (Komar, 2009; Komar, 2016), it does not comprehensively explain the observed translational rate variations under normal conditions. According to observations in ribosome profiling datasets, the sequence of a nascent peptide bound to the ribosome also plays a role in modulating ribosomal 
progression along the mRNA (Charneski \& Hurst, 2013; Dao Duc \& Song, 2018). More specifically, peptides containing stretches of positively charged residues downregulate translation and contribute to increased ribosomal density on the mRNA (Charneski \& Hurst, 2013). Moreover, the insertion of a polylysine sequence causes translational repression and a later degradation of the produced peptide (Ito-Harashima et al., 2007). The impeding effect is not related to the residue's shape or size, but specifically to its electric charge, and is mediated by the negatively charged LSU exit tunnel (Lu \& Deutsch, 2008). Electrostatic interactions within the ribosome tunnel inhibit the movement of the oppositely charged chain, and the hydropathy of a nascent peptide is suggested to explain up to $80 \%$ of the observed translational rate variations (Dao Duc \& Song, 2018). However, unambiguous interpretation of ribosomal profiling data is difficult due to the different analysis methods and current lack of high-throughput experimental measurements of translation rates in the cell. This leads to contradictory results regarding the impact of codon usage, tRNA concentrations and charged nascent chain residues on the rate of translation during the elongation phase (Dana \& Tuller, 2014; Weinberg et al., 2016; Sharma et al., 2019).

\subsubsection{Peptide-mediated stalling}

Peptide bond formation at the PTC requires accurate placement of the aa-tRNA substrates and proceeds through a nucleophilic attack of the a-amino group in the A site onto the carbonyl carbon of peptidyl-tRNA in the $\mathrm{P}$ site. The rate of this chemical reaction is different for each amino acid, but it has little effect on the overall translation rate. The accommodation of a tRNA in the A site limits the rate of peptide bond formation, therefore, the peptidyl transfer reaction is uniform for most aa-tRNAs, in spite of their intrinsic chemical reactivities (Bieling et al., 2006; Wohlgemuth et al., 2008). There are some exceptions: specific amino acids or amino acid combinations disfavor peptide bond formation and can slow translation down or even cause translational arrest. For example, proline is the only amino acid with a distinctive cyclic structure, which leads to unfavorable Pro-tRNA ${ }^{\text {Pro }}$ positioning in the PTC and altered trajectory for the nucleophilic attack during the peptidyl transfer reaction. This affects the rate of peptide bond formation between proline and other amino acids, thus making Pro an exceptionally poor P-site substrate 
Introduction

(Wohlgemuth et al., 2008; Doerfel et al., 2015). Polyproline sequences cause translational stalling and require a specialized translation factor (elongation factor P, EF-P) to alleviate the latter (Ude et al., 2013; Doerfel et al., 2013). Proline is present in many arrest peptide sequences, such as secretion monitor (SecM) (Nakatogawa \& Ito, 2002) and tryptophanase operon leader (TnaC) peptides (Gong \& Yanofsky, 2002), yet there are some examples of proline independent stalling, such as membrane protein insertion and folding monitor (MifM) (Chiba et al., 2009) and methyltransferase leader peptide (ErmCL/ErmBL) sequences (Gupta et al., 2016). All these sequences share one common feature: upon being synthesized they interact with the exit tunnel wall. The nascent peptide chain can fold into compacted secondary structures or even small $\alpha$-helical domains within the ribosomal exit tunnel (Woolhead et al., 2004; Lu \& Deutsch, 2005; Thommen et al., 2017). The exit tunnel spans around $100 \AA$ and can accommodate more than 30 amino acids depending on the specific polypeptide sequence. The exit tunnel comprises three folding zones: the upper region associated with PTC, the constriction region (with r-proteins L4 and L22 reducing the tunnel's width) and the lower region connected to the exit (Ban et al., 2000; Nissen et al., 2000). The initial notion that the tunnel wall is non-interactive was disproven by cryo-EM studies showing the distinct contacts of the nascent peptide segments with the ribosomal tunnel wall (Seidelt et al., 2009; Agirrezabala et al., 2017). Moreover, being mostly negative in electrostatic charge, it interacts with the nascent peptide side chains (Lu \& Deutsch, 2008), thereby causing nascent peptide chain stabilization, misalignment of reactive groups at the PTC and inactivation of the latter (Lu et al., 2011; Ito \& Chiba, 2013; Wilson et al., 2016; Agirrezabala et al., 2017).

\subsubsection{Connection between the mRNA secondary structure and translation rate}

Natural mRNAs can form secondary/tertiary structures, which the ribosome unfolds in order to continue translation. Strand separation activity is inherent to the ribosome: $r$ proteins S3, S4, and S5 form the ring-shaped mRNA entry site, allowing the ribosome to separate the mRNA strands with no auxiliary helicases involved (Takyar et al., 2005). It was suggested that the ribosome uses two active mechanisms to unwind mRNA. First, it destabilizes the mRNA helical junction at the entry site by biasing its thermal fluctuations 
towards the open state. Second, ribosomal conformational changes associated with translocation generate a force, therefore the ribosome mechanically pulls apart the mRNA single strands (Qu et al., 2011). However, the initial notion that mRNA unwinding during normal translational course causes ribosomal pausing was questioned by the results suggesting that ordinary mRNA secondary structures can not elucidate the observed pausing (Lu \& Deutsch, 2008). Nonetheless, some thermodynamically stable mRNA elements (like pseudoknots or stem-loops) are known to disturb ribosome dynamics during translocation and to slow down ribosomal movement (Chen et al., 2013). For instance, some mRNA sequences with stem-loops and pseudoknots (for example, from dnaX gene in E. coli) cause ribosome stalling in the long rotated state, which affects the reading frame maintenance and leads to programmed ribosome frameshifting, a special case of recoding (Gurvich et al., 2003; Brierley \& Dos Ramos, 2006; Caliskan et al., 2014; Qin et al., 2014; Chen at al., 2014).

\subsection{Translational recoding}

Translational errors can arise due to incorrect charging of a tRNA or misreading of an mRNA codon by an incorrect aminoacyl-tRNA on the ribosome. Generally, the fidelity of decoding is extremely high with a rate of spontaneous error frequencies in the range of $10^{-7}-10^{-4}$ depending on the type of error and its position in the newly synthesized protein (Garofalo et al., 2019). However, in some cases, the ribosome can be reprogrammed to read an mRNA in alternative ways (Rodnina et al., 2019). A number of genes, from viruses to higher eukaryotes, have evolved to use alternative translation events in order to regulate their own expression. To distinguish these alternative translation events from normal decoding process and spontaneous missense errors, they are called recoding. There are three major types of recoding events: stop codon readthrough, programmed ribosomal frameshifting and translational bypassing (Baranov et al., 2002) (Fig. 4). 
Introduction

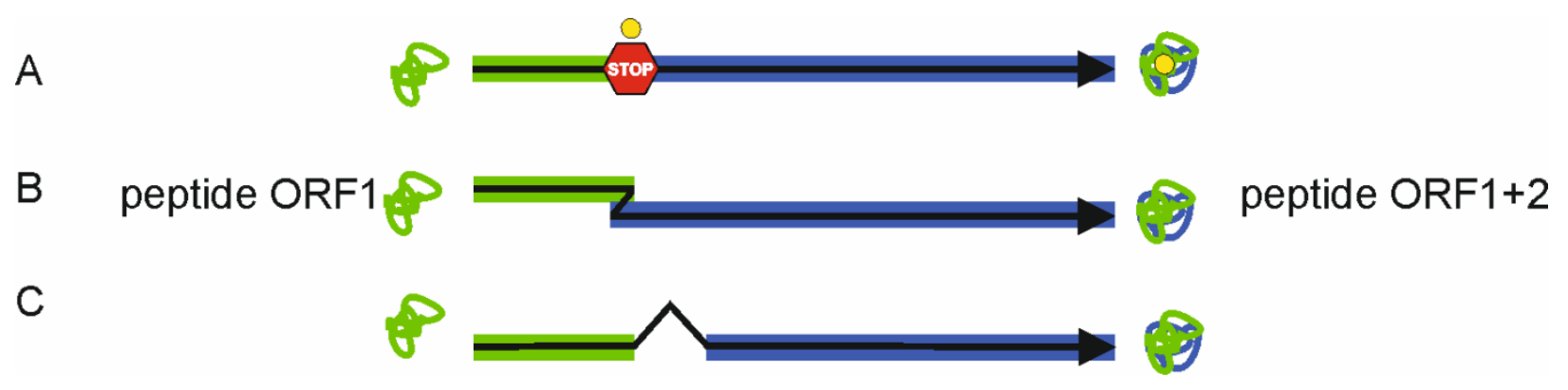

Figure 4. Programmed recoding events

Readthrough $(A),-1$ frameshifting $(B)$ and translational bypassing $(C)$. During translation the ribosome can be reprogrammed to read the mRNA in alternative ways. Black arrows indicate the direction of ribosome movement during recoding, green and dark blue colors represent ORF1 and ORF2, respectively. Polypeptide chains synthesized during recoding are drawn on the right. In readthrough, the yellow circle represents an amino acid incorporated at the stop codon (STOP) (Rodnina et al., 2019).

\subsubsection{Stop codon readthrough}

During translational termination, stop codons UAA, UAG and UGA are recognized by release factors through the sequence- and shape- specific codon recognition. In rare cases, a stop codon in a specific mRNA context can be read by an alternative cognate aa-tRNA, resulting in stop codon readthrough and incorporation of a non-canonical (pyrrolysine or selenocysteine) amino acid or by a near-cognate tRNA (Baranov et al., 2002; Rodnina et al., 2019). The incorporation of non-canonical amino acids expands the capacity of the genetic code. Notably, Sec and Pyl insertions were shown to follow different decoding strategies. Sec specification by a UGA stop codon is promoted by the presence of a stem-loop structure in the mRNA, the selenocysteine insertion sequence (SECIS) element, whereas Pyl insertion associated with a UAG codon can effectively compete with translation termination without a special mRNA guiding sequence (Zhang et al., 2005). Readthrough by a near-cognate tRNA, such as Cys, Trp, Tyr, GIn tRNA is also possible and leads to ORF expansion and production of two protein isoforms from the same mRNA (Blanchet et al., 2014). The minimal mRNA sequence motif that modulates spontaneous readthrough includes the identity of the stop codon and its upstream and downstream context. Utilization of the UGA stop codon and two adenines 
at positions -1 and -2 promotes readthrough, so does the presence of cytidine at position +4 and more distal stimulatory mRNA elements downstream the stop codon (Li \& Rice, 1993; Tork et al., 2004; Williams et al., 2004). Translational readthrough is widely used by virusesnbut was also found in genes from different species, including bacteria and higher eukaryotes (Skuzeski et al., 1991; Firth et al., 2011; Loughran et al., 2014)

\subsubsection{Programmed ribosomal frameshifting}

During translocation, interactions of the ribosome with the codon-anticodon complex should disengage to allow the forward movement. In the case of special "slippery" mRNA sequences, this loss of stabilizing interactions may result in re-pairing of the tRNAs with a codon in the -1 or +1 frame, leading to translation of the alternative ORF and the formation of an altered polypeptide sequence. Notably, ribosomal frameshifting can also be spontaneous but then results in a non-functional polypeptide production, whereas programmed translational frameshifting (PRF) leads to a synthesis of an altered, but yet functional protein. PRF can proceed both in forward (+PRF) or backward (-PRF) direction (Belcourt \& Farabaugh, 1990; Korniy et al., 2019). The most studied example of PRF is 1PRF, which is predominantly facilitated by a heptanucleotide slippery site sequence (Licznar et al., 2003) and an mRNA secondary structure element at a precisely defined distance from the latter (Brierley et al., 1989). Recent studies show that -1PRF follows one of two main possible pathways, the choice of which is determined by the cellular conditions rather than by the frameshifting sequences (Korniy et al., 2019). One route is exploited under conditions when the tRNAs necessary for the decoding of the slippery sequence are abundant. In this case, the presence of the mRNA stimulatory element is required, because it stalls the ribosome on the slippery sequence. As a result, the $A$ - and P-site tRNAs can re-pair in the alternative ORF (the most common is in -1 frame) while moving through the ribosome at the late stage of translocation (Kima et al., 2014; Caliskan et al., 2014; Yan et al., 2015). The other -1PRF pathway is induced by aa-tRNA limitation, e.g. during starvation, and is often called "hungry" frameshifting; its efficiency is independent of the downstream mRNA stimulators. Under reduced tRNA supply the ribosome spends more time waiting for a cognate tRNA to enter the vacant $A$ site, which opens a time window for the P-site tRNA to slip (Gallant \& Lindsley, 1992; Caliskan et al., 
Introduction

2017; Korniy et al., 2019). Recent studies have revealed that the propensity of $-1 P R F$ is defined mainly by the thermodynamic stability of potential mRNA-tRNA base pairing in the 0 - and -1 -frames, meaning that efficiency of $-1 P R F$ is determined only by the freeenergy difference between the alternative ORFs (Bock et al., 2019). -1PRF was shown to be also facilitated by binding of small non-coding RNA molecules or proteins to the sequence following the slippery site (Matsumoto et al., 2018; Yao et al., 2013; Wang et al., 2019). PRF enriches the coding capacity of a genome and occurs in all forms of life, from simple bacterial viruses to higher mammals (Manktelow et al., 2005; Belew et al., 2014).

\subsubsection{Translational bypassing}

Translational bypassing is an unusual recoding mechanism employed by the ribosome to synthesize a single peptide product from two discontinuous mRNA open reading frames separated by a non-coding gap. Most of our knowledge about translational bypassing comes from studies of bacteriophage T4 gene 60 (Huang et al., 1988), however, other examples of bypassing have recently been found in the mitochondrial genome of the yeast Magnusiomycetes (Lang et al., 2014; Nosek et al., 2015). Gene 60 has two open reading frames ORF1 and ORF2 separated by 50 nucleotides ( $\mathrm{nt}$ ). These two open reading frames together encode a single polypeptide chain, the DNA topoisomerase small subunit. The gap appears to represent a mobile genetic element inserted into the gene $60 \mathrm{mRNA}$ to inhibit cleavage by homing endonuclease MobA (Bonocora et al., 2011). Translation of ORF1 proceeds to the GGA codon 46, coding for glycine (the take-off site) (Fig. 1.7A). The subsequent codon is a UAG stop codon, and normally translation would be terminated here, but instead of terminating protein synthesis, the ribosome slides over a $50 \mathrm{nt}$-long non-coding gap, lands at a matching GGA codon (landing site) and resumes translation until the end of ORF2 (Maldonado \& Herr, 1998; Weiss et al., 1990; Herr et al., 2000). Remarkably, gene 60 mRNA is highly structured (Todd \& Walter, 2013) and contains all the signals required for bypassing regulation, meaning that no auxiliary factors apart from standard elongation factors are needed to recapitulate bypassing in vitro (Samatova et al., 2014). These signals include a stem-loop (SL) containing the take-off codon, the UAG stop codon subsequent to the 
take-off site, a sequence of the nascent peptide chain encoded upstream of the take-off site and two SL elements upstream the take-off site (5'SL) and downstream (3'SL) of the landing site (Fig.5A) (Herr et al., 2004; Weiss et al., 1990; Samatova et al., 2014) The matching GGA take-off and landing codons are crucial, as well as the identity of peptidyltRNA ${ }^{\text {Gly }}$ GGA in the $P$ site (Bucklin et al., 2005). According to chemical and enzymatic probing, the non-coding gap is largely unfolded and forms a module that is structurally independent of the two ORFs (Todd \& Walter, 2013).

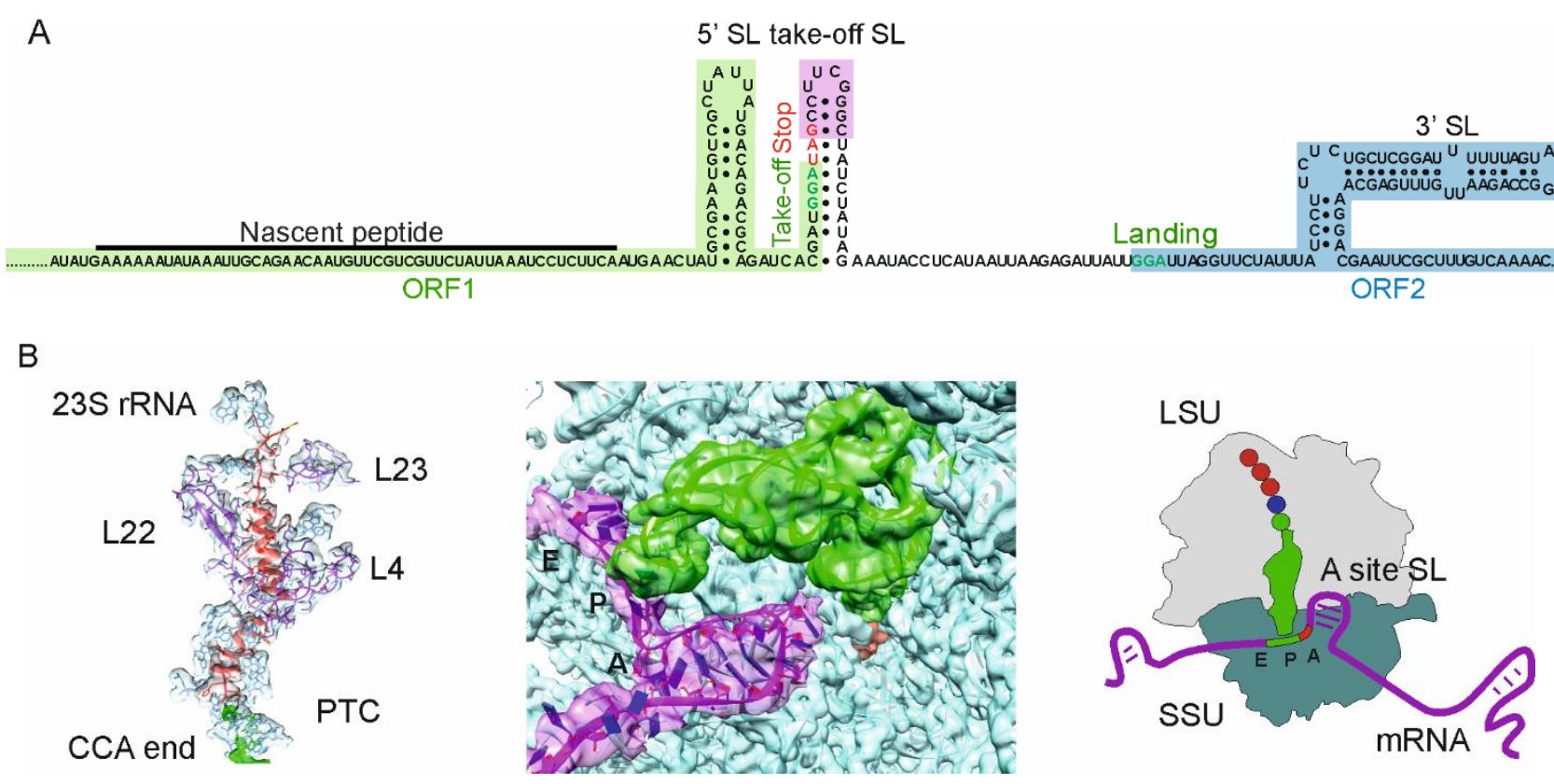

Figure 5. Schematic overview of the bypassing elements in the mRNA of bacteriophage T4 gene 60 (A) The gene 60 mRNA contains all the key signals required for the efficient bypassing: the encoded nascent peptide sequence, the SL elements upstream (5'SL) and downstream (3'SL) of the take-off site, as well as the take-off SL. (B) From left to right: multiple contacts of the nascent peptide with the ribosomal exit tunnel wall and the formation of the short mRNA SL in the A site during ribosomal pausing at the takeoff site shown by cryo-EM structure (Agirrezabala et al., 2017). The right panel shows a schematic representation of the ribosome paused at the take-off site (Rodnina et al., 2019).

Recent biochemical, single-molecule and structural studies provide valuable insights into how translational bypassing works. The initially rapid translation slows down as the ribosome approaches the end of ORF1, which is followed by ribosomal pausing on the take-off site (Chen et al., 2015). The slowdown is presumably caused by the unwinding of the mRNA SL structures by the ribosome and interactions of the nascent peptide chain 
Introduction

with the LSU polypeptide exit tunnel, which was also demonstrated by a cryo-EM structure (Agirrezabala et al., 2017). The nascent peptide forms numerous contacts with the ribosomal polypeptide exit tunnel wall and keeps the peptidyl-tRNA on the ribosome. In addition, the interactions of the nascent peptide residues with the ribosome induce a non-canonical rolled ribosomal state with the PTC in a non-active conformation (Fig.5B). When the ribosome is trapped in this state, it is protected from premature termination and from read-through at the take-off site. Notably, such a non-canonical ribosomal state with inactivated PTC was also detected in a high-resolution structure of SecM-arrested ribosomes (Zhang et al., 2015). Recent smFRET studies reported additional dynamic signatures similar for the SecM and gene 60 translation, namely gradually increased lifetimes of rotated and non-rotated states as the ribosomes approach the SecM stalling sequence or the take-off site (Tsai et al., 2014). Moreover, the long rotated-state pause at the take-off site was suggested to be reminiscent of the hyper-rotated state seen during uncoupled translocation in $d n a X-1$ frameshifting (Chen et al., 2014; Quin et al., 2014; Chen et al., 2015). Together cryo-EM (rolled) and smFRET (hyper-rotated) results may reflect the same non-canonical state of the ribosome obtained upon different experimental conditions, although this assumption would require further corroboration.

Another remarkable feature of the gene 60 take-off complex revealed by the cryo-EM study is a short 12 nt-long SL that occupies the decoding site of the SSU (Fig.5B) and dynamic structures at the 5' and 3' end of the mRNA emerging from the mRNA-binding cleft. The A-site SL hinders the access of the translation termination factor or nearcognate aa-tRNA into the decoding center, which explains why premature termination or read-through does not occur at the take-off site (Agirrezabala et al., 2017). The 5'SL most likely begins to fold when the ribosome moves along the mRNA, thereby forcing the ribosome to slide towards the landing site. The 3'SL downstream of the landing site most probably helps to position the ribosome on the landing site and to resume translation. However, what causes the forward direction of sliding from the take-off site and whether the $12 \mathrm{nt}$ SL has only a protective function remained unclear. 
The landing of the ribosome after successful sliding through the non-coding gap remains the least studied part of bypassing. Previous studies provided a glimpse of the timing of sliding and the adopted rotated conformation of the ribosome before the resumption of translation. Moreover, this study suggests that after sliding the ribosome lands and scans the segment of the non-coding gap to find the best stable landing site (Chen et al., 2015). Despite the 50 nt-long distance between take-off and landing sites, to separate take-off, sliding and landing in time is experimentally challenging due to the high rate of the bypassing process, although the recently reported temperature dependence of bypassing can be used to simplify the experimental system. At low temperatures, the ribosomal pausing on the take-off site is converted to temperatureinduced stalling. This stalling is reversible, which allows the purification of stalled take-off complexes and further activation upon rising the temperature (Agirrezabala et al., 2017). This finding brings new prospectives to bypassing regulation and mechanism studies.

Early in vivo data suggested that r-protein L9 plays a role in translational fidelity and serves to maintain the reading frame during translational pauses. A lack of L9 causes increased frameshifting, miscoding and non-programmed translational bypassing in a number of scenarios (Seidman et al., 2011; Atkins et al., 2016; Smith et al., 2019). Furthermore, mutations in L9 cause the bypassing of the stop codon in gene 60 mRNA when the stop codon is immediately flanked with the GGA codon in the absence of the gap and the main SL structure (Herr et al., 2001). In this case, the gene 60 bypassing is spontaneous and designated as stop-hopping. L9 defects could stimulate ribosome sliding in vivo by increasing the mRNA movement through the ribosome (Seidman et al., 2011; Herr, et al., 2001). L9 is located next to proteins L1, L2, L19, and L28 and may play an architectural role within the ribosome, perhaps as a so-called "molecular strut" serving to stabilize a particular conformation of the 23S RNA and to ensure the normal course of peptide synthesis (Hoffman et al., 1994; Lillemoen et al., 1997).

Because L9 binds at the L1 stalk base near the $E$ site, it was proposed to function during translational pauses as the interface between neighboring ribosomes within a polysome, an assembly of two or more consecutive ribosomes on an mRNA (Smith et al., 
Introduction

2019). Studying ribosomes in the context of polysomes may provide additional insights regarding their spatial orientation in a living cell. Neighboring ribosomes can modulate new ribosome loading on the mRNA, which entails changes in the density of the translating ribosomes and their interaction with each other. This may affect the translational efficiency of an mRNA (Andreeva et al., 2018). Analysis of polysomal arrays revealed several types of ribosomal organization along the mRNA track. In the most prevalent ribosomal assembly, the polysome arrays are pseudo helical and the SSU's heads were shown to be juxtaposed (Brandt et al., 2009). In the arrangement termed "topto-top" (t-t), the SSUs of neighboring ribosomes contact each other in such a way that $r$ protein L9 of a "leading" ribosome projects into the intersubunit space of a "following" ribosome near to its mRNA decoding site (Fig. 6A); this presumably couples the ribosomes within a polysome and might influence the sliding propensity during bypassing (Herr et al., 2001; W. Zhang et al., 2009; Dunkle \& Cate, 2011; Fischer et al., 2015). The second common polysome configuration is named "top to bottom", with the path of mRNA following a more sinusoidal pattern between the ribosomes. On the contrary to $t-t$ arrangement, the position of the neighboring ribosomes within an array seems to be less constrained (Brandt et al., 2009).

A

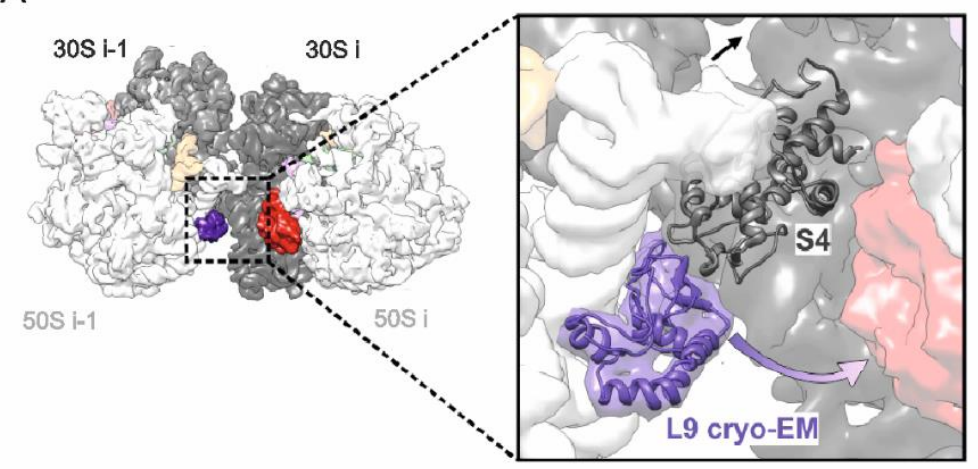

B

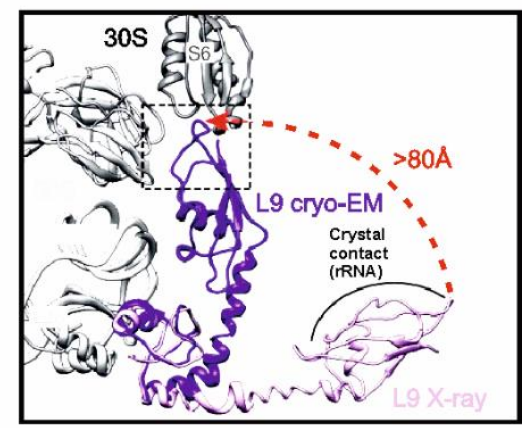

Figure 6. Protein L9 on the ribosome

(A) Model of L9 in the context of major t-t form polysomes. In the arrangement of neighbouring ribosomes the "leading" ribosome is marked as $i-1$, the "following" ribosome marked as $i$ (Fischer et al., 2015; reproduced with permission) (B) L9 in a bent conformation contacting the SSU (L9 cryo-EM, purple) versus the elongated L9 in crystal structures (L9 X-ray, pink). 
Hypothetically, L9 might sense the occupancy of the A site of the neighboring ribosome and in turn influence the L1 stalk/protuberance movement, which indirectly helps to liberate the mRNA for forward mRNA slippage (Wills et al., 2008). Recent studies of -1PRF in the context of polysomes discovered that ribosomes lacking L9 are compacted closer together during collisions and the deletion of L9 caused an increase in -1PRF by individual ribosomes (Smith et al., 2019). Notably, in the cryo-EM structure of a ribosome-EF-Tu complex the L9 is not extended, and its RNA binding domain contacts the SSU within the same ribosome (Fig. 6B) (Fischer et al., 2015). Due to ambiguous Xray and cryo-EM data regarding the location of L9 during translation (Selmer et al., 2012; Noeske et al., 2014; Fischer et al., 2015), most of its rapidly fluctuates between compact and extended conformations and the mechanism of its potential regulator activity remains intriguing. 
Introduction

\subsection{Scope of the thesis}

This work aimed to study the mechanism of programmed bypassing initiation and to identify the key component responsible for the directional sliding of the ribosomal on the gene 60 mRNA of bacteriophage T4. We set out to identify whether bypassing is initiated by the temperature-driven structural rearrangements of the gene 60 mRNA or by the action of a particular translational component using in vitro reconstituted $E$. coli translation system with various reporters. To reveal a connection between ribosomal states adopted during the take-off pausing, we visualized ribosome conformations during bypassing by single-molecule TIRF microscopy. We used stalled take-off complexes formed with fluorescence-labeled ribosomes and monitored ribosomal dynamics upon various experimental conditions. We also investigated potential regulator activity of LSU r-protein L9 during bypassing in vitro upon mono- and polyribosome formation.

Our results show that programmed bypassing in vitro is triggered by the EF-G action and is modulated by temperature-dependent conformational dynamics of the decoding center. Besides, we show that the bypassing efficiency depends on the density of ribosomes on a given mRNA, rather than by the previously suggested action of L9. Our results provide novel insights into the gene 60 mRNA bypassing mechanism and regulation. 
Results

\section{Results}

\subsection{In vitro reconstituted $E$. coli translation system and product analysis}

To study translational bypassing on the gene 60 mRNA of bacteriophage T4, we used a fully reconstituted in vitro translation system from E. coli. It consists of purified $70 \mathrm{~S}$ ribosomes, gene $60 \mathrm{mRNA}$, aminoacylated tRNAs, and translation initiation and elongation factors. The translation was performed upon mixing initiation complexes 70SmRNA-BODIPY-Met-tRNA ${ }^{\text {fMet }}$ (IC) with the ternary EF-Tu-GTP-aa-tRNA complex in the presence of EF-G-GTP. The IC was formed using fluorescence-labeled initiator tRNA to visualize the translation products carrying a fluorescent reporter on the $\mathrm{N}$-terminus which can be visualized on SDS-PAGE gels (Fig. 7). There are two main products of gene 60 translation. The first product (stop) corresponds to ORF1 and appears when the ribosome stops at the take-off GGA codon. The second product is the bypassing (byp) product, which is synthesized when the ribosome successfully lands at the landing GGA codon and translates ORF2 to the end. Using previously developed and optimized translation conditions (Samatova et al., 2014), quantification of gel band intensities gave the efficiency of bypassing at about $50-60 \%$, which is in the range of reported in vivo values (Maldonado \& Herr, 1998). To obtain ribosome complexes stalled at the take-off site with bound peptidyl-tRNA ${ }^{\text {Gly }}$ GGA and nascent peptide, we used a previously reported temperature dependence feature of gene 60 bypassing (Agirrezabala et al., 2017). At $10^{\circ} \mathrm{C}$ gene $60 \mathrm{mRNA}$ is translated up to the take-off codon, but the full-length byp product is not synthesized; in contrast, at $37^{\circ} \mathrm{C}$ the efficiency of bypassing is high and saturated (Fig. 7). When a take-off complex stalled at $10^{\circ} \mathrm{C}$ is incubated with the rest of the translation mixture at $37^{\circ} \mathrm{C}$, the ribosomes resume bypassing, showing that ribosomal complexes initially stalled at low temperature retain their activity. This feature allowed us to purify take-off complexes stalled at $10^{\circ} \mathrm{C}$ using size-exclusion chromatography and use them in further studies on the initiation of bypassing as well as ribosome dynamics. 
Results

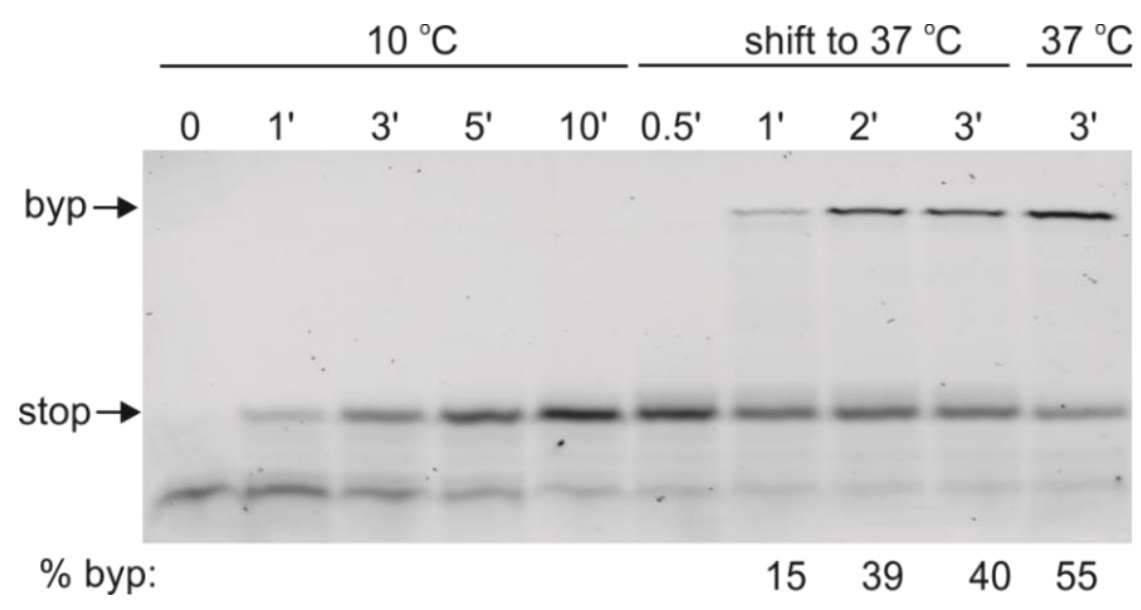

Figure 7. Formation of peptide products upon translation of gene 60 mRNA at the different temperatures The translation products of gene 60 were separated by SDS-PAGE and visualized using a fluorescent reporter, BODIPY FL, incorporated co-translationally at the $\mathrm{N}$ terminus of the nascent peptide. When in vitro translation is carried out at $10^{\circ} \mathrm{C}$, only the ORF1 product (stop) is synthesized. Shifting the temperature to $37^{\circ} \mathrm{C}$ activates bypassing and leads to the ORF1+ORF2 product (byp) synthesis. The byp ( 15 kDa) and stop ( 5 kDa).products are indicated with arrows (Agirrezabala et al., 2017).

To study the conformational dynamics of the ribosome during bypassing, we used a single-molecule Förster resonance energy transfer (smFRET) approach and utilized a FRET assay previously developed by $\mathrm{H}$. Noller and validated by several groups (Ermolenko et al., 2007; Cornish at al., 2008; Sharma et al., 2016; Adio et al., 2018). We introduced fluorescent cyanine reporters Cy5 and Сy3 at unique cysteine residues in the r-proteins S6 and L9, respectively. Cys residues were introduced by site-directed mutagenesis at position 41 in S6 replacing aspartic acid (D41C) and at position 11 in L9 replacing asparagine (N11C). SSU and LSU carrying labeled Cy5-S6 and Cy3-L9 were prepared by in vitro reconstitution by mixing subunits prepared from strains lacking S6 $(\Delta \mathrm{S} 6)$ or $\mathrm{L9}(\Delta \mathrm{L} 9)$ with an excess of fluorescence-labeled protein $\mathrm{S} 6$ or $\mathrm{L9}$, respectively. The labeled ribosomes were used to form take-off complexes stalled at $10^{\circ} \mathrm{C}$.

\subsection{Studying the effect of elongation factors on bypassing initiation}

Our first goal was to answer the question of whether initiation of bypassing is facilitated predominantly by the structural dynamics of gene $60 \mathrm{mRNA}$, or by a component of the in vitro translation system serving as a trigger for the ribosome take-off and 
subsequent sliding towards the landing site. The minimal translation system consists of the ribosome, the mRNA, the pool of aa-tRNA and protein factors, including IF1-3, EF-Tu and EF-G. Termination factors are not included to simplify the system and avoid possible competition between bypassing and termination on the UAG stop codon adjacent to the take-off site. This allowed us to achieve the highest possible bypassing efficiency in vitro; however, as had been previously shown, the addition of RF1 does not result in a dramatic in bypassing efficiency (Agirrezabala et al., 2017). Initiation factors do not influence the bypassing efficiency, which was tested by in vitro translation assays using IC purified from initiation factors (E. Samatova, personal communication). Because both elongation factors are essential for the translation process, it was not feasible to exclude one or the other from the translation mixture. Therefore, we first compared the dependence of translation and bypassing efficiency on the EF-G concentration by performing an EF-G titration (Fig. 8 ) in a wide range of concentrations $(0.004-0.8 \mu \mathrm{M})$. 
Results

A

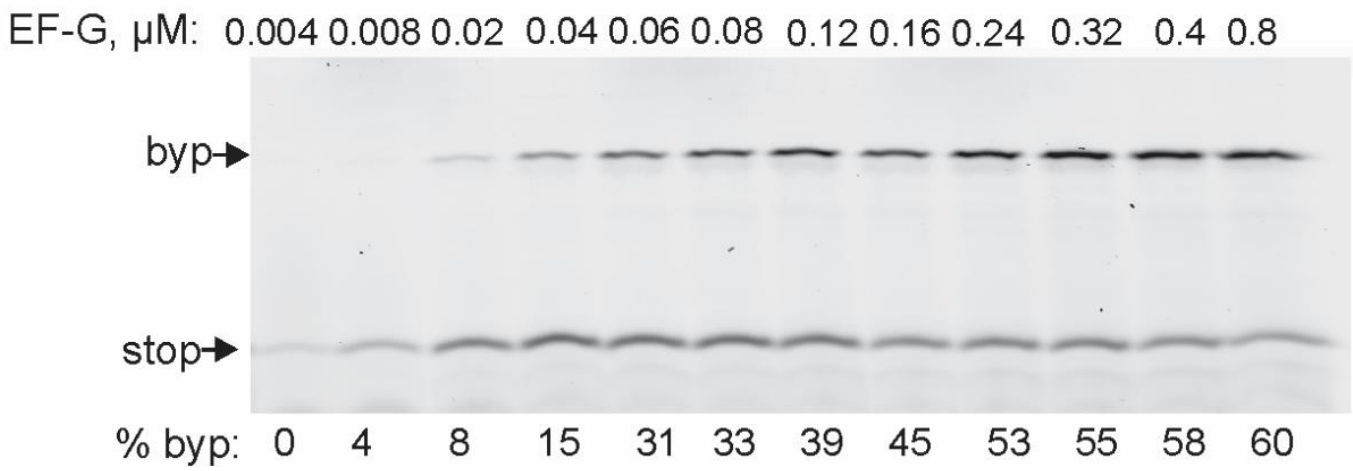

B

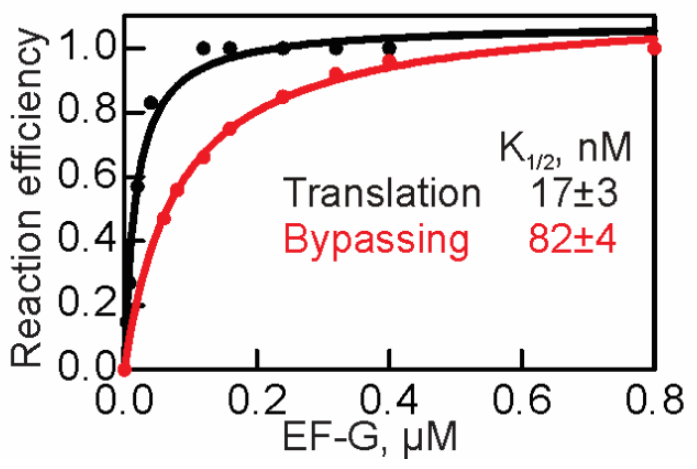

Figure 8. Translation and bypassing at different EF-G concentrations

(A) Formation of peptide products upon translation of gene $60 \mathrm{mRNA}$ at different concentrations of EF-G. The byp and stop products are indicated with arrows. (B) Quantification of the EF-G dependence of bypassing. Bypassing efficiencies were calculated from the band intensities of the byp and stop products as a ratio between the byp band and the sum of the translation products. Titration curves fit according to the hyperbolic model are shown as continuous lines.

The overall translation and bypassing efficiencies increase with the EF-G concentration (Fig. 8A). To estimate the impact of EF-G on bypassing vs. overall translation efficiencies, we determined the concentrations of EF-G at the half-maximum of translation and of bypassing (Fig. 8B). The EF-G concentration required for efficient bypassing was almost 5 times higher than that needed for regular translation. This observation reflects the higher affinity of EF-G for translating ribosomes vs. bypassing ribosomes. To test whether also at higher EF-Tu concentration the bypassing efficiency increases, we performed a titration of EF-G $(0.008-0.4 \mu \mathrm{M})$ with standard $(50 \mu \mathrm{M})$ and 
Results

twice as high EF-Tu concentration (Fig. 9A). In contrast to EF-G, the increase of the EFTu concentration reduced the bypassing efficiency, while the translation efficiency as such, i.e. the translation of ORF1, was not affected (Fig. 9B-C). This might be explained by the fact, that both EF-Tu and EF-G bind to the ribosomal A site, a higher EF-Tu concentration causes a lower probability for EF-G binding. Bypassing appears to be more sensitive to the ratio of EF-T and EF-G than regular translation. Thus, EF-G appeared to affect bypassing, but in order to deduce its exact role, we needed to separate the takeoff and landing events from the regular translation process of the ORF2.

A

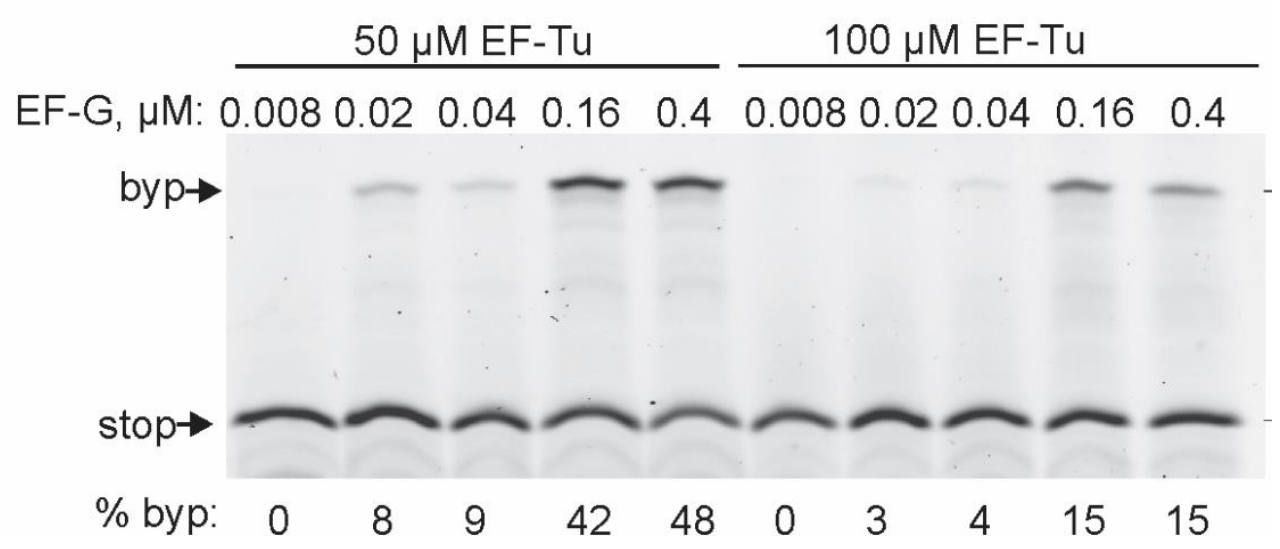

B

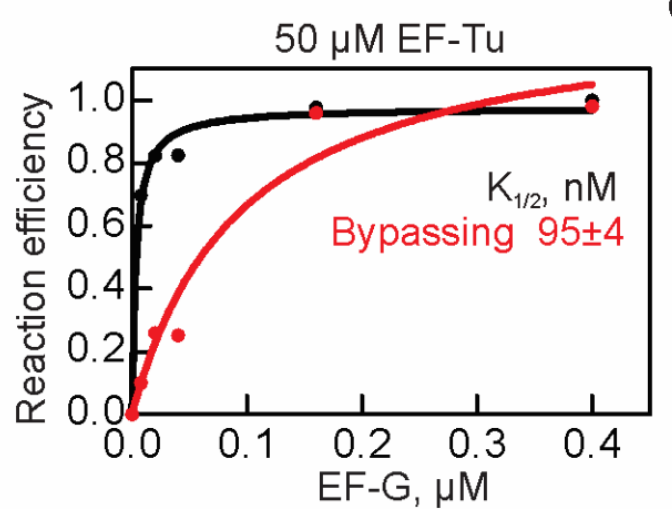

C

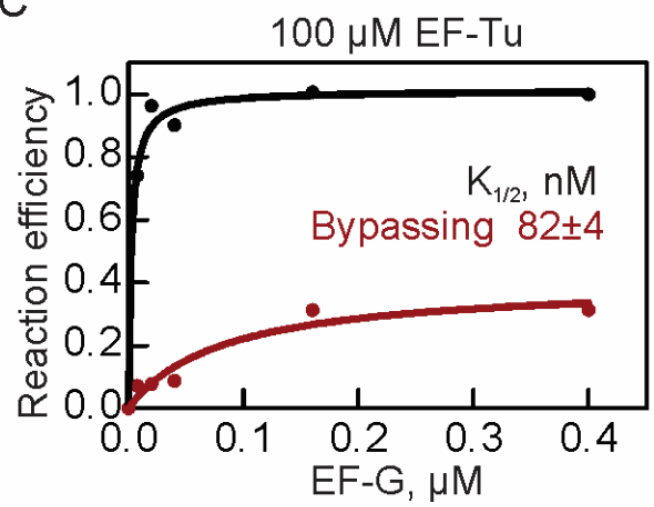

Figure 9. Translation and bypassing at different EF-G and EF-Tu concentrations

(A) Formation of peptide products upon translation of gene $60 \mathrm{mRNA}$ at different concentrations of EF-G with standard and elevated EF-Tu concentrations. The byp and stop products are indicated with arrows.

(B) Quantification of the EF-G concentration dependence with standard (B) and elevated (C) EF-Tu concentrations. EF-Tu concentrations. Translation efficiencies were normalized to the maximum translation efficiencies at $50 \mu \mathrm{M}$ and $100 \mu \mathrm{M}$ EF-Tu concentrations respectively. Bypassing efficiencies were 
Results

normalized to the maximum bypassing efficiency at $0.4 \mu \mathrm{M} \mathrm{EF-G}$ and $50 \mu \mathrm{M}$ EF-Tu concentrations. Titration curves fit according to the hyperbolic model are shown as continuous lines.

\subsection{Monitoring bypassing initiation using a $\left[{ }^{14} \mathrm{C}\right]$ Leu incorporation assay}

During bypassing on the gene $60 \mathrm{mRNA}$ the ribosome takes off and lands on identical glycine GGA codons. Interactions of the nascent peptide anchor the peptidyl-tRNA ${ }^{\text {Gly }}{ }_{\text {GGA }}$ on the ribosome during sliding (Agirrezabala et al., 2017), this tRNA binds to the GGA landing codon and translation resumes by reading the Leu codon UUA adjacent to the landing GGA codon. Therefore, bypassing is considered to be successful if the Leu is incorporated into the growing nascent peptide chain. To determine the exact role of EF$G$ in bypassing, we developed an assay to monitor the reading of the UUA codon and $\left[{ }^{14} \mathrm{C}\right]$ Leu incorporation into the nascent peptide as a readout for ribosome landing. First, we prepared stalled take-off complex using $f\left[{ }^{3} \mathrm{H}\right]$ Met and purified the complex from the translational components. This complex did not contain $\left[{ }^{14} \mathrm{C}\right] \mathrm{Leu}$ (Fig. 10A, 10F). In the next step we mixed the stalled take-off complex with EF-Tu-GTP- $\left[{ }^{14} \mathrm{C}\right]$ Leu-tRNA ${ }^{\text {Leu }_{U} \text { UA }}$ in the presence or absence of EF-G and monitored the $\left[{ }^{14} \mathrm{C}\right]$ Leu signal in the polypeptide product (Fig. 10A-F). Because radioactive Leu can be incorporated into the nascent peptide chain only if the ribosome took off and bypassed the non-coding gap, ${ }^{14} \mathrm{C}$ counts provide a quantitative readout for bypassing. Of note, we only detected the extent, rather than the kinetics of $\left[{ }^{14} \mathrm{C}\right]$ Leu insertion into the polypeptide due to a long incubation time (2 min) and saturating concentration of EF-G $(2 \mu \mathrm{M})$. We did not observe $\left[{ }^{14} \mathrm{C}\right] \mathrm{Leu}$ incorporation after mixing the take-off complex with ternary complex in the absence of EF-G (Fig. 10B, 10F), whereas upon adding EF-G the incorporation of Leu reached $\sim 30 \%$ (Fig. 10C, 10F), thus suggesting that bypassing was indeed triggered solely by the action of EF-G. Next, we tested the ability of used EF-G mutants with replacements of key amino acids at the tip of domain IV (H583K and Q507D) to trigger bypassing. These mutations in EF-G were shown to slow down translocation, whereas the binding of the factor to the ribosome or its ability to hydrolyze GTP were not affected (Savelsbergh et al., 2000; Peng et al., 2019). Also with those mutants $\left[{ }^{14} \mathrm{C}\right]$ Leu was efficiently incorporated. (Fig. 10D-F). The results of these experiments suggest that the act of EF-G binding to the ribosomal A site is essential for bypassing. 
A

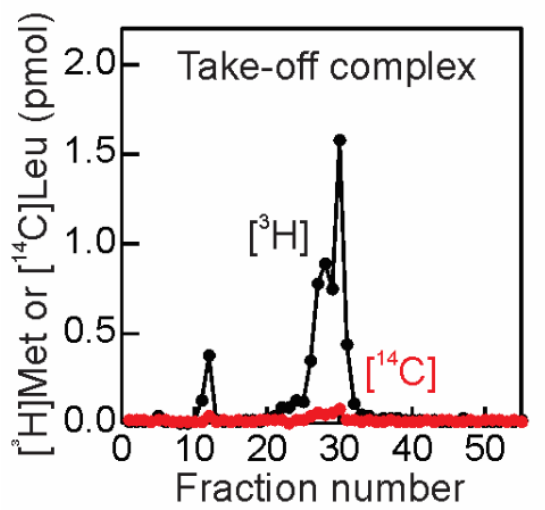

$\mathrm{D}$

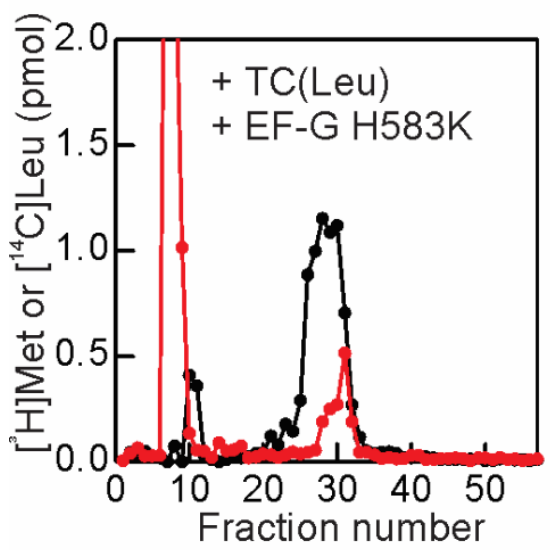

B

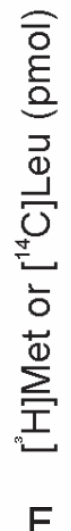

$\mathrm{F}$

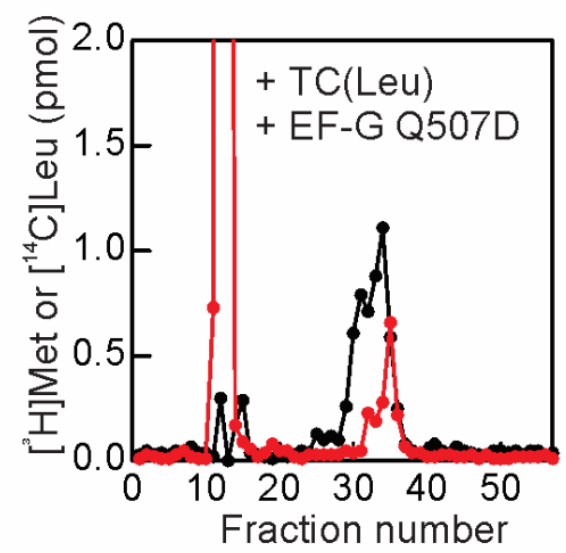

C

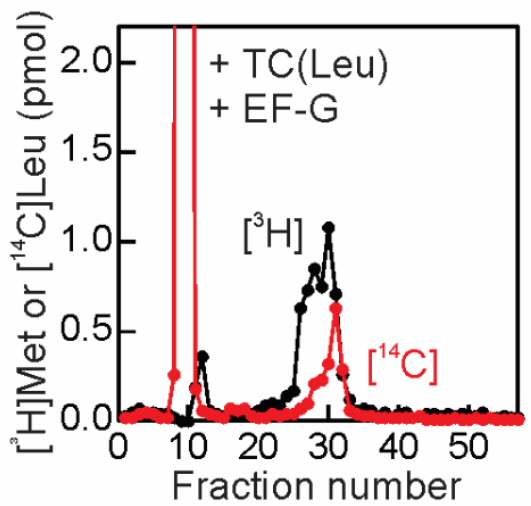

E

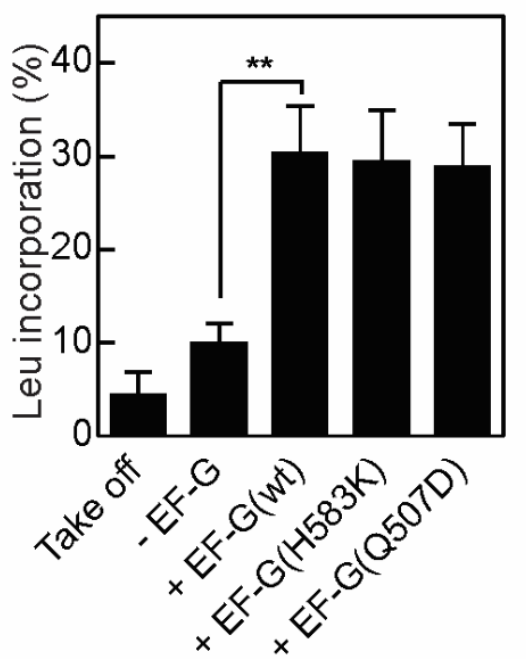

Figure 10. Identification of the factor essential for bypassing

(A) High-Performance Liquid Chromatography (HPLC) profile of the take-off and landing products separation. Translation products bound to the ribosome in the purified take-off complex visualized by $\left[{ }^{3} \mathrm{H}\right] \mathrm{Met}$ (black) and $\left[{ }^{14} \mathrm{C}\right] \mathrm{Leu}$ (red) counting. The first peaks correspond to the free $\left[{ }^{3} \mathrm{H}\right] \mathrm{Met}$ $/\left[{ }^{14} \mathrm{C}\right]$ Leu. Take-off complexes were prepared and purified from translational components at $10^{\circ} \mathrm{C}$ and transferred to $37^{\circ} \mathrm{C}$;

(B) As in (A), but with the addition of EF-Tu-GTP- $\left[{ }^{14} \mathrm{C}\right]$ Leu-tRNA complementary to the UUA codon in the absence of EF-G;

(C) As in (A), but with the addition of EF-Tu-GTP- $\left[{ }^{14} \mathrm{C}\right]$ Leu-tRNA and EF-G;

(D) As in (A), but with the addition of EF-Tu-GTP- $\left[{ }^{14} \mathrm{C}\right]$ Leu-tRNA and EF-G (H583K);

(E) As in (A), but with the addition of EF-Tu-GTP-[14 C]Leu-tRNA EF-G (Q507D);

(F) Efficiency of $\left[{ }^{14} \mathrm{C}\right]$ Leu incorporation measured under various conditions. Error bars show standart deviation (SD) obtained from three replicates. ${ }^{* *} \mathrm{P}<0.005$ by Student's two-tailed unpaired t-test. 
Results

\subsection{GTP expenditure during ribosomal sliding on the non-coding gap}

Our finding that EF-G plays a key role during bypassing prompted us to test whether the role of EF-G is limited to a single action in triggering bypassing or it also has additional functions in maintaining the directional sliding. Notably, rapid GTP hydrolysis by EF-G is activated by the ribosome, EF-G alone lacks GTPase activity. It can yet interact with the vacant or stalled ribosomes and hydrolyze GTP uncoupled from movement (Cunha et al., 2013; Maracci \& Rodnina, 2016). We estimated the amount of GTP hydrolyzed by EF-G during bypassing by mixing the stalled take-off complex with $\left[\gamma^{32} \mathrm{P}\right] \mathrm{GTP}$ and using thinlayer chromatography (TLC) to distinguish between unhydrolyzed GTP and free Pi and to quantify the extent of GTP hydrolysis. To distinguish the vacant/stalled ribosome fraction from the ribosomes active in bypassing, we compared GTP hydrolysis on the standard take-off complexes with complexes in which bypassing was not possible, because they were formed on an mRNA truncated after the first stop codon of ORF1 (Fig. $11 \mathrm{~A}-\mathrm{B})$. We observed a higher amount of hydrolyzed GTP in the bypassing complexes than in the case of the vacant/ stalled ribosomes. The extent of GTP hydrolysis during bypassing was calculated by subtracting the amount of GTP hydrolyzed on vacant/stalled ribosomes from the amount of GTP hydrolyzed on the wild type (WT) gene 60 mRNA take-off complexes (Fig. 11C). We then compared it to the bypassing kinetics performed by the $\left[{ }^{14} \mathrm{C}\right]$ Leu incorporation assay carried out at the same concentrations and time points as the $\left[\gamma^{32}\right.$ P]GTP assay. In these experiments, the bypassing efficiency ( $\left.20 \%\right)$ was somewhat lower than in all other experiments because of the limiting concentrations of EF-G $(0.1 \mu \mathrm{M}$ instead of $2 \mu \mathrm{M})$ and GTP $(10 \mu \mathrm{M}$ instead of $1 \mathrm{mM})$ chosen to maximize the sensitivity of the assay to GTP hydrolysis. The rate of $\left[{ }^{14} \mathrm{C}\right]$ Leu incorporation and of GTP hydrolysis during bypassing were almost identical and both could be described by a 
Results

single-exponential curve, suggesting a single rate-limiting step for GTP hydrolysis and bypassing (Fig. 11D).

A

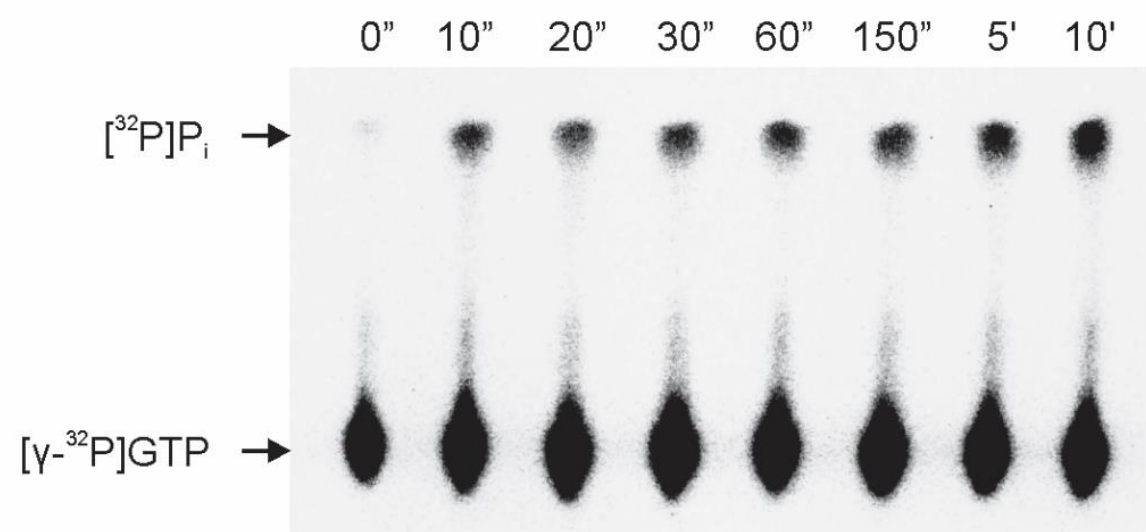

B

0" $10 " 20 " 30 " 60 " 150 " 0^{\prime \prime} \quad 5^{\prime} \quad 10^{\prime}$

$\left.{ }^{32} \mathrm{P}\right] \mathrm{P}_{\mathrm{i}} \rightarrow$

$\left[\mathrm{Y}^{32} \mathrm{P}\right] \mathrm{GTP} \rightarrow \mathrm{O}$
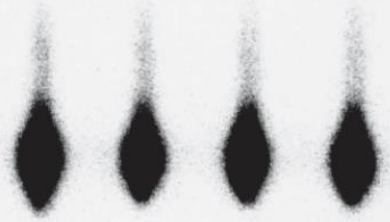

C
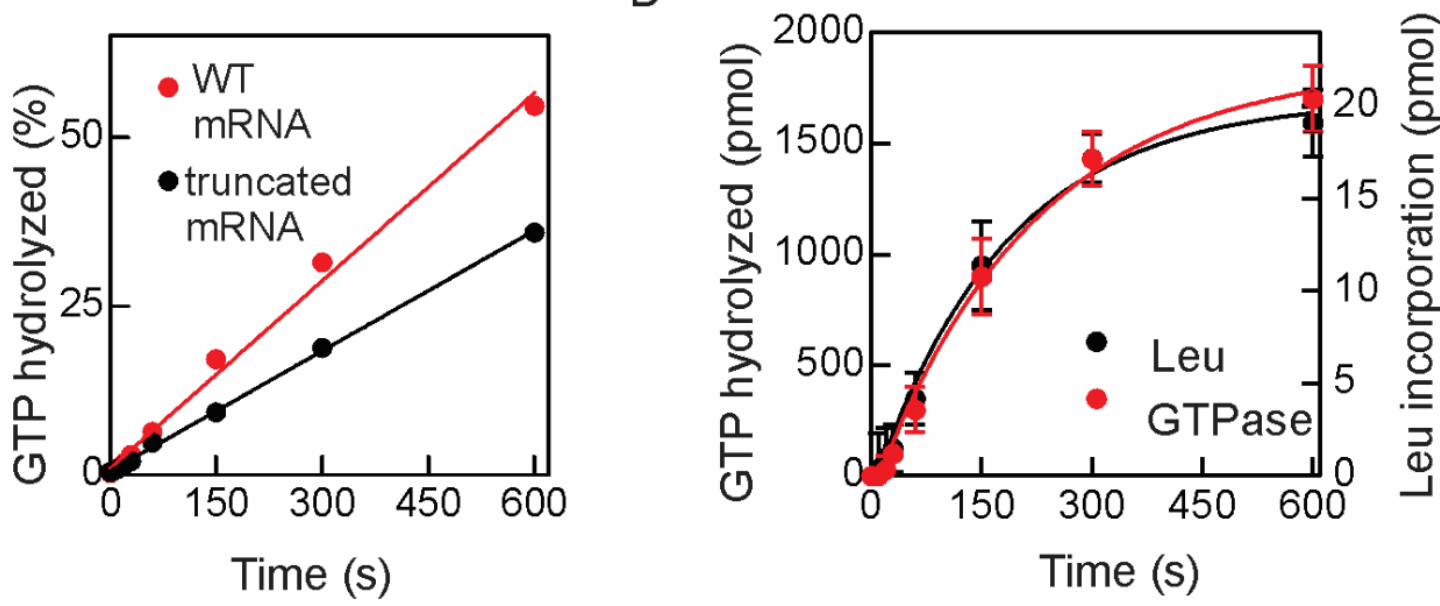

Figure 11. Time course of GTP hydrolysis during bypassing

GTP hydrolyzed on complexes formed on WT gene 60 (A) and truncated mRNA (B). Pi was separated from GTP by TLC, the products are indicated with arrows. Incubation times ( 0 seconds -10 minutes) are shown above. (C) Example of GTP hydrolysis kinetics quantification. The extent of hydrolysed GTP was calculated 
Results

from the intensities of the spots as the ratio of the $P_{i}$ divided by the sum of the $P_{i}$ and GTPband intensities multiplied by $100 \%$. (D) GTPase activity of EF-G during bypassing and the kinetics of bypassing measured by $\left[{ }^{14} \mathrm{C}\right]$ Leu incorporation. Error bars show standard deviation (SD) obtained from three replicates. Curves are fit according to the single-exponential model.

When normalized to the number of ribosomes that successfully reached the landing site and assuming that GTP is hydrolyzed the whole time the ribosome slides along the mRNA, EF-G appeared to hydrolyze on average 90 molecules of GTP for each ribosome that completed bypassing.

During regular translation about one GTP molecule is hydrolyzed to move the ribosome by three nucleotides (one codon), but on the bypassing ribosomes EF-G hydrolyzes substantially more GTP than expected for a single take-off event. This indicates multiple EF-G binding and GTP hydrolysis events during sliding. Taking into account that the non-coding gap is 50 nucleotides long, EF-G hydrolyzed on average 1.8 molecules of GTP per nucleotide of the sliding sequence. Such a GTP hydrolysis activity may be needed to maintain a ribosome conformation required for sliding or to facilitate the directional forward movement of the ribosome in a manner similar to the power-stroke action of EF-G during translocation (Chen et al., 2016; Salsi et al., 2015).

\subsection{The role of the A-site mRNA stem-loop}

Next, we asked whether the A-site SL has a role in the initiation of bypassing. If the stability of the A site hairpin during take-off pausing is the limiting factor for bypassing, we presumed that the bypassing efficiency depends on the SL folding energy. A-site SL should be disrupted to alleviate the pausing on the take-off site and to allow for ribosome movement towards the landing site. Hypothetically, a weaker SL should be easier to unfold. The formation of an SL in the A site with lower folding energy should facilitate the forward movement of the ribosome from the take-off site and hence result in elevated bypassing efficiency. 
We introduced a set of mutations into the gene 60 mRNA (Fig. 12A) that either disrupt or stabilize base pairing in the hairpin and were shown to change the bypassing efficiency in vivo (Herbst et al., 1994) and measured how these mutations affect in vitro bypassing efficiency. We observed, that the bypassing efficiency indeed depends on the hairpin stability, but not in the way we initially expected (Fig. 12B). The bypassing efficiency increased with the SL stability (calculated by mfold software) and was saturated at folding energies below $-17 \mathrm{kcal} / \mathrm{mol}$; further helix stabilization did not elevate the bypassing efficiency further (Fig. 12C). Therefore, a stable SL formed in the A site of the ribosome is required for bypassing.

A

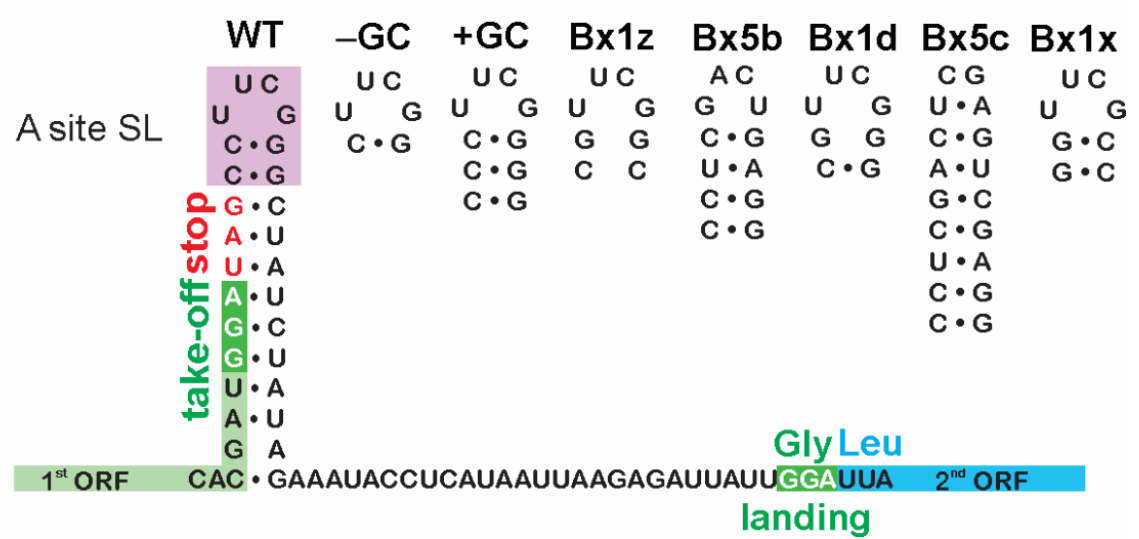

B

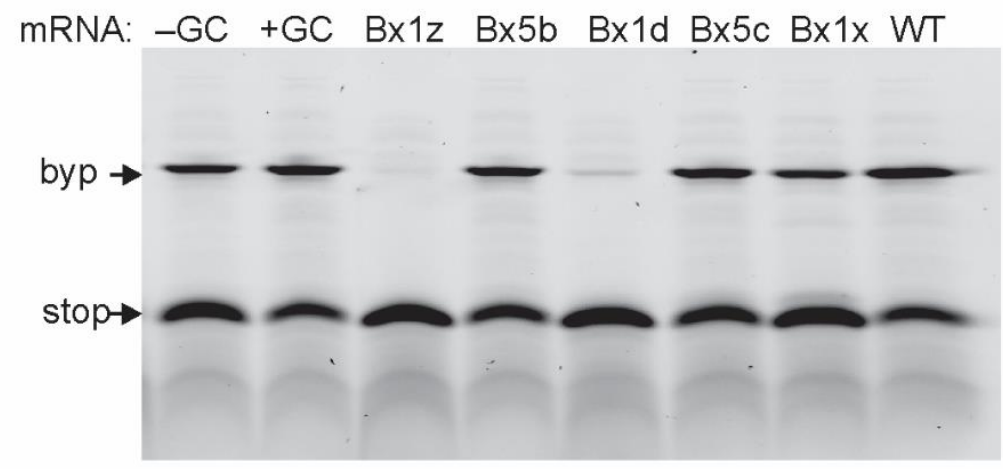

C

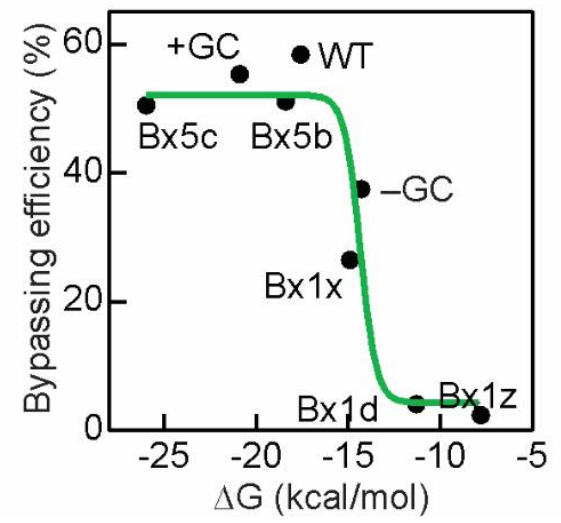

Figure 12. Effect of mutations in the A-site SL on bypassing

(A) The gene $60 \mathrm{mRNA}$ structure. The mutated part of the A-site SL is highlighted in pink; the mutations in the loop are shown above the mRNA sequence. (B) Effect of mutations in A-site SL on bypassing. The byp and stop products are indicated with arrows. The A-site SL mutations are indicated above the gels. (C) Bypassing efficiency at predicted folding energies of the A-site SL mutants. The curve is fit according to the sigmoid model. 
Results

\subsection{Conformational dynamics of the decoding center as a bypassing modulator}

As was described before, bypassing efficiency is temperature-dependent. At low temperatures, the ribosome translates to the take-off site, where it resides in a stalled state until the temperature is increased and further bypassing and ORF2 translation are allowed (Agirrezabala et al., 2017). Such an effect suggests certain temperature-driven transitions within the translation system, which are crucial for bypassing efficiency. The temperature dependence of bypassing has a sigmoidal shape and its inflection point corresponds to the temperature at which the bypassing efficiency reaches half of its maximum value - $\mathrm{T}_{\mathrm{b}}$. For the non-mutated WT gene 60 , we obtained a $\mathrm{T}_{\mathrm{b}}$ of $24.1 \pm 0.2^{\circ} \mathrm{C}$ (Fig. 13, WT).

We sought to understand which component of the translation mixture determines the temperature dependence. Our first assumption was that this effect is related to the temperature-driven folding-unfolding of mRNA structural elements, such as A-site SL and 5'SL. To start with, we compared the bypassing efficiencies for the A-site SL mRNA mutants allowing for bypassing (i.e. with sufficient A-site SL energies) over a temperature range from $10^{\circ} \mathrm{C}$ to $37^{\circ} \mathrm{C}$. Surprisingly, we observed a decrease in bypassing efficiencies (Fig 13A), but no significant change of the $T_{b}$ value in comparison to the WT mRNA (Fig. 2.7B). 
A

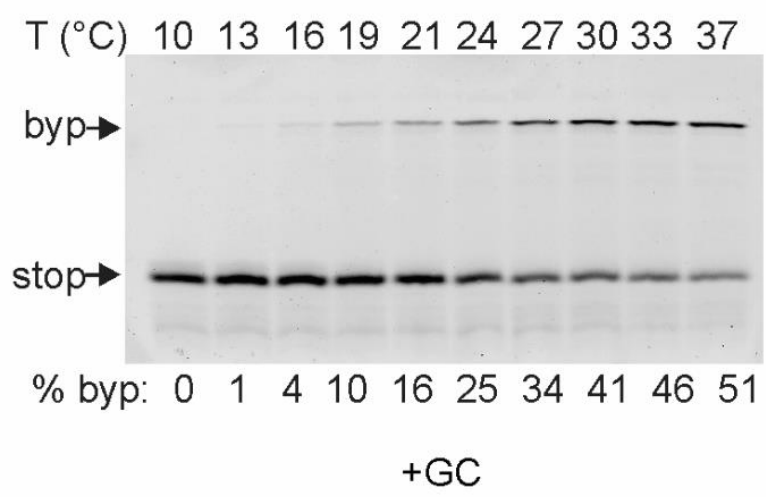

$\mathrm{T}\left({ }^{\circ} \mathrm{C}\right) 10 \quad 13 \quad 16192124 \quad 273033 \quad 37$

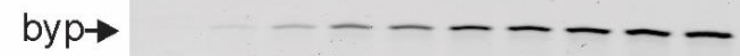

stop $\rightarrow$

\% byp: $0 \quad 2 \quad 5 \quad 11 \quad 17 \quad 2736 \quad 424750$

$\mathrm{B} \times 5 \mathrm{c}$

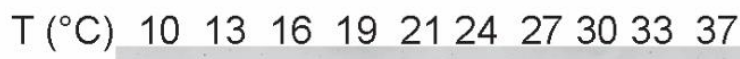

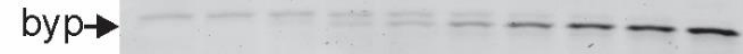

stop $\rightarrow$

$\begin{array}{llllllllll}\text { \% byp: } 0 & 0 & 1 & 6 & 12 & 21 & 31 & 39 & 44 & 49\end{array}$

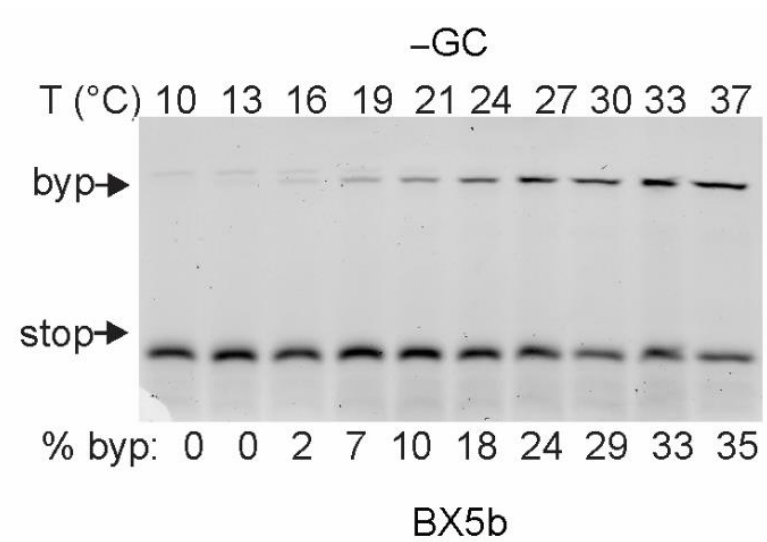

$\mathrm{T}\left({ }^{\circ} \mathrm{C}\right) 10 \quad 13 \quad 16192124 \quad 2730 \quad 33 \quad 37$

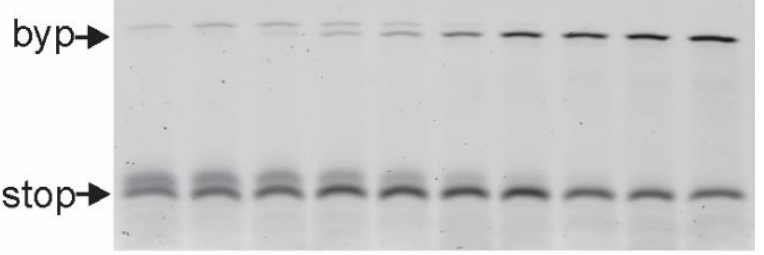

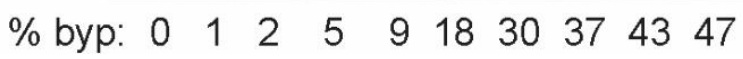

B

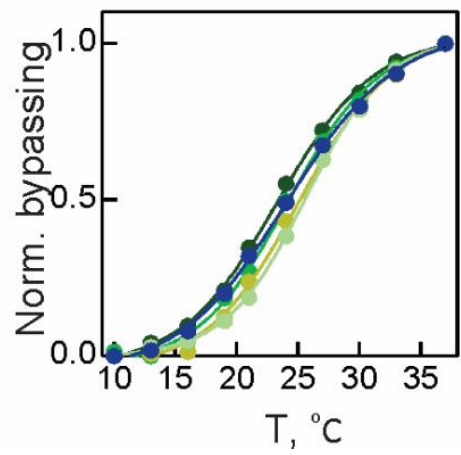

$\mathrm{T}_{\mathrm{b}}\left({ }^{\circ} \mathrm{C}\right)$

-WT $24.1 \pm 0.2$

- $-G C 24.2 \pm 0.3$

- +GC $23.4 \pm 0.2$

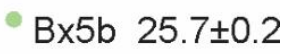

Bx5c 25.0 0.3

Figure 13. The effect of mutations in the A-site SL on the temperature of bypassing

(A) Bypassing at different temperatures for the A-site SL mutants. The byp and stop products are indicated with arrows, the incubation temperatures are indicated above the gels. (B) Example of the temperature dependence of bypassing for the A-site SL mutants quantification; the bypassing efficiency was normalized for the maximum efficiency at $37^{\circ} \mathrm{C}$. $\mathrm{T}_{\mathrm{b}}$ represented by an average value and a standard deviation (SD) obtained from two replicates. Curves are fit according to the sigmoid model.

As a second step, we introduced four mutations that disrupt the mRNA 5'SL structure (4M) and were shown to decrease bypassing efficiency. We also placed compensatory mutations (4MC) that partially rescue bypassing efficiency (Samatova et al., 2014) (Fig. $14 \mathrm{~A})$. Both of the mutations aimed to alter the mRNA structure but did not change the 
Results

encoded amino acid sequence. Despite the 4- and 2-fold decreases in bypassing efficiency for $4 \mathrm{M}$ and $4 \mathrm{MC}$ mutants respectively (Fig. 14B), the $T_{b}$ values were hardly changed for both 5'SL mutant mRNAs, presuming that 5'SL does not contribute to the temperature dependence of bypassing (Fig 14C).

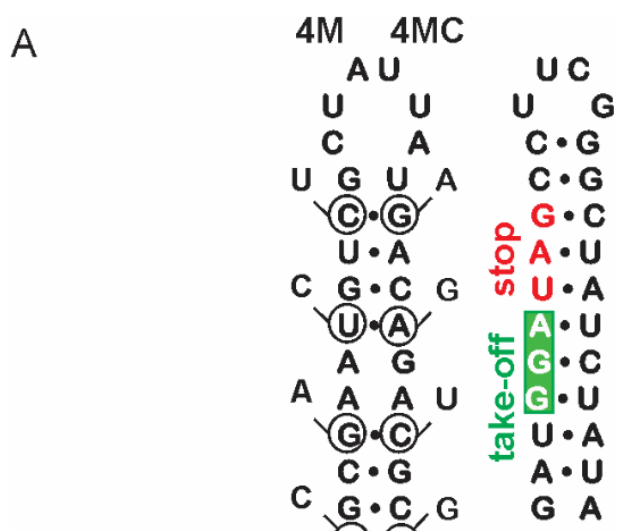

B

-....AACUAU(i) (AGAUCAC • GAAAUACCUCAUAAUUAAGAGAUUAUUGGA $2^{\text {nd }}$ ORF

landing

$4 \mathrm{M}$

$4 \mathrm{MC}$

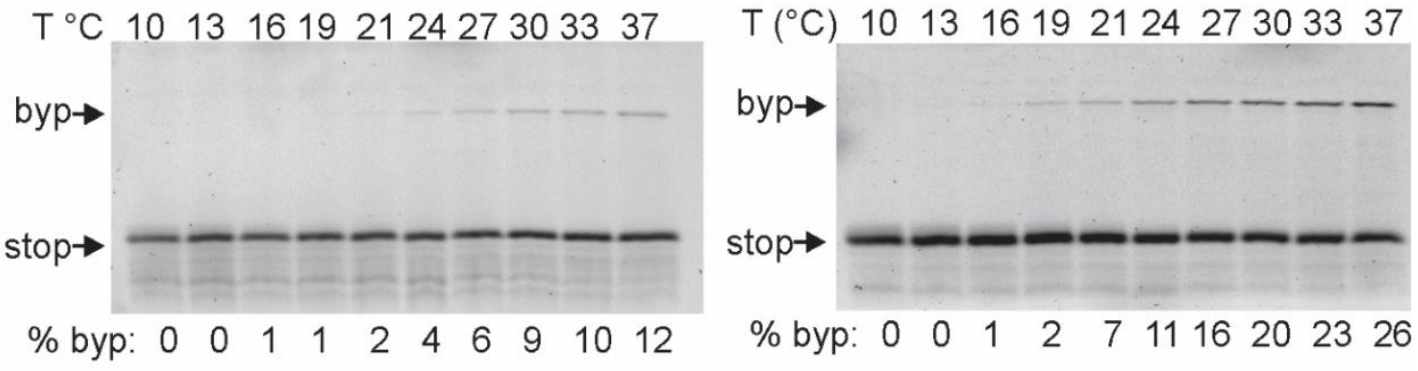

C

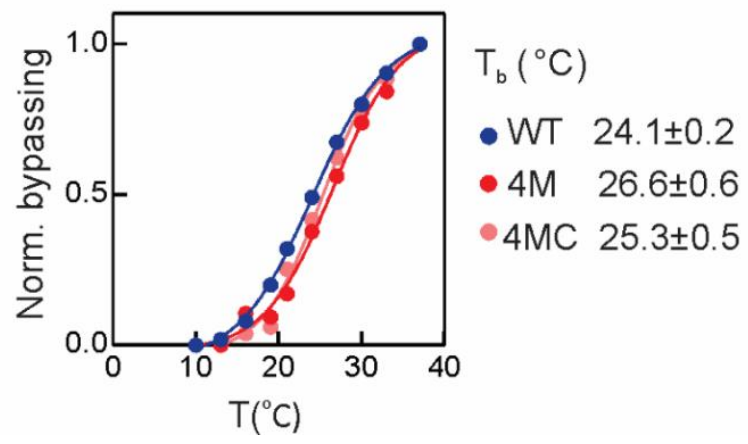

Figure 14. The effect of mutations in the 5 'SL on the temperature dependence of bypassing (A) Mutations introduced to disrupt $(4 \mathrm{M})$ or to restore $(4 \mathrm{MC})$ the putative secondary structure of the 5 'SL; the substitutions are indicated with circles (Samatova et al., 2014). (B) Bypassing at different temperatures for the 5'SL mutants. The byp and stop products are indicated with arrows, the incubation temperatures are 
indicated above the gels. (C) Example of the temperature dependence of bypassing for the 5'SL mutants; the bypassing efficiency was normalized for the maximum efficiency at $37^{\circ} \mathrm{C}$. $T_{b}$ is the temperature at which bypassing is half-maximal. Tb represented by an average value and a standard deviation (SD) obtained from two replicates. Curves are fit according to the sigmoid model.

Another bypassing signal which might be responsible for the temperature dependence of bypassing is provided by the interactions of the nascent peptide with the polypeptide exit tunnel of the ribosome (Weiss et al., 1990; Samatova et al., 2014; Agirrezabala et al., 2017). 
Results

A

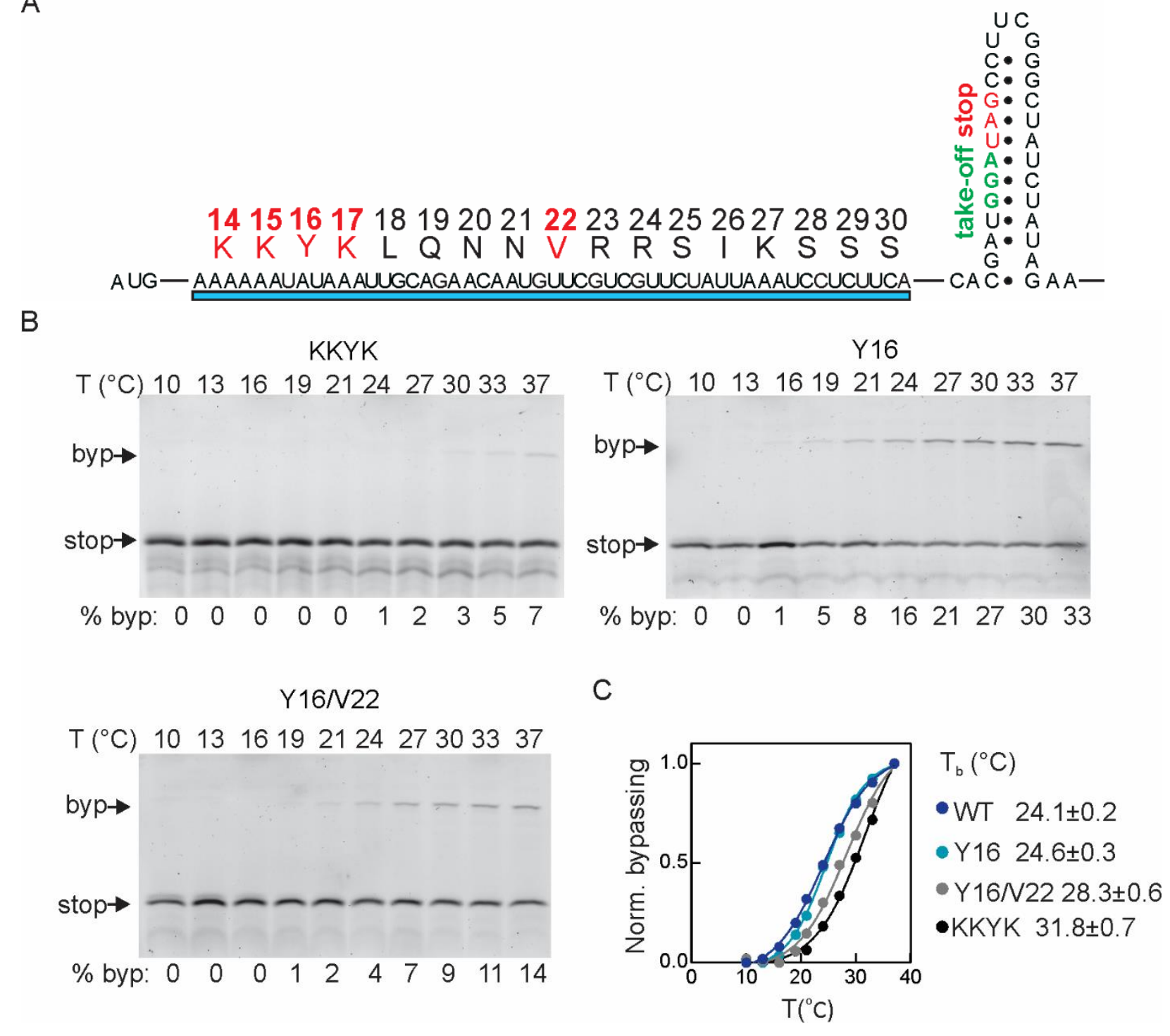

Figure 15. The effect of mutations in the nascent peptide on the temperature dependence of bypassing (A) Schematic of the nascent peptide sequence encoded upstream the take-off site; the amino acid sequence indicated above shows the mutated aminoacids (red) that are known to affect bypassing. (B) Bypassing at different temperatures for the nascent peptide mutants. The byp and stop products are indicated with arrows the incubation temperatures are indicated above the gels. (C) Example of temperature dependence of bypassing for the nascent peptide mutants; the bypassing efficiency was normalized for the maximum efficiency at $37^{\circ} \mathrm{C}$. $T_{b}$ is the temperature at which bypassing is half-maximal. $T_{b}$ represented by an average value and a standard deviation (SD) obtained from two replicates. Curves are fit according to the sigmoid model. 
Replacement of residue Y16 to D (Y16D) or of the essential KKYK motif (amino acids 14 to 17) (Fig. 15A) caused a 1.5 fold reduction of the bypassing efficiency (Fig 15B) but did not alter the temperature dependence curve. A double mutation Y16H/V22D led to a 4-fold decrease of the bypassing efficiency, but at the same time, it elevated the $T_{b}$ value from $24.1^{\circ}$ to $28.3^{\circ} \mathrm{C}$. Replacement of the entire KKYK motif with EEHE aimed to abolish the nascent chain interactions in the ribosomal exit tunnel shown to be crucial for bypassing (Weiss et al., 1990; Chen et al., 2015). It resulted in dramatically decreased bypassing efficiency (7-fold) and even further increase of the $\mathrm{T}_{\mathrm{b}}$ value to $31.8^{\circ} \mathrm{C}$ (Fig. $15 \mathrm{C})$.

\subsection{Ribosome dynamics as a modulator of bypassing efficiency}

Nascent peptide residues are known to affect bypassing indirectly, by inducing a particular conformation of the ribosomal subunits and PTC. Hence we presumed that the ribosome itself, in particular the conformation of the decoding center, is responsible for the temperature sensitivity of bypassing. The A site of the ribosome serves as a platform for the estimated interactions between the A-site SL and the tip of EF-G domain IV (Zhou, et al., 2013), which prompted us to test how mutations in the ribosomal decoding site and in EF-G affect the temperature dependence of bypassing. Ribosomal protein $\mathrm{S} 12$ is located at the subunit interface where it is poised to play critical roles in interacting with the tRNA substrates and the large subunit (Cukras et al., 2003). We mutated two amino acid residues in r-protein S12, that are oriented towards the mRNA in the decoding center (Fig. 16). 
Results

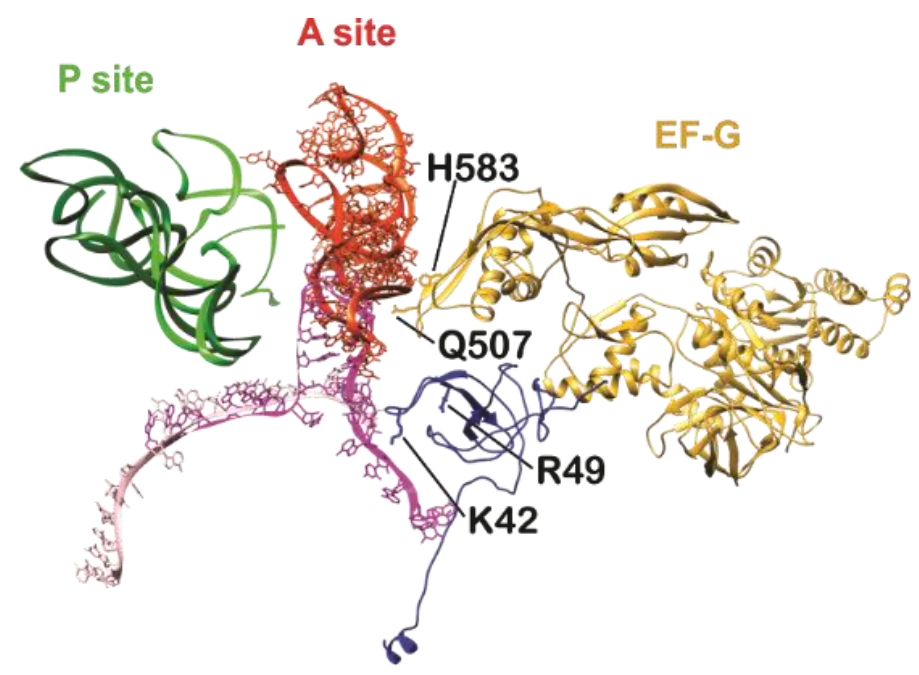

Figure 16. Close-up of the decoding region of the small ribosomal subunit

The structure of a pretranslocation complex with tRNAs in the A and P sites (the tRNAs are red and dark green, respectively) and EF-G (yellow) is superimposed with the structure of the take-off complex with the P-site peptidyl-tRNA (light green) and the SL in the A-site (purple). Positions of point mutations in EF-G and $\mathrm{S} 12$ (dark blue) are indicated. The image was produced by Bee-Zen Peng from structures with Protein Data Bank (PDB) accessions 4V7D and 5NP6 (Brilot et al., 2013; Agirrezabala et al., 2017).

To begin with, we verified that the $\mathrm{S} 12$ mutations do not affect the reactivity of the ribosomes. We used the puromycin (Pmn) time-resolved assay to assess the ability of the mutant ribosomes to perform translocation and compared it to the WT ribosomes (Fig. $17 \mathrm{~A}-\mathrm{B})$. Pmn is an antibiotic that binds to the ribosome from the vacant $A$ site and causes a transpeptidation reaction in a way, that a nascent chain becomes attached to the Pmn itself and the further translation is blocked (Pestka, 1971). PRE complex has low Pmn activity because after accommodation of an incoming A site tRNA both and immediate transpeptidation reaction $A$ and $P$ sites are pre-occupied by peptidyl- and deacylatedtRNAs respectively, making the interaction with the antibiotic impossible. On the contrary, after the translocation is promoted by EF-G (in a POST complex), the peptidyl-tRNA is moved to the $P$ site and is able to interact with Pmn. The rates of tRNA translocation and Pmn reaction are comparable, which allowed us to evaluate the ribosome fraction able to undergo translocation (Rodnina et al., 1997). First, the PRE-complex was formed on a short mRNA (the coding sequence is a dipeptide Met-Phe) such that the A site was occupied with $f\left[{ }^{3} \mathrm{H}\right]$ Met- $\left[{ }^{14} \mathrm{C}\right] \mathrm{Phe}-\mathrm{tRNA}{ }^{\text {Phe }}$ and the $\mathrm{P}$ site was filled with deacylated 
tRNA $^{\mathrm{fMet}}$. Then the purified PRE-complex was incubated with EF-G and mixed with Pmn. The peptide products for each time point were extracted by the ethyl acetate and quantified by ${ }^{3} \mathrm{H}$ and ${ }^{14} \mathrm{C}$ counting. In the case of complete translocation, the tripeptide $\mathrm{f}\left[{ }^{3} \mathrm{H}\right]$ Met- $\left[{ }^{14} \mathrm{C}\right]$ Phe-Pmn was produced; otherwise, the dipeptide $\mathrm{f}\left[{ }^{3} \mathrm{H}\right]$ Met- $\left[{ }^{14} \mathrm{C}\right]$ Phe was detected. Translocation was measured by the ratio of synthesized peptides; $50-65 \%$ of the S12 mutant and WT ribosome complexes were active in EF-G-promoted translocation (Fig. 17A). We also performed the Pmn assay in the absence of EF-G to ascertain, that mutations in $\mathrm{S} 12$ do not cause elevated spontaneous translocation. As a result, the tendency to undergo translocation with EF-G was the same for the mutant and WT ribosomes (7-10\%) (Fig. 17B). We also measured the rate of mRNA translocation using a short 3' fluorescence-labeled (Alexa405) mRNA; the rapid kinetics assay is based on the decrease of fluorescence intensity as the mRNA moves towards the ribosome after the PRE-complex is mixed with EF-G (Holtkamp et al., 2014; Belardinelli et al., 2016) (Fig. 17C). The rate of mRNA translocation was unaltered for $\mathrm{S} 12$ mutant and WT ribosome complexes. Besides, we estimated the stability of peptidyl-tRNA binding to the mutant ribosomes to exclude its spontaneous release caused by $S 12$ mutations (Fig. 17D). We prepared the PRE complexes with $f\left[{ }^{3} \mathrm{H}\right]$ Met- $\left[{ }^{14} \mathrm{C}\right]$ Phe-tRNA ${ }^{\text {Phe }}$ in the $A$ site and deacylated tRNA ${ }^{\mathrm{fMet}}$ in the $\mathrm{P}$ site and incubated them at $37^{\circ} \mathrm{C}$. tRNA binding was assayed by nitrocellulose filtration of the complexes, and the amount of retained tRNA was quantified by ${ }^{3} \mathrm{H}$ and ${ }^{14} \mathrm{C}$ counting. 
A

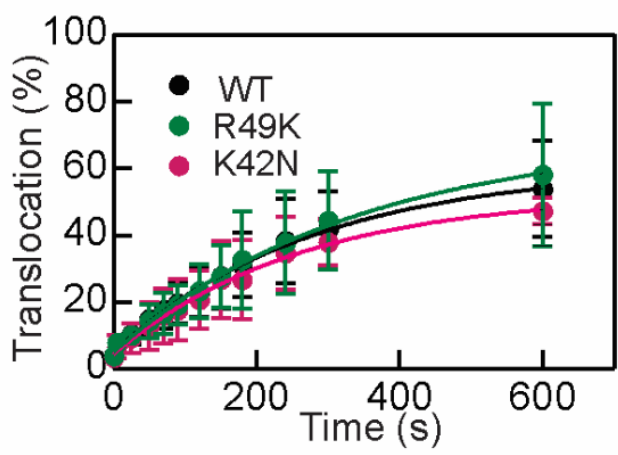

C

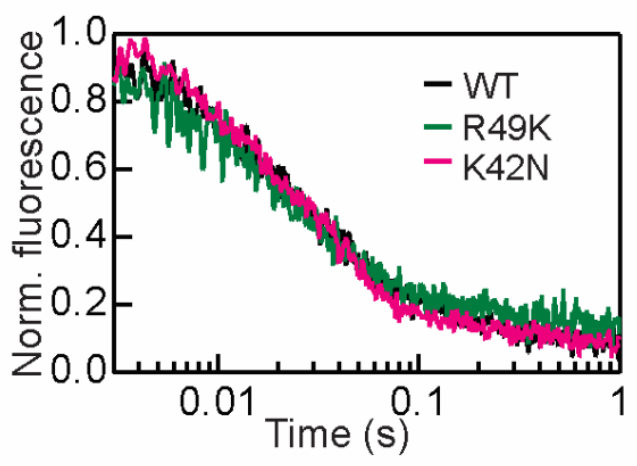

B

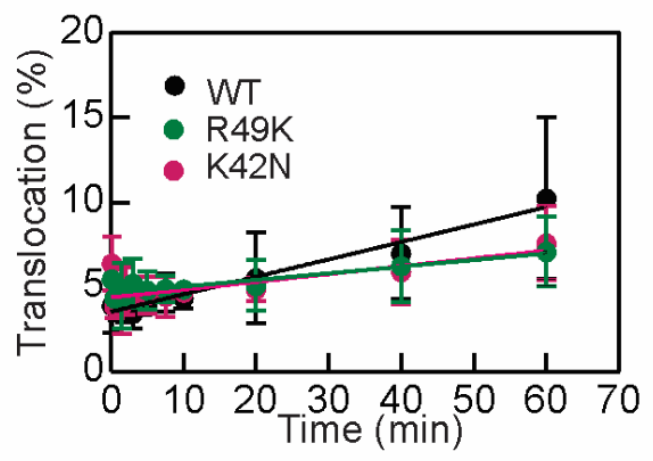

D

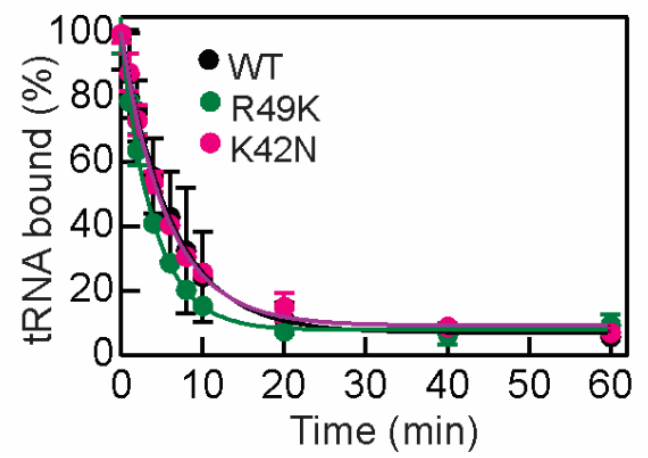

Figure 17. Functional characterization of the ribosomes with mutations in $\mathrm{S} 12$ in comparison with the WT ribosomes

(A) The time-resolved Pmn assay with EF-G added. Error bars show standard deviation (SD) obtained from three independent experiments. (B) The time-resolved Pmn assay without EF-G added (spontaneous translocation). Error bars show standard deviation (SD) obtained from three independent experiments. (C) Single round translocation measured in a stopped-flow apparatus by monitoring the fluorescence change of a fluorescence label (Alexa 405) attached to the 3' end of the mRNA. Traces are the averages from six replicates. The rate of mRNA translocation evaluated by exponential fitting using TableCurve software was $\sim 30 \mathrm{~s}^{-1}$ for both $\mathrm{S} 12$ mutants and WT ribosomes (D) The stability of the peptidyl-tRNA binding to the ribosome. The dissociation rate was $\sim 4 \times 10^{-3} \mathrm{~s}^{-1}$ for both $\mathrm{S} 12$ mutants and WT ribosomes. Error bars show standard deviation (SD) obtained from three replicates. The experiments were performed by Bee-Zen Peng.

As a result, the ability of the ribosomes with $\mathrm{S} 12$ mutations to translocate was unaffected in comparison to WT ribosomes, in addition to unaltered mRNA translocation 
Results

rate and their ability to retain the $\mathrm{P}$ site tRNA. Therefore, the in vitro translation on the long gene 60 mRNA could be performed (Fig. 18A-D).

A

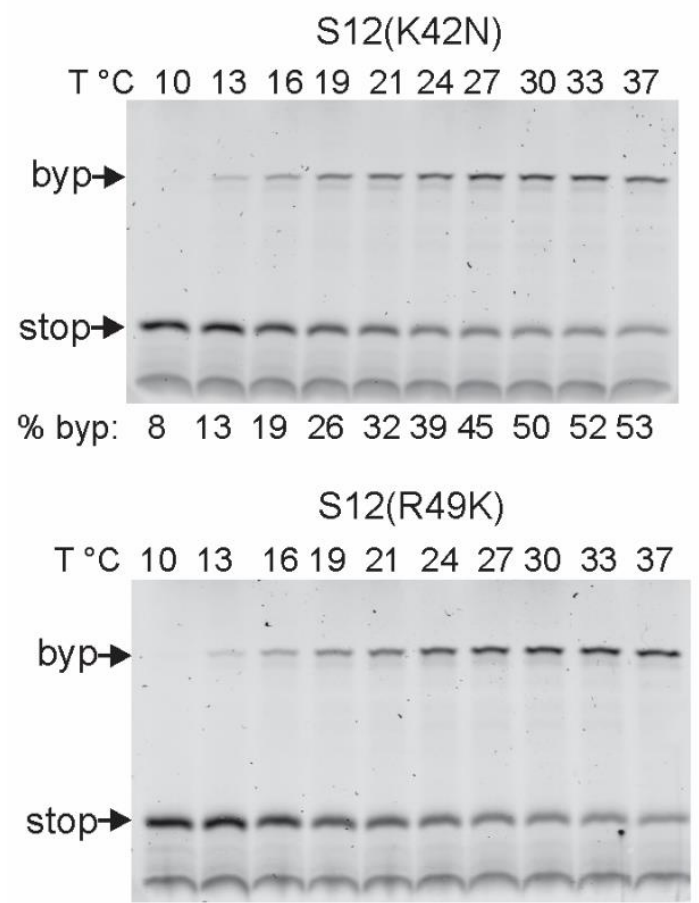

\% byp: $4 \quad 81421263442475052$
B

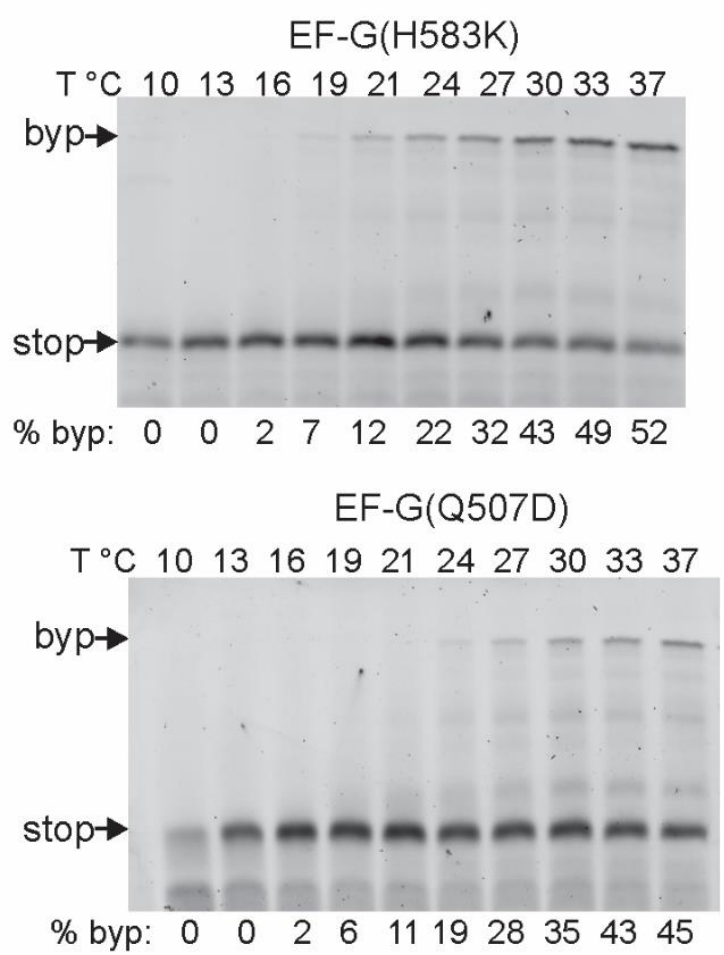

$\mathrm{D}$

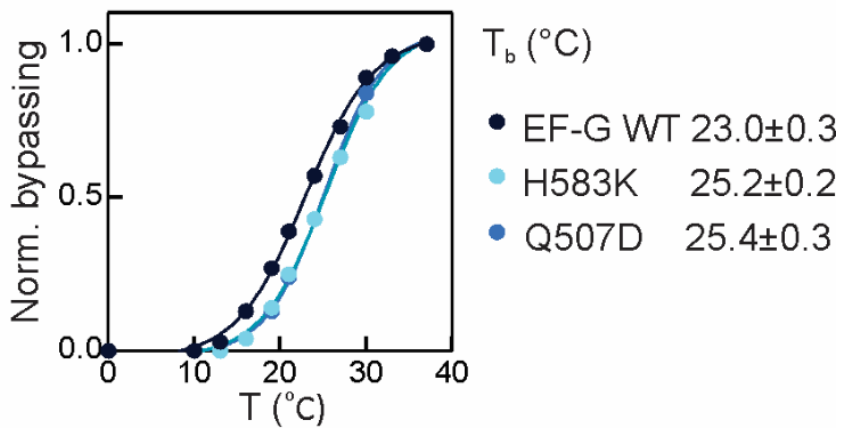

Figure 18. Bypassing of ribosomes carrying mutations in S12 and EF-G with mutations in the domain IV (A) Bypassing at different temperatures for the $\mathrm{S} 12$ mutants. (B) Bypassing at different temperatures for the EF-G mutants. The byp and stop products are indicated with arrows, the incubation temperatures are indicated above the gels. Example of temperature dependence of bypassing for the S12 (C) and EF-G (D) mutants; the bypassing efficiency was normalized for the maximum efficiency at $37^{\circ} \mathrm{C} . \mathrm{T}_{\mathrm{b}}$ is the temperature at which bypassing is half-maximal. $T_{b}$ represented by an average value and a standard deviation (SD) obtained from two replicates. Curves are fit according to the sigmoid model. The S12 and EF-G mutants were provided by Michael Pearson and Bee-Zen Peng respectively. 
Results

Mutations in $\mathrm{S} 12$ shifted the $\mathrm{T}_{b}$ value towards lower temperatures (Fig. $18 \mathrm{~A}, \mathrm{C}$ ); thus, the ribosomes with relaxed phenotype were more prone to start sliding at less favorable temperature conditions than the WT ribosomes. In contrast to $S 12$ mutations, the EF-G variants with mutations H583K or Q507D shifted the $\mathrm{Tb}_{\mathrm{b}}$ towards higher values (Fig. 18B, D).

When combined, S12 and EF-G domain IV mutations were partially compensating each other (Fig. 19A-D). Mutations in domain IV suppressed the relaxed phenotype of the S12 mutants, so we observed $T_{b}$ values approximated to $T_{b}$ for non-mutated components. These data suggest, that bypassing is sensitive to temperature-dependent conformational dynamics of the decoding center, with r-protein S12 restricting and EF-G promoting bypassing (Fig. 20). 
A

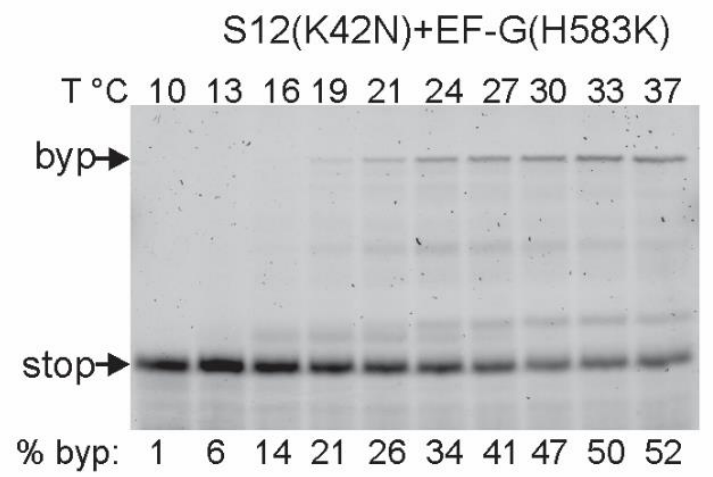

S12(K42N)+EF-G(Q507D)

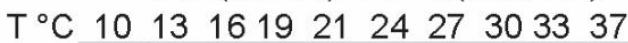

byp $\rightarrow$

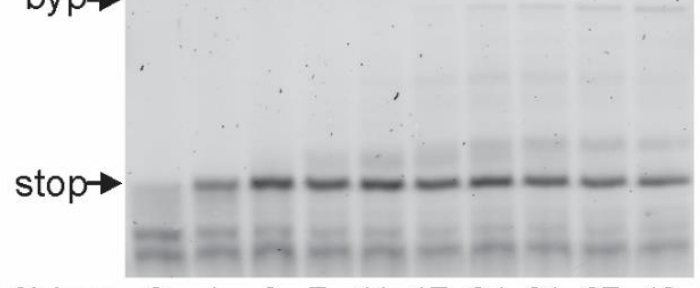

$\begin{array}{lllllllllll}\text { \% byp: } & 0 & 1 & 3 & 7 & 11 & 17 & 24 & 31 & 37 & 40\end{array}$

C

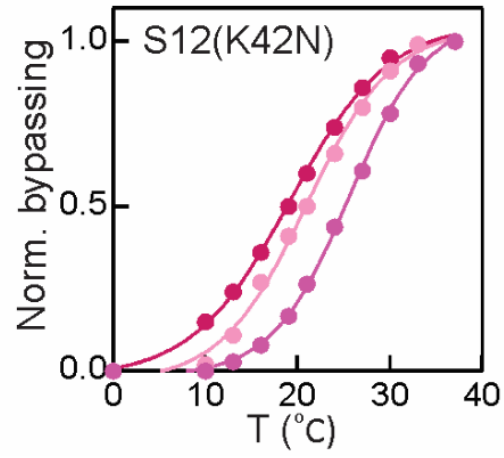

B
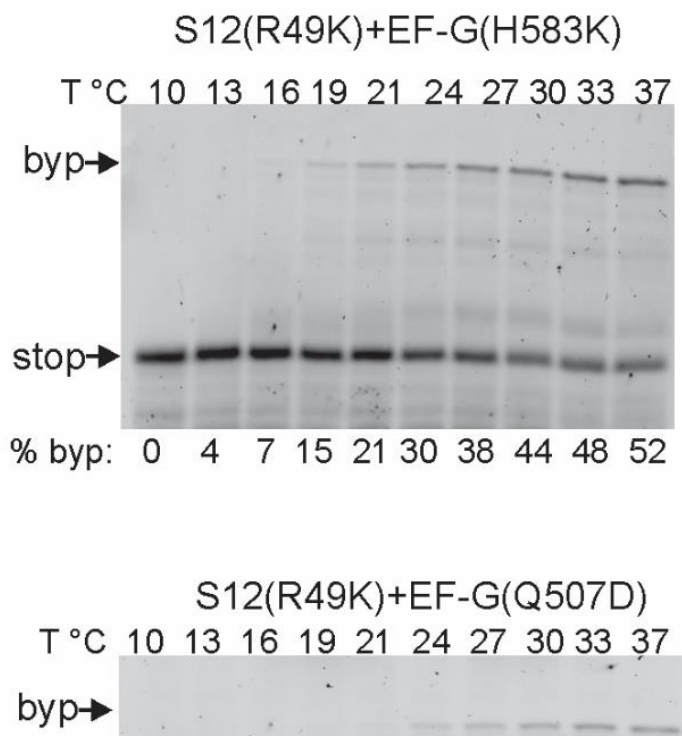

stop $\rightarrow$

\% byp: $\begin{array}{llllllllll}0 & 3 & 4 & 9 & 13 & 20 & 28 & 34 & 40 & 44\end{array}$

Figure 19. Bypassing of ribosomes carrying mutations in $S 12$ combined with EF-G with mutations in the domain IV

(A) Bypassing at different temperatures for the $S 12(\mathrm{~K} 42 \mathrm{~N})$ mutant performed with domain IV EF-G mutants.

(B) Bypassing at different temperatures for the S12 (R49K) mutant performed with domain IV EF-G mutants. The byp and stop products are indicated with arrows, the incubation temperatures are indicated above the gels. Example of the temperature dependence of bypassing for the S12 (K42N) (C) and S12 (R49K) (D) mutants combined with various types of EF-G; the bypassing efficiency was normalized for the maximum efficiency at $37^{\circ} \mathrm{C}$. $T_{b}$ is the temperature at which bypassing is half-maximal. $T_{b}$ represented by an average 
Results

value and a standard deviation (SD) obtained from two replicates. Curves are fit according to the sigmoid model. The S12 and EF-G mutants were provided by Michael Pearson and Bee-Zen Peng respectively.

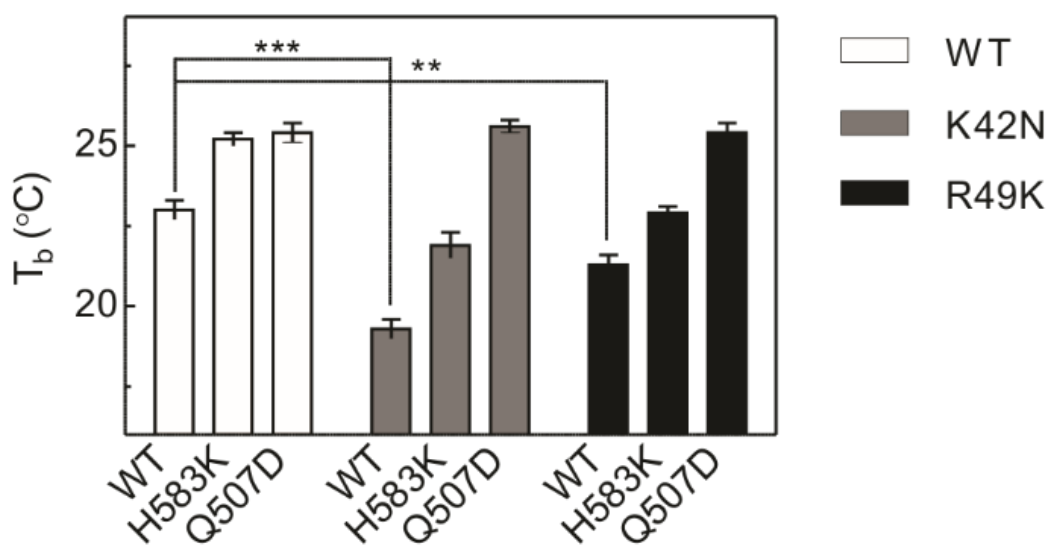

Figure 20. Summary of $T_{b}$ for the combinations of WT and mutant $S 12$ and EF-G Error bars show standard deviation (SD) obtained from three replicates. ${ }^{* *} \mathrm{P}<0.005$ and ${ }^{* * *} \mathrm{P}<0.0005$ by Student's two-tailed unpaired t-test.

\subsection{Dynamics of ribosomal subunits during bypassing initiation and sliding}

In the cryo-EM structure of the ribosome stalled at the take-off site at low temperature, the ribosome adopts an unusual rolled conformation that differs from the classical nonrotated and the rotated states sampled during regular translation (Agirrezabala et al., 2017). To understand the conformational dynamics of the ribosome upon EF-G triggered initiation and during sliding along the non-coding gap, we utilized an established smFRET assay (Ermolenko et al., 2007; Cornish at al., 2008; Sharma et al., 2016; Adio et al., 2018).

FRET causes a decrease in donor fluorescence and a concomitant increase of acceptor fluorescence. The SSU and LSU ribosomal subunits were labeled with the fluorescent dyes Cy5 (acceptor) and Cy3 (donor), respectively and used to form take-off complexes stalled at $10{ }^{\circ} \mathrm{C}$. The ribosome complexes needed to be immobilized on the slide surface, so the system could be investigated for a longer time scale compared to freely diffusing molecules. For stable immobilization we used neutravidin-biotin linkage; a biotinylated DNA primer was annealed to 5' end of gene 60 mRNA and the ribosome 
complexes were attached to a slide coated with neutravidin (Roy et al., 2008; Shebl et al., 2014). The S6-Cy5 and L9-Cy3 FRET pair has been extensively characterized in smFRET experiments; it reports on the formation of the non-rotated or the rotated state of the ribosome (Cornish et al., 2008; Sharma et al., 2016; Adio et al., 2018). Rotation of the subunits relative to each other results in a FRET change between S6-labeled SSU and L9-labeled LSU. The CCW rotation of the SSU relative to the LSU moves the fluorophores apart from each other resulting in a low (0.5) FRET efficiency (rotated state) in and the backward CW rotation of the SSU brings the labels closer together resulting in a high (0.7) FRET efficiency (non-rotated state).

To verify that labeling does not affect the ability of the ribosomes to bypass, we first performed in vitro translation of gene 60 mRNA with non-labeled and labeled subunits and compared the bypassing efficiencies. The bypassing efficiency for labeled subunits (about 40\%) was comparable to that of the non-labeled ribosomes (Fig. 21), therefore we proceeded further with the stalled take-off complex formation and purification.

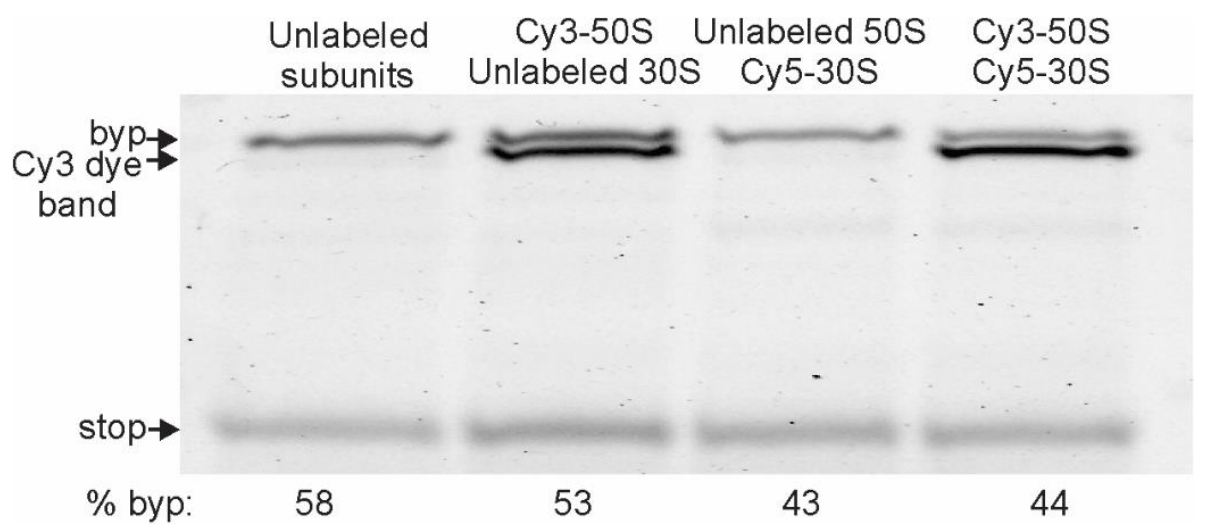

Figure 21. Bypassing activity of fluorescence-labeled ribosomal subunits

Labels are indicated above the gel. The byp and stop products are indicated with arrows. The Cy3- labeled r-protein L9 is visible on the gel, as it is excited by the same laser wavelength as BODIPY FL dye.

First, we analyzed the stalled take-off complexes similar to those utilized in cryo-EM studies, i.e. in the rolled state (Agirrezabala et al., 2017). We observed, that the majority of the stalled take-off complexes at low temperatures $\left(4-10^{\circ} \mathrm{C}\right)$ were sampled in the nonrotated state (FRET 0.7) (Fig. 22A). This can be explained by the similarity of the distances between the fluorescent reporters in rolled and non-rotated states. Typically, the preparation of cryo-EM complexes is performed at low temperatures to decrease 
Results

thermal fluctuations of the ribosome which facilitates visualization of the most populated state. It had been shown that a temperature change during complex preparation affects the distribution between sampled states. A PRE ribosome complex was detected to be predominantly in the non-rotated state at $4{ }^{\circ} \mathrm{C}$, whereas elevated temperatures caused a bimodal distribution of rotated and non-rotated states (Fischer et al., 2010). Because the temperature is critical for bypassing, we also wanted to measure subunit rotation at elevated temperatures.

A

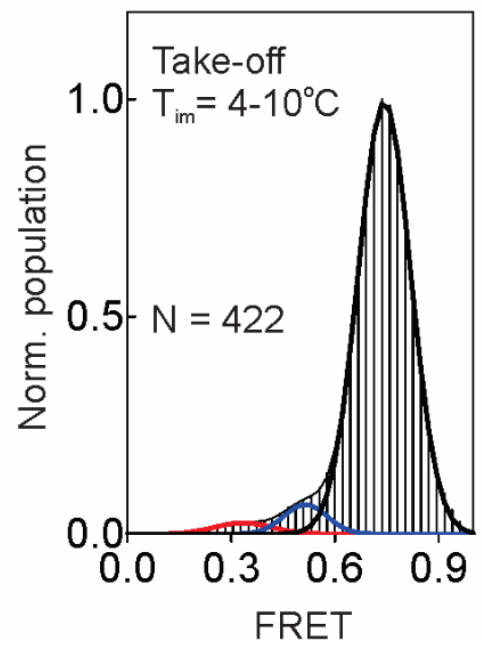

$B$

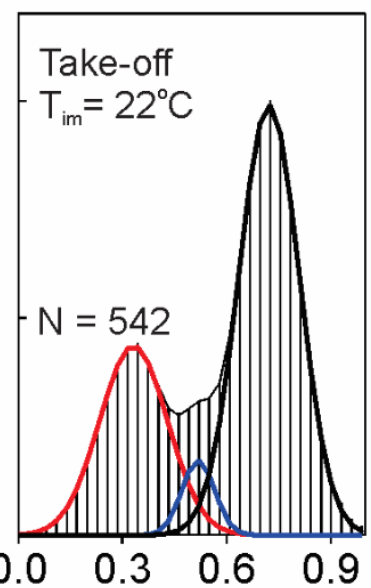

FRET
C

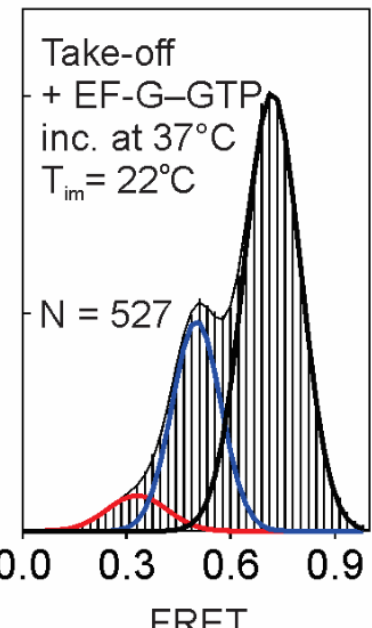

FRET

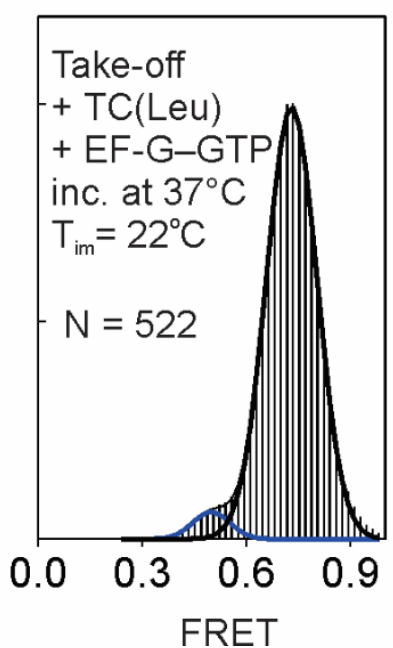

FRET

Figure 22. Ribosomal conformations during bypassing on gene 60 mRNA visualized by smFRET (A) FRET distribution histogram for the purified take-off complex at the low temperature. The main ribosome populations with FRET efficiencies of $0.3,0.5$ and 07 are indicated with red, blue and black lines, respectively. The number of traces $(N)$ and the imaging temperature $\left(T_{i m}\right)$ are indicated in each panel. $(B)$ FRET distribution histogram for the purified take-off complex at the elevated temperature. (C) Ribosome conformation of the landing complex obtained by incubation of the take-off complex at $37^{\circ} \mathrm{C}$ with EF-GGTP added. (D) Ribosome conformation after the addition of EF-G-GTP and EF-Tu-GTP-Leu-tRNA ${ }^{\text {Leu }_{U U A}}$ at $37^{\circ} \mathrm{C}$. smFRET experiments were performed and analyzed by Dr. Tamara Senyushkina.

Upon incubation of the take-off complex at $22^{\circ} \mathrm{C}$ in the absence of additional components, a significant ribosome population with the lowest FRET efficiency of 0.3 was distinguished (Fig. 22B), indicating a further increase in the distance between the two fluorescent dyes. This previously reported lower FRET state represents a higher degree of subunit rotation and corresponds to the hyper-rotated state formation (Qin et al., 2014). After incubation of the take-off complex with EF-G-GTP at $37^{\circ} \mathrm{C}$ (conditions previously 
used for efficient bypassing initiation in vitro) we observed the ribosomes returning to the rotated state (Fig. 22C). Addition of EF-Tu-GTP-Leu-tRNA ${ }^{\text {Leu, }}$ which allows for Leu incorporation into the nascent peptide chain and a round of canonical translocation after landing, brought the ribosomes to the non-rotated state (Fig. 22D).

To determine which element of the gene $60 \mathrm{mRNA}$ contributes to the formation of the low 0.3 FRET state, we studied take-off complexes formed with mutated mRNAs at $4^{\circ} \mathrm{C}$ and $22^{\circ} \mathrm{C}$. We observed, that the majority of complexes with substitution in the essential KKYK motif of the nascent peptide remained predominantly in the non-rotated state both at low (Fig. 23A) and elevated temperatures (Fig. 23B), i.e. the formation of the hyperrotated state was inhibited. In the case of take-off complexes lacking the A-site SL (Fig. $23 \mathrm{C}$ ), only negligible amounts of hyper-rotated state ribosomes were detected at $22^{\circ} \mathrm{C}$. After incubation of the take-off complex with EF-G-GTP at $37^{\circ} \mathrm{C}$ we also observed the loss of the small hyper-rotated fraction (Fig. 23F). Therefore, both the nascent peptide interactions in the exit tunnel and the A-site SL structure are essential for the formation of the hyper-rotated state. We suggest, therefore, that a dynamic transition from hyperrotated state to the rotated state upon EF-G interaction with the A-site SL is a hallmark of a successful bypassing event. 
A

B
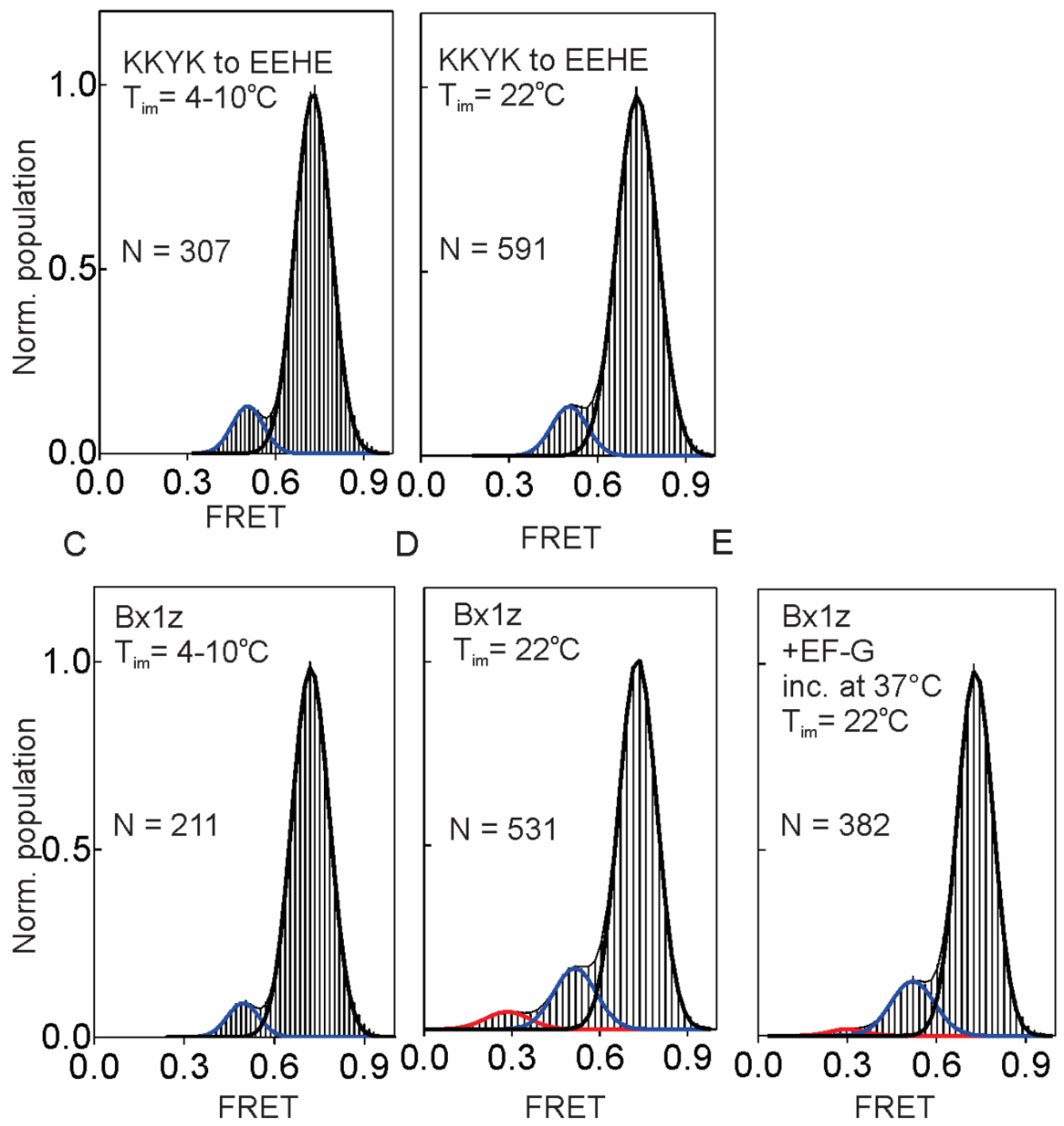

Figure 23. Ribosomal conformations during bypassing on different gene 60 mRNA constructs visualized by SMFRET

(A) FRET distribution histogram for the purified take-off complex with substituted KKYK motif at the low temperature. The main ribosome populations with FRET efficiencies of $0.3,0.5$ and 0.7 are indicated with red, blue and black lines respectively. The number of traces $(\mathrm{N})$ and the imaging temperature $\left(\mathrm{T}_{\mathrm{im}}\right)$ are indicated in each panel. (B) FRET distribution histogram for the purified take-off complex with substituted KKYK motif at the elevated temperature. (C) FRET distribution histogram for the purified take-off complex with partially unfolded A-site SL at the low temperature. (D) FRET distribution histogram for the purified take-off complex with partially unfolded $A$-site $S L$ at the elevated temperature. (F) Ribosome conformation or the purified take-off complex with partially unfolded A-site SL after the addition of EF-G-GTP at $37^{\circ} \mathrm{C}$. smFRET experiments were performed and analyzed by Dr. Tamara Senyushkina. 
We should note that due to the complexity of purification procedures, a pool of residual non-active stalled ribosomes could not be separated from the ribosomes active in translation and bypassing. As was previously shown, the ribosome adopts the classical non-rotated state as it becomes fully assembled during initiation (Marshall et al., 2009). The fluorescence-labeled ribosomes which reside at the start codon are sampled in the non-rotated state, therefore they contribute to the non-rotated ribosomal population and cause heterogeneity in take-off complex preparations. We presumed, that the complexes active in bypassing can change the conformation from the non-rotated to rotated or hyperrotated state. According to the distribution of FRET states, about $40 \%$ of complexes were showing distinct dynamics under the conditions of the smFRET experiment (cf. Fig. 22AB). This assumption is in good agreement with biochemical experiments ( $40 \%$ bypassing efficiency for labeled ribosomes) (cf. Fig. 21). To simplify the final comparisons between different experimental conditions and to estimate the FRET signal change, we normalized the data to show only the potentially active ribosomal complexes expected (Fig. 24).

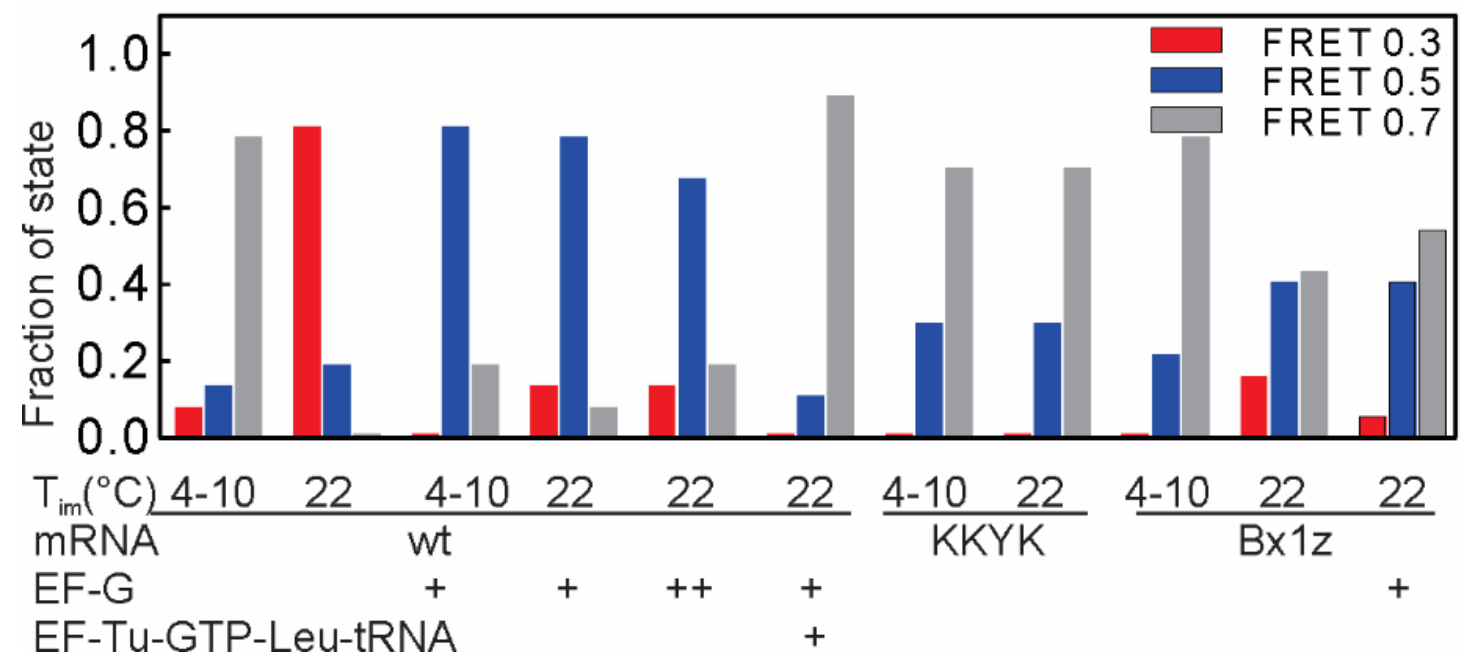

Figure 24. Distribution of hyper-rotated, rotated, and non-rotated ribosomal populations in different complexes normalized to the activity of the take-off complex

Ribosomes trapped by the SecM stalling motif had been shown to adopt a rolled conformation similar to that of the take-off complexes. The stalling is achieved by a different mechanism: essential Arg and Pro residues of 17-amino-acid SecM stalling 
Results

sequence interact with the peptide tunnel and cause PTC inactivation (Nakatogawa \& Ito, 2002; Zhang et al., 2015). Moreover, recent smFRET studies also reported similarities of the SecM and gene 60 translation, namely increased lifetimes of rotated and non-rotated states as the ribosomes approach the SecM stalling sequence and take-off site respectively (Tsai et al., 2014; Chen at al., 2015). We tested whether SecM complexes also undergo temperature-related conformational changes during stalling, i.e. proceed through the hyper-rotated state formation.

We formed SecM stalled complexes with the labeled subunits, immobilized them on a slide surface using biotinylated DNA primer specific for the SecM mRNA sequence and monitored the FRET signal at different temperatures. At low temperatures, the stalled SecM complexes resided in the rotated and non-rotated states (Fig. 25A). When the temperature was increased to $22^{\circ} \mathrm{C}$ a ribosome fraction also adopted the 0.3 FRET state which we identified as the hyper-rotated state for the bypassing complexes (Fig. 25B). In this way we identified the hyper-rotated state formation for two functionally unrelated types of stalled ribosomal complexes.
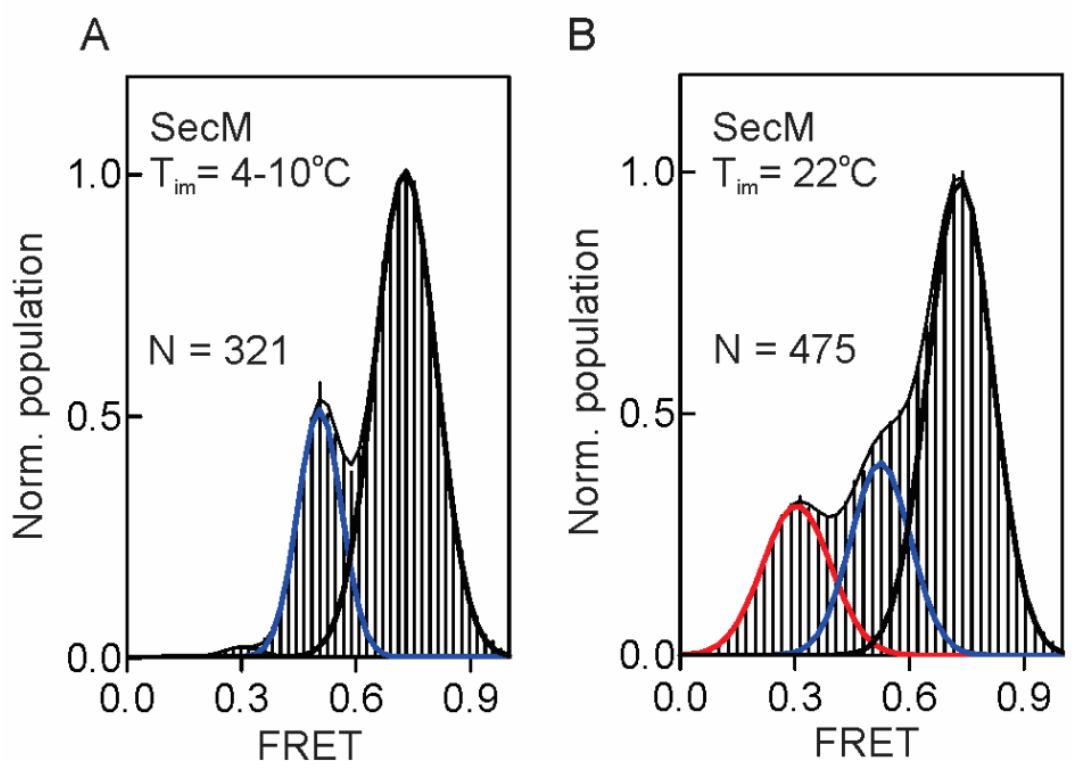

Figure 25. Ribosomal conformations during SecM stalling visualized by smFRET

(A) FRET distribution histograms for the SecM-stalled ribosome complexes at low temperatures. (B) FRET distribution histograms for the SecM-stalled ribosome complexes at elevated temperatures. The main ribosome populations with FRET efficiencies of $0.3,0.5$, and 0.7 are indicated with red, blue and black lines respectively. The number of traces $(\mathrm{N})$ and the imaging temperature $\left(\mathrm{T}_{\mathrm{im}}\right)$ are indicated in each panel. smFRET experiments were performed and analyzed by Dr. Tamara Senyushkina. 


\subsection{The effect of ribosomal protein L9 on programmed bypassing in vitro}

Ribosomal protein L9 has been suggested to prevent forward slipping of the ribosome during translation and to serve as an indirect regulator of translational bypassing (Wills et al., 2008). As was shown in vivo (Herr et al., 2001), ribosomal bypassing over a stop codon (stop hopping), increases 8-fold for ribosomes lacking the L9 protein. The mechanism by which L9 contributes to these processes is unclear; it does not affect the structure of the peptidyl transferase nor the one of the decoding center of the ribosome. As all available data on bypassing was obtained in vivo and could reflect multiple cellular processes, we sought to investigate the role of L9 in programmed gene 60 bypassing in vitro. We studied the contribution of protein L9 on programmed bypassing in vitro in several aspects. We used $\Delta \mathrm{L} 9$ ribosomes and tested the effect of the deletion on WT gene $60 \mathrm{mRNA}$ at different temperatures and the effect of mutations in essential mRNA signals. Because L9 was presumed to disrupt ribosomal coupling within a polysome array and thereby facilitating independent slippage (Smith, et al., 2019), we tested the potential regulatory activity of $L 9$ under conditions of polysome formation.

$\Delta \mathrm{L} 9$ ribosomes were as active as $\mathrm{WT}$ ribosomes at forming initiation complex, close to $100 \%$ (Fig. 26). When we investigated the temperature dependence of gene 60 translation with $\mathrm{WT}$ and $\Delta \mathrm{L} 9$ ribosomes, we found that both translation and bypassing efficiencies were significantly lower for $\Delta L 9$ ribosomes (Fig. 27A-B), that means that deletion of protein L9 negatively affects ribosomal activity in translation in vitro. Our goal was to detect a potential shift in the behavior of the WT and $\triangle \mathrm{L} 9$ ribosomes during gene 60 mRNA translation. Therefore we further compared the bypassing efficiencies normalized for the maximum efficiency at $37^{\circ} \mathrm{C}$ on WT mRNA for both WT and $\triangle \mathrm{L} 9$ ribosomes. Changing the translation incubation temperature did not reveal a pronounced change in the $T_{b \Delta L 9}$ value $\left(22.2 \pm 0.3^{\circ} \mathrm{C}\right)$ compared to the value for WT ribosomes $\left(21.8 \pm 0.3^{\circ} \mathrm{C}\right)$ (Fig. 27C). 
Results

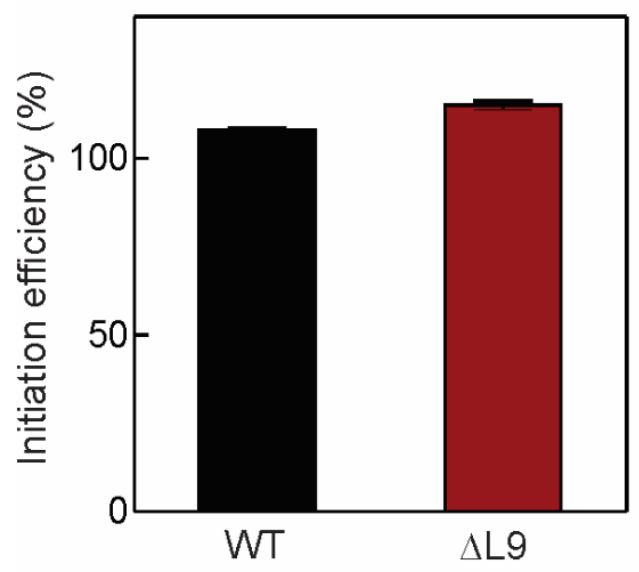

Figure 26. Initiation efficiency for intact (WT) and mutant $(\Delta \mathrm{L} 9)$ ribosomes on the gene $60 \mathrm{mRNA}$. Error bars show standard deviation (SD) obtained from two replicates.

A

B

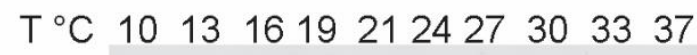

byp $\rightarrow$

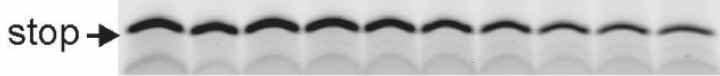

$\mathrm{T}^{\circ} \mathrm{C} 101316192124273033 \quad 37$

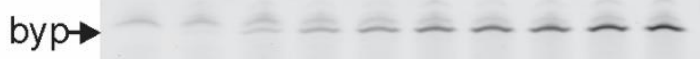

stop $\rightarrow$
C

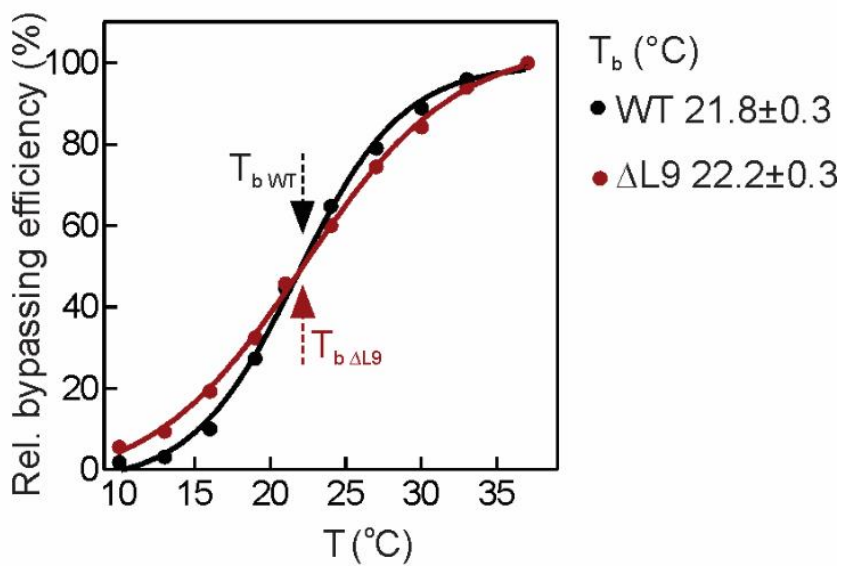

Figure 27. Effect of $L 9$ deletion on the temperature dependence of bypassing

(A) Bypassing at different temperatures for WT and (B) $\Delta \mathrm{L} 9$ ribosomes. The byp and stop products are indicated with arrows, the incubation temperatures are indicated above the gels. (C) Normalized temperature dependence of bypassing for WT and $\Delta L 9$ ribosomes. $T_{b}$ is the temperature at which bypassing is half-maximal. $T_{b}$ is represented by an average value and a standart deviation (SD) obtained from two replicates. Curves are fit according to the sigmoid model.

As a next step, we used our set of A-site SL (cf. Fig. 12), 5'SL (cf. Fig. 14) and the nascent peptide sequence (cf. Fig. 15) mRNA mutants which we had shown to affect bypassing efficiency. For those mutants, we compared the effect on WT and $\Delta \mathrm{L} 9$ ribosomes in our bypassing assay. The dependence of bypassing efficiency on the SL stability was similar 
for both types of ribosomes (Fig. 28A); weakening or stabilizing the A-site SL did not affect the relative bypassing efficiency for the $\Delta \mathrm{L} 9$ ribosomes. Mutations in the 5 'SL also did not bring a significant change in the relative bypassing efficiencies (Fig. 28B). This observation suggests that altering the mRNA bypassing signals do not increase the bypassing efficiency for $\Delta \mathrm{L} 9$ relative to the WT ribosomes.

A

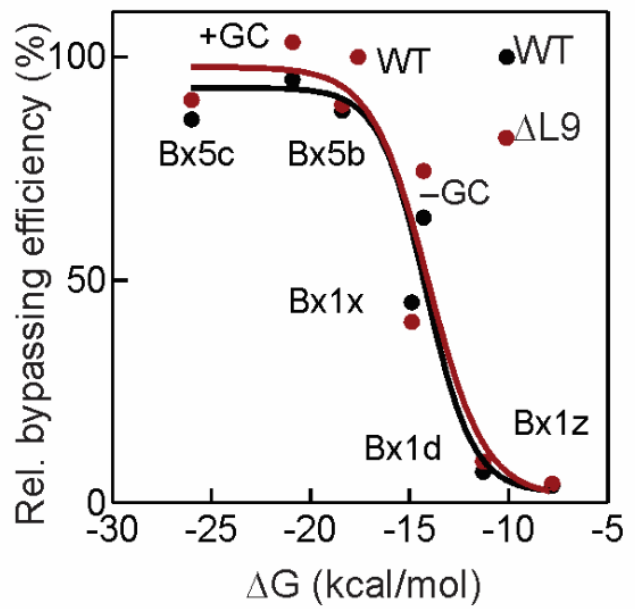

$\mathrm{B}$

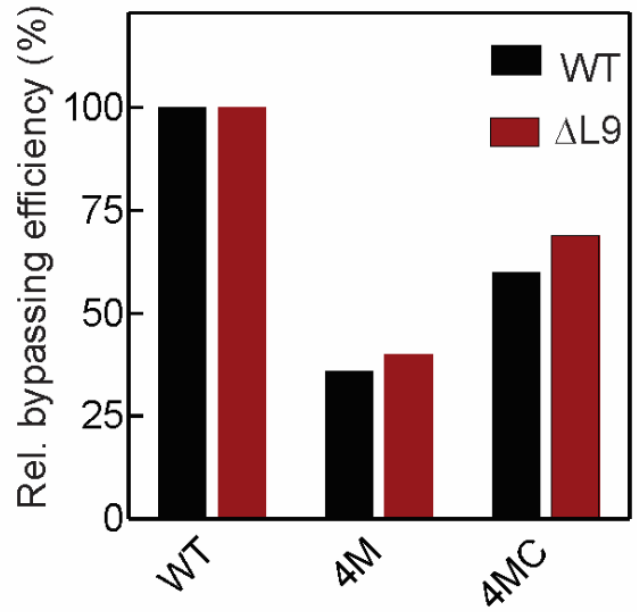

Figure 28. Relative bypassing efficiencies of WT and $\Delta \mathrm{L} 9$ ribosomes on different gene $60 \mathrm{mRNA}$ constructs

(A) The relative bypassing efficiencies are plotted against the predicted folding energies of the A-site SL mutants (cf. Fig. 2.6A). Bypassing efficiencies were normalized to the maximum efficiency at $37^{\circ} \mathrm{C}$ on WT mRNA. Curves fit according to the sigmoid model are shown as continuous lines. (B) Effect of mutations in the $5^{\prime} \mathrm{SL}$ on bypassing efficiency, comparison of $\mathrm{WT}$ and $\Delta \mathrm{L} 9$ ribosomes; the bypassing efficiency was normalized for the maximum efficiency at $37^{\circ} \mathrm{C}$ on WT mRNA.

To test the interaction of the nascent peptide with the exit tunnel in more detail, we used mRNAs with consecutive point mutations of each amino acid in the essential KKYKLQNNVRRSIKSSS ${ }^{14-30}$ motif (Fig. 29A-B). In summary, despite an overall decrease of in vitro translation efficiency upon L9 deletion, the relative bypassing efficiency was similar for both intact and mutant ribosomes for every mutated bypassing signal tested. 
Results

A

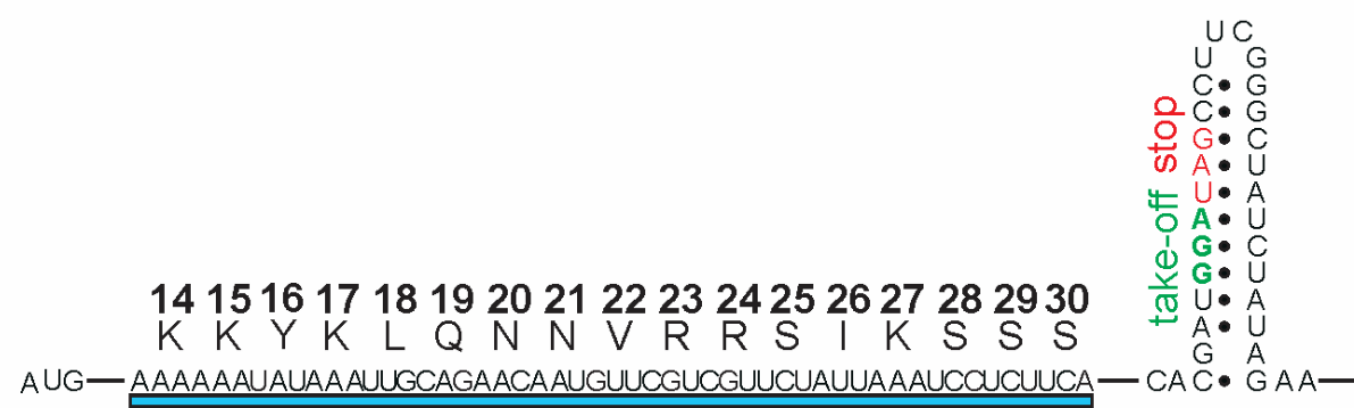

B

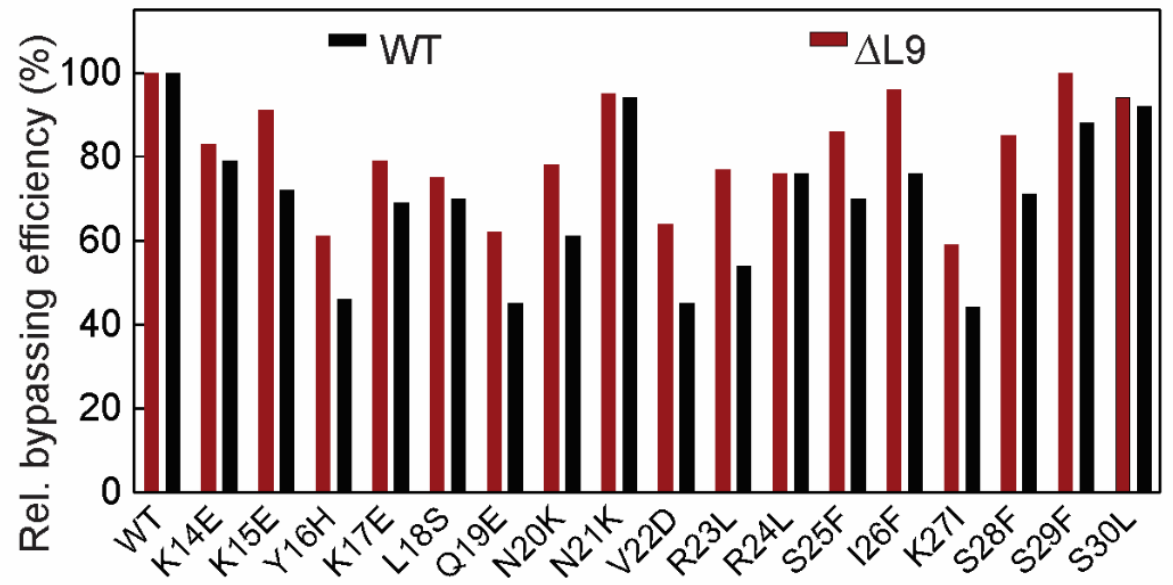

Figure 29. Effect of mutations in the nascent peptide on the bypassing efficiency of WT and $\Delta L 9$ ribosomes

(A) The sequence of gene 60 mRNA upstream the take-off codon. The crucial part of the nascent peptide is underlined; amino acid sequence and positions in the nascent peptide sequence are indicated above the mRNA sequence. (B) Effect of mutations in the nascent peptide on the bypassing efficiency, comparison of WT and $\Delta \mathrm{L} 9$ ribosomes; the bypassing efficiency was normalized for the maximum efficiency at $37^{\circ} \mathrm{C}$ on WT mRNA.

\subsection{The effect of polysome formation on bypassing efficiency}

Next, we asked whether protein L9 has activity within a polysome array. We designed an experimental approach to form polysomes, in which we added initiator tRNA, ribosomal subunits and initiation factors to purified initiation complexes, thereby enabling re-initiation due to the excess of ribosomes over the mRNA. Monosome translation conditions for WT and $\Delta \mathrm{L} 9$ ribosomes (i.e. translation without re-initiation) were used as a control. To better understand the possible L9 effect on polysome formation during translation, we performed time-course experiments with WT and $\Delta \mathrm{L} 9$ ribosomes. In this 
way, we not only evaluated the efficiency of the final full-length product synthesis but were also able to analyze intermediate translation products in a time-resolved manner. We found that the translation, as well as the relative bypassing efficiencies were almost identical for WT and $\Delta \mathrm{L} 9$ ribosomes. But intriguingly, we observed a notable difference between the byp/stop product ratio under mono- and polysome formation conditions for WT ribosomes in vitro (Fig. 30A-D).

The monosome translational time course showed stop product accumulation starting after $7 \mathrm{~s}$ of translation and continued to the moment when the ribosomes reached the take-off site (after $20 \mathrm{~s}$ of translation), where $60 \%$ of them successfully took off, reached the landing site and resumed translation on ORF2 (Fig. 30A). 
Results

A

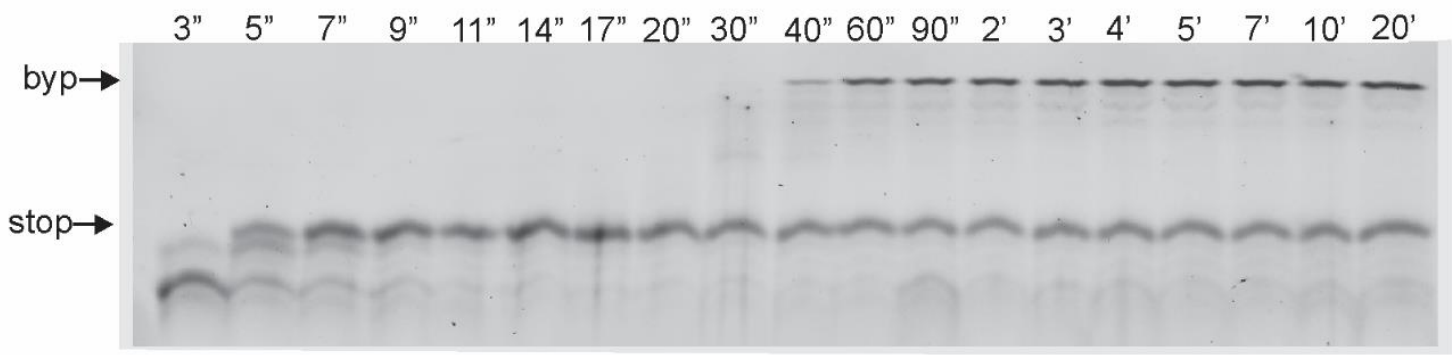

B

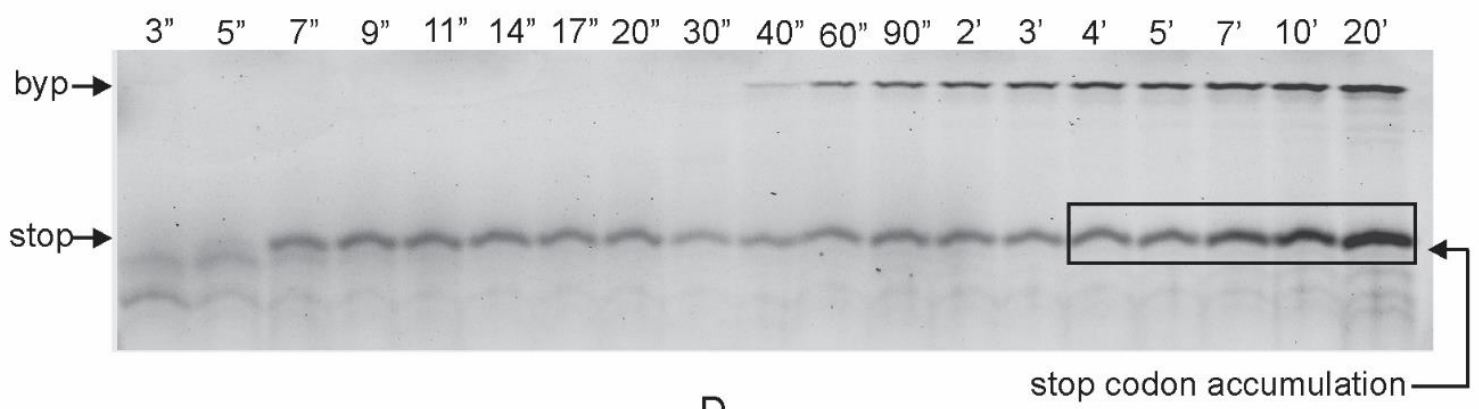

C

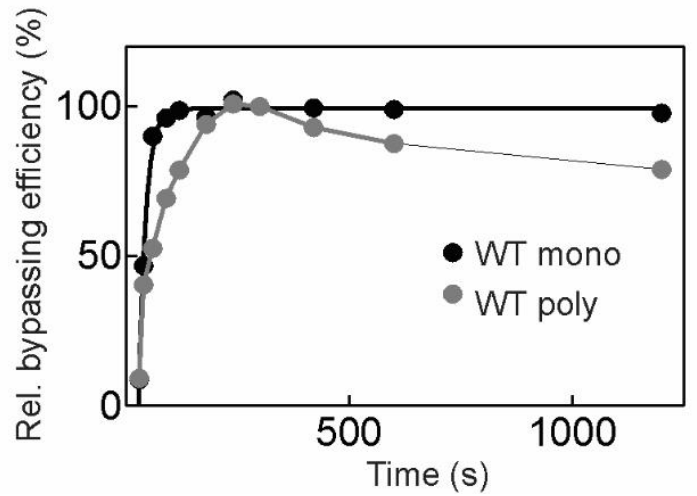

Figure 30. The effect of polysome formation on bypassing efficiency for WT ribosomes

(A) Time courses of stop- and bypassing-product synthesis by monosomes or polysomes (B) formed of WT ribosomes. Translation times ( 3 seconds -20 minutes) and stop codon accumulation process are indicated. (C) The intensity of the stop products produced by monosomes and polysomes in vitro are plotted as a function of translation time. (D) Relative bypassing efficiencies for monosomes and polysomes for WT ribosomes as a function of translation time; the bypassing efficiency was normalized to the maximum efficiency at $37^{\circ} \mathrm{C}$.

In the case of polysomes, the stop product accumulation was continuous during the whole time course of translation (Fig. 30B). We observed that the initial stop product intensity decreased after $20 \mathrm{~s}$ of translation but started to increase steadily again after about $40 \mathrm{~s}$ until the last time point (Fig. 30C). This result indicated that the ribosomes 
completed the re-initiation process and translated to the end of ORF1, but did not bypass successfully, either due to a failure of take-off, of sliding or of landing. This led to a noticeable change in byp/stop products ratio and to a $\sim 20 \%$ decrease of bypassing efficiency under conditions of polysome formation (Fig. 30D). With this experimental setup, we could now test the effect of the protein L9 deletion of bypassing. With the $\Delta L 9$ ribosomes, we did not detect any noticeable change in translational time course under the mono- and polysome formation conditions in comparison to the intact ribosomes (Fig. $31 \mathrm{~A}-\mathrm{B})$. 
Results

A

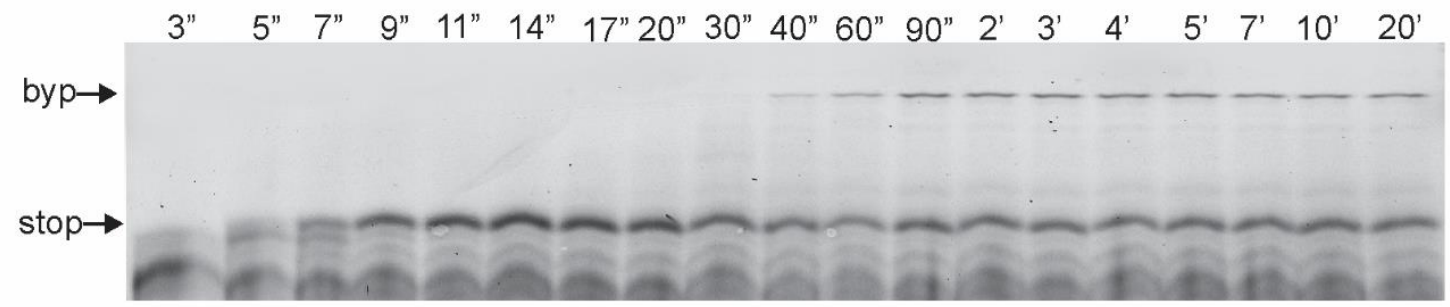

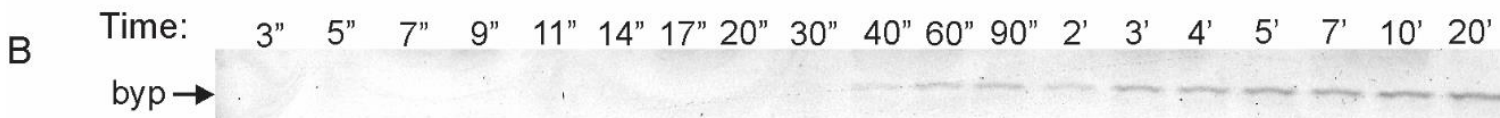
stop $\rightarrow$

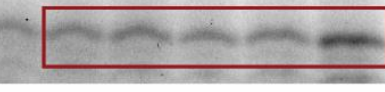

stop codon accumulation

C

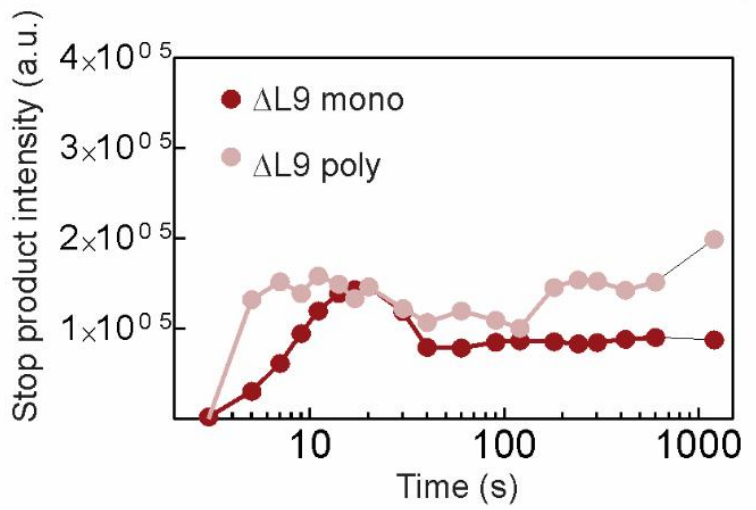

D

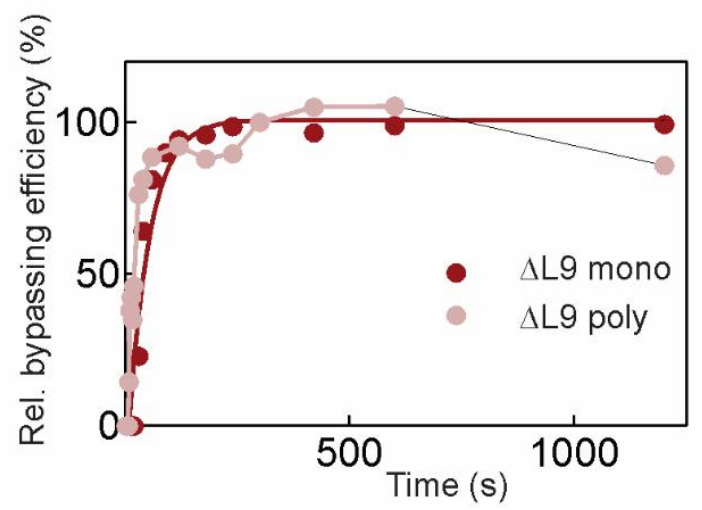

Figure 31. The effect of polysome formation on bypassing efficiency for $\Delta L 9$ ribosomes

Time courses of stop- and bypassing-product synthesis by monosomes (A) or polysomes (B) formed with $\Delta \mathrm{L} 9$ ribosomes. Translation times ( 3 seconds -20 minutes) and stop codon accumulation process are indicated on the gel. (C) The intensity of the stop products produced by monosomes and polysomes in vitro is plotted as a function of translation time. (D) Relative bypassing efficiencies for monosomes and polysomes for $\Delta \mathrm{L} 9$ ribosomes as a function of translation time; the bypassing efficiency was normalized to the maximum efficiency at $37^{\circ} \mathrm{C}$

However, upon polysome formation, we observed the same continuous stop product accumulation as well as a significant difference between the byp/stop product ratio ( 15\%) (Fig. 31C-D). More precise evaluation was hindered due to reduced activity of mutant ribosomes in translation in vitro. In summary, the formation of polysomes on gene 
Results

60 mRNA in vitro reduced the bypassing efficiency with both WT and $\Delta \mathrm{L} 9$ ribosomes, a particular effect of the L9 deletion on bypassing was not observed. 


\section{Discussion}

\subsection{EF-G-induced ribosome sliding along the noncoding mRNA}

In the present study, we show that EF-G promotes sliding of the ribosome along the non-coding gap of the gene 60 mRNA and we suggest how bypassing might be initiated. During the translation of the gene 60 mRNA the ribosome noticeably slows down while translating the last five codons of the ORF1 due to the interactions of the nascent peptide signal with the peptide exit tunnel (Chen et al., 2015). This slowdown upon reaching the take-off site is detected by the increased lifetimes of both non-rotated and rotated states. A similar deceleration of translation was previously reported for nascent peptide-ribosome interactions during SecM stalling (Tsai et al., 2014). Prior to bypassing the ribosome pauses on the last codon of ORF1 which is denoted as the take-off codon; this is observed by the long lifetime of the rotated state. This long-living state during pausing on the takeoff site was suggested to be reminiscent of the non-canonical rotated state detected in the -1PRF studies and characterized by a 10 -fold longer pause in elongation in the case of mRNA recoding by -1PRF (Chen at al., 2014, Chen et al., 2015). Recent studies showed, that during pausing on a slippery sequence during -1PRF the ribosomes adopt the hyper-rotated state induced by the dnaX mRNA hairpin (Qin et al., 2014). During the ribosome pausing on the take-off site, we detect the formation of the non-canonical rotated state (i.e. a state with a higher degree of subunit rotation than in the canonical rotated state), which corresponds to the hyper-rotated intermediate. Most likely the hyperrotated state was not captured in the previous studies of bypassing because of a different FRET pair choice (Chen et al., 2015).

In the cryo-EM structure, ribosomes paused on the take-off site adopt a non-canonical rolled conformation with the PTC in an inactive conformation, the $\alpha$-helical nascent peptide chain formed in the LSU exit tunnel and the short SL formed in the A site (Agirrezabala et al., 2017). A similar rolled conformation was also reported for the ribosomes arrested during translation of the SecM stalling sequence, which is not related to bypassing (Zhang et al., 2015). Our smFRET findings provide the evidence that most likely during gene 60 translation the rolled state observed at low temperature by cryo-EM 
and the hyper-rotated states observed at higher temperatures result from the same stalling signals and reflect transitional conformations of the ribosome, which can rearrange into each other at permissive temperatures. We also corroborate the importance of the nascent peptide signal and the A-site SL for successful bypassing. Our data demonstrate that the hyper-rotated state on the take-off site is part of an unconventional dynamics mode of the ribosome, inherent to paused complexes before resuming translation. In the case of gene 60 translation, the transition from the hyperrotated to the rotated state should be considered a hallmark of bypassing. Moreover, we have observed the non-canonical hyper-rotated state not only in the stalled gene 60 mRNA take-off complexes but also in the complexes stalled at the SecM sequence, which causes ribosome stalling through a different, Pro-dependent mechanism (Zhang et al., 2015). Taken together, this suggests that the hyper-rotated conformation is a characteristic feature of stalled complexes, which needs to be adopted before resuming translation. Both bypassing and SecM stalling depend on particular sequences of the encoded nascent peptide. This underlines the importance of the nascent peptide chain for controlling fundamental steps of translation.

The modifications introduced to disrupt the essential mRNA signals do not affect the temperature dependence of bypassing. Therefore, we suggest it is the ribosome itself with its temperature-driven conformational fluctuations of the decoding site that causes the observed temperature dependence. This is further supported by the effect of mutations in R-protein S12. S12 serves as a control element in the decoding center of the SSU (Cukras et al., 2003) and mutations in S12 were shown to result in a relaxed phenotype with decreased decoding accuracy. These mutations also facilitate bypassing at lower temperatures. This leads us to the conclusion that the temperature-dependent conformational dynamics of the decoding center cause the temperature sensitivity of bypassing with the $r$-protein $\mathrm{S} 12$ serving as a restricting element.

We propose that the initiation of bypassing entails several steps. First, because in our system the ribosome complexes stalled at the take-off site are formed at low temperature, the temperature has to rise to activate the ribosomes. The bypassing signals encoded in 
the gene $60 \mathrm{mRNA}$ activate the ribosome on the take-off site by promoting the formation of the hyper-rotated state. According to the cryo-EM structure (Agirrezabala et al., 2017), the A-site SL occupies the space where the tRNA anticodon domain is located during canonical translocation. Our results show the necessity for the upper part of the A-site SL to have certain folding stability to allow bypassing initiation. We suggest that this is required because of the subsequent interaction of EF-G with the SL. The SL also serves to protect the P-site peptidyl-tRNA from binding of class 1 release factors to the $A$ site, i.e. to exclude premature termination or read-through during pausing on the take-off site. In the second step, the recruited EF-G binds to the hyper-rotated ribosome and interacts with the A-site SL. This interaction might be similar to the reported one with a small tRNA fragment of only $14 \mathrm{nt}$, the anticodon $S L$ in the A site, which is sufficient for EF-Gpromoted tRNA-mRNA translocation (Joseph et al., 1997). During conventional translocation EF-G domain IV is extended towards the SSU A site and comes in direct contact with the anti-codon SL of the A-site tRNA. We suggest that during the take-off phase EF-G domain IV uses the mRNA SL formed the A site as a tRNA mimic, thereby promoting an event designated as pseudo-translocation. Unlike in canonical translocation, in this process no tRNAs are actually moved, i.e. the P site tRNA is not moved to the $E$ site and does not leave the ribosome. The EF-G action causes conformational changes of the ribosome from the hyper-rotated to the rotated state. As a result of pseudo translocation, the A-site mRNA SL melts and the P-site tRNA-mRNA anticodon-codon interactions disengage, which allows the forward movement of the ribosome and subsequent sliding towards the landing site. In contrast to canonical translocation, the P-site peptidyl-tRNA ${ }^{\text {Gly }}{ }_{G G A}$ remains attached to the ribosome most probably because of interactions of the nascent peptide with the exit tunnel walls (Fig. 32). Point mutations at the tip of domain IV of EF-G, which lower the rate of canonical translocation, make a higher temperature necessary for efficient bypassing. The mutant factors cannot induce the particular conformation of the decoding center required for the A-site SL melting and rapid P-site tRNA movement, which can be compensated by higher incubation temperatures for efficient bypassing initiation. 
translating ribosome

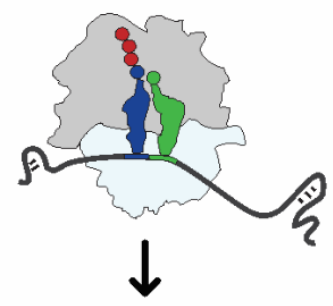

pausing at the take-off site

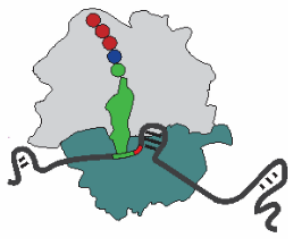

hyper-rotated pseudo translocation
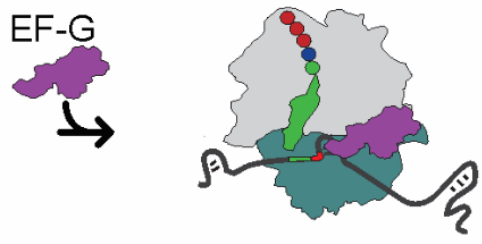

hyper-rotated

to rotated sliding

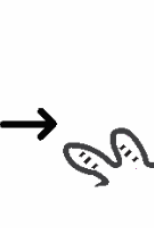

rotated

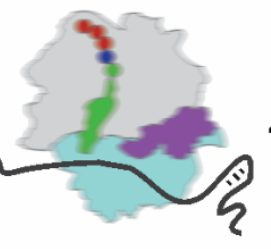

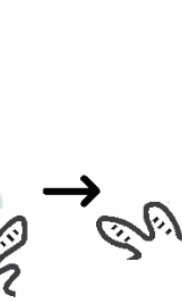

landing

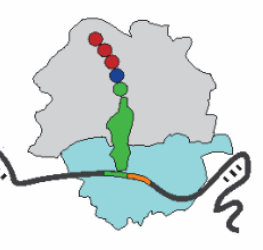

rotated

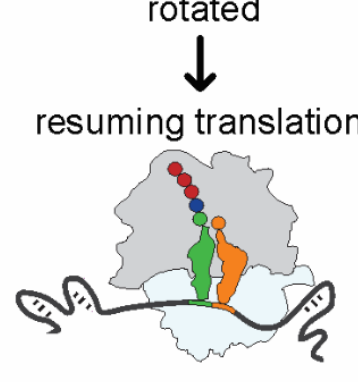

non-rotated

Figure 32. The proposed mechanism of bypassing

Ribosomal conformations are shown with a color gradient from light blue (classical non-rotated state) to green-blue (rotated state) and dark green (hyper-rotated state). The P site peptidyl-tRNA bound to the ribosome during sliding is shown in grass green, the A-site Leu-tRNA ${ }^{\text {Leu } U U A}$ is shown in orange. The blurred cartoon represents the ribosome in motion.

Previous gene 60 bypassing studies suggested that at any time $>97 \%$ of the ribosomes that initiated bypassing successfully move by one nucleotide towards the landing site, whereas only $<3 \%$ of the ribosomes dissociated from the mRNA or lost their P-site peptidyl-tRNA ${ }^{\text {Gly }}{ }_{G G A}$ (Samatova et al., 2014). The substantial distance between the take-off and the landing codon makes an auxiliary factor necessary for directional sliding. We have shown that EF-G makes multiple rounds of GTP hydrolysis as the ribosome slides through the non-coding gap. The GTP consumption during sliding is much higher than during canonical translocation, 1.8 GTP molecules per base compared to approximately one per codon, respectively. The role of EF-G and the reason for the high 
GTP turnover remain to be elucidated. One possibility is that EF-G restricts the backward movement of the ribosome, thereby inducing directionality of sliding or it might be necessary to ensure that the ribosome remains in conformation prone to efficient sliding. Upon EF-G action, the ribosome adopts the rotated conformation, confirming the previous finding that the tRNA corresponding to the $47^{\text {th }}$ codon (first of the ORF2) is accommodated into the rotated ribosome after successful landing (Chen et al., 2015). After landing, translocation resumes with the reading of the $47^{\text {th }}$ codon and A-site tRNA ${ }^{\text {Leu }_{\text {UUA }}}$ accommodation and the ribosome resets into a canonical non-rotated state, which indicates successful bypassing.

Our finding demonstrates that EF-G has additional functions during translation and plays a key role in bypassing on gene 60 mRNA. Moreover, we provide previously unforeseen details of the mechanism of bypassing, which was found to be not only a feature of bacteriophage T4 gene 60 expression but is also inherent to dissimilar organisms (Lang et al., 2014; Nosek et al., 2015). In addition, we provide a glimpse of the mechanism of ribosomal movements through noncoding regions on an mRNA, which may have parallels in other recoding events in prokaryotes. Moreover, recent ribosomal profiling data provided a snapshot of eukaryotic ribosomes which seem to move along noncoding mRNA without recognizing codons; our finding may also serve as a general explanation for the mechanism of how ribosomes move through noncoding parts of mRNAs, such as $3^{\prime}$ untranslated regions of eukaryotic mRNAs (Miettinen \& Bjorklund, 2015; Guydosh \& Green, 2014)

Having the EF-G action as a powerful tool for separating the gene 60 ORF1 and ORF2 translation in time opens the new prospectives for the further bypassing studies. For example, currently available data regarding the pace of sliding and putative scanning prior to the landing requires additional evidence and corroboration (Chen at al., 2015). The identity of the landing codon and 3'SL mRNA structure downstream of the landing codon are necessary for the successful resumption of translation (Herr et al., 2000; Samatova et al., 2014), but the mechanism of landing remains entirely unknown. Further smFRET experiments using various fluorescent reporters introduced not only in the 
ribosome itself but also in other components of the translation system (like mRNA or tRNA) and with EF-G as a bypassing trigger would provide the detailed understanding of the timing of sliding and landing. Moreover, further biochemical studies could bring additional information regarding the motion of the ribosome during gene 60 mRNA translation and help to propose a kinetic model of the bypassing process.

\subsection{The role of ribosomal protein $L 9$ in programmed bypassing in vitro}

We have investigated the role of ribosomal protein L9 in programmed gene 60 translational bypassing in vitro. Although in vivo studies (Herr et al., 2001; Seidman et al., 2011) previously implied a potential regulator activity of the r-protein L9 during translational bypassing, our in vitro data shows that L9 deletion neither increases the bypassing efficiency nor compensates any of the disrupted gene 60 mRNA bypassing signals. We hypothesize therefore that the enhancing effect of L9 deletion previously shown in vivo might be related to the usage of highly mutated mRNAs with a truncated non-coding gap and/or an additional stalling sequence. Deletion of the r-protein L9 leads to a reduced ability of mutated ribosomes to translate long mRNA sequences in vitro, i.e. there is a higher level of spontaneous stalling or drop-off during translation course. We identified that the bypassing efficiency decreases both for intact and mutated ribosomes under conditions of polysome formation in vitro, meaning that fewer ribosomes reach the landing site when they are arranged in polysomes rather than in monosomes. We suggest that coordinated ribosome movements on the structured gene $60 \mathrm{mRNA}$, as well as the optimal ribosome/mRNA ratio, are crucial for successful programmed bypassing. After the "leading" ribosome leaves the take-off codon, the "following" ribosome in the polysome completes the stop product synthesis, but is not able to take-off and bypass, presumably due to the partial unfolding and covering of important mRNA signals. This model is in good agreement with previous gene 60 bypassing studies in vitro indicating the importance of folded 5' and 3'SLs for efficient bypassing (Samatova et al., 2014). Our findings raise questions about the role of L9 in coupling adjacent ribosomes within a polysome during gene $60 \mathrm{mRNA}$ translation in vitro. However, the regulatory activity of L9 within a polysome array would require an extended L9 conformation and only one of several possible orientations adopted by ribosomes within a polysome. Our data might 
also be a result of different packing of single ribosomes on the take-off site and the one recently reported for the colliding ribosomes during -1PRF in vivo (Smith et al., 2019). Those differences may lead to an enlarged distance between the L9 and the mRNA decoding site of the neighboring ribosomes in vitro. As a result, the SSUs of the ribosomes within a polysome array are not juxtaposed and the deletion of L9 does not bring the expected increase in bypassing efficiency in vitro.

Our data provide mechanistic insight into the behavior of colliding ribosomes during gene 60 mRNA translation and allow us to reconsider the influence of r-protein L9 during programmed translational bypassing in vitro. 


\section{Materials and Methods}

\subsection{Materials}

\subsubsection{Equipment}

Table 1. Equipment

\begin{tabular}{|c|c|}
\hline Device & Manufacturer \\
\hline Benchtop centrifuge $5415 R$ & Eppendorf \\
\hline Bio-vision imaging system & Peqlab Biotechnologie \\
\hline Electron multiplier CCD C9100-13 Camera & Hamamatsu \\
\hline Electrophoresis power supply EV261 & Peqlab Biotechnologie \\
\hline FLA-9000 biomolecular imager & Fuji \\
\hline Horizontal gel electrophoresis chamber mini & Peqlab Biotechnologie \\
\hline HPLC System & Waters \\
\hline Incubator shaker series Innova44 & New Brunswick \\
\hline $\begin{array}{c}\text { IX } 81 \text { inverted microscope with PLAPON } 100 \\
\times 1.45 \text { numerical aperture objective }\end{array}$ & Olympus \\
\hline Liquid scintillation counter & PerkinElmer \\
\hline Milli-Q Advantage A10 & Millipore \\
\hline Nanodrop 2000C & Thermo \\
\hline PCR thermocycler Peqstar & Peqlab Biotechnologie \\
\hline $\mathrm{pH}$ meter, $\mathrm{pH}$ electrode & WTW \\
\hline Phosphorimager Fuji Film Fla 7000/9000 & GE Healthcare \\
\hline Plates incubator INE600 & Memmert \\
\hline Spectrophotometer & PerkinElmer \\
\hline SX20 Stopped Flow Spectrometer & Applied Photophysics \\
\hline Ultracentrifuge Optima XPN-100 & Beckmann Coulter \\
\hline $\begin{array}{l}\text { Vertical double gel electrophoresis chamber } \\
\text { maxi }\end{array}$ & Peqlab Biotechnologie \\
\hline $\begin{array}{l}\text { Vertical double gel electrophoresis chamber } \\
\text { mini }\end{array}$ & Peqlab Biotechnologie \\
\hline Vortex Genie 2 & Scientific Industries \\
\hline Water bath RE104 and E100 & Lauda \\
\hline ÄKTA FPLC & GE Healthcare \\
\hline
\end{tabular}


Materials and Methods

\subsubsection{Software}

Table 2. Commercial software packages utilized in the work

\begin{tabular}{|c|c|}
\hline Software & Provider \\
\hline GraphPad Prism & GraphPad software \\
\hline MATLAB & MathWorks \\
\hline MultiGauge & Fujifilm \\
\hline Pymol & Schrödinger \\
\hline TableCurve & Systat Software GmbH \\
\hline
\end{tabular}

\subsubsection{Chemicals and consumables}

Chemicals were purchased from Sigma Aldrich, Roche Molecular Biochemicals, Serva or Merck, unless stated otherwise. GTP from Jena Bioscience, dNTP from New England BioLabs, fluorescent dyes from GE Healthcare Life Sciences, BSA and NeutrAvidin from ThermoFisher, protocatechuate-3,4-dioxygenase from Pseudomonas, kits for DNA preparation from Macherey-Nagel. Radioactive compounds were from Hartmann Analytic. Scintillation liquid Ultima Gold ${ }^{\mathrm{TM}} \mathrm{XR}$ and Quickszint 361 were from PerkinEImer and Zinsser Analytics, respectively. Nitrocellulose filters, sterile filters were from Sartorius, centrifuge tubes from Beckman Coulter, cellulose plates CEL 300 TLC from Macherey-Nagel. Biotin-polyethylene glycol quartz slides prepared as described (Adio et al., 2015).

\subsubsection{Enzymes}

Table 3. List of enzymes used

\begin{tabular}{|c|c|}
\hline Enzyme & Manufacturer \\
\hline Dpnl & NEB \\
\hline Phusion® High-Fidelity DNA Polymerase & Thermo Fisher \\
\hline Pyrophosphatase & Sigma-Aldrich \\
\hline Pyruvate Kinase & Roche \\
\hline T7 RNA Polymerase & $\begin{array}{c}\text { Provided by the laboratory } \\
\text { facility }\end{array}$ \\
\hline
\end{tabular}




\subsubsection{Buffers}

Table 4. Common buffers

\begin{tabular}{|c|c|}
\hline Buffer & Components \\
\hline LB agar & $\begin{array}{l}10 \mathrm{~g} / \mathrm{l} \mathrm{NaCl} \\
10 \mathrm{~g} / \mathrm{l} \mathrm{tryptone} \\
5 \mathrm{~g} / \mathrm{l} \text { yeast extract } \\
15 \mathrm{~g} / \mathrm{l} \text { agar }\end{array}$ \\
\hline LB medium & $\begin{array}{l}10 \mathrm{~g} / \mathrm{l} \mathrm{NaCl} \\
10 \mathrm{~g} / \mathrm{l} \text { tryptone } \\
5 \mathrm{~g} / \mathrm{l} \text { yeast extract }\end{array}$ \\
\hline 10X TAE & $\begin{array}{l}0.4 \mathrm{M} \text { Tris- } \mathrm{HCl}, \mathrm{pH} 8.4 \\
0.2 \mathrm{M} \text { acetic acid } \\
10 \mathrm{mM} \text { EDTA }\end{array}$ \\
\hline 10X TBE & $\begin{array}{l}0.89 \mathrm{M} \text { Tris- } \mathrm{HCl}, \mathrm{pH} 8.3 \\
0.89 \mathrm{M} \text { boric acid } \\
25 \mathrm{mM} \text { EDTA }\end{array}$ \\
\hline 2X RNA loading dye & $\begin{array}{l}2 \mathrm{mM} \text { Tris-HCl, pH } 7.5 \\
20 \mathrm{mM} \text { EDTA } \\
8 \mathrm{M} \text { urea } \\
20 \% \text { glycerol } \\
0.05 \% \text { (w/v) bromophenol } \\
\text { blue } \\
0.05 \%(\mathrm{w} / \mathrm{v}) \text { xylene cyanol }\end{array}$ \\
\hline 5X mRNA transcription buffer & 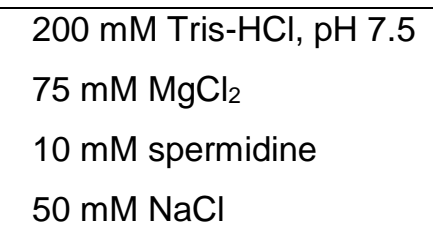 \\
\hline \multicolumn{2}{|c|}{ In vitro translation buffers } \\
\hline 1X TAKM 7 buffer & 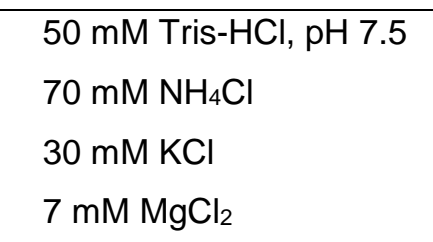 \\
\hline 1X TAKM 50 buffer & 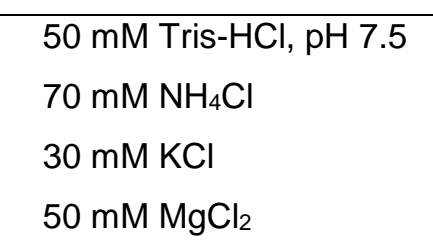 \\
\hline
\end{tabular}




\begin{tabular}{|c|c|}
\hline 1X HiFi buffer & 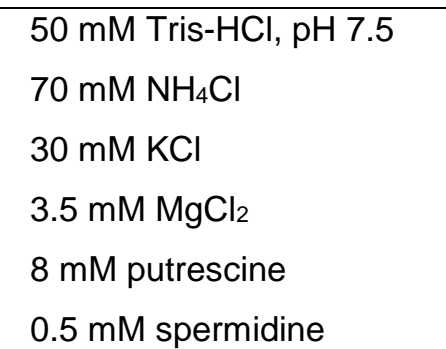 \\
\hline \multicolumn{2}{|c|}{ smFRET buffers } \\
\hline S6 reconstitution buffer & $\begin{array}{l}50 \mathrm{mM} \text { HEPES-KOH, pH7.5 } \\
400 \mathrm{mM} \mathrm{KCl} \\
4 \mathrm{mM} \mathrm{MgCl} 2 \\
6 \mathrm{mM} \beta-\text { mercaptoethanol } \\
5 \%(w / w) \text { glycerol }\end{array}$ \\
\hline L9 reconstitution buffer & $\begin{array}{l}50 \mathrm{mM} \text { HEPES-KOH, pH7.5 } \\
400 \mathrm{mM} \mathrm{NH}_{4} \mathrm{Cl} \\
4 \mathrm{mM} \mathrm{MgCl}_{2} \\
6 \mathrm{mM} 2-\mathrm{mercaptoethanol} \\
5 \%(\mathrm{w} / \mathrm{w}) \text { glycerol }\end{array}$ \\
\hline Blocking buffer & $\begin{array}{l}50 \mathrm{mM} \text { Tris- } \mathrm{HCl}, \mathrm{pH} 7.5 \\
70 \mathrm{mM} \mathrm{NH}_{4} \mathrm{Cl} \\
30 \mathrm{mM} \mathrm{KCl} \\
3.5 \mathrm{mM} \mathrm{MgCl}_{2} \\
8 \mathrm{mM} \text { putrescine } \\
0.5 \mathrm{mM} \text { spermidine } \\
10 \mathrm{mg} / \mathrm{ml} \mathrm{BSA} \\
1 \mu \mathrm{M} \text { NeutrAvidin }\end{array}$ \\
\hline Washing buffer & $\begin{array}{l}50 \mathrm{mM} \text { Tris- } \mathrm{HCl}, \mathrm{pH} 7.5 \\
70 \mathrm{mM} \mathrm{NH} 4 \mathrm{Cl} \\
30 \mathrm{mM} \mathrm{KCl} \\
3.5 \mathrm{mM} \mathrm{MgCl} 2 \\
8 \mathrm{mM} \text { putrescine } \\
0.5 \mathrm{mM} \text { spermidine } \\
1 \mathrm{mg} / \mathrm{ml} \mathrm{BSA}\end{array}$ \\
\hline Imaging buffer & $\begin{array}{l}50 \mathrm{mM} \text { Tris- } \mathrm{HCl}, \mathrm{pH} 7.5 \\
70 \mathrm{mM} \mathrm{NH}_{4} \mathrm{Cl} \\
30 \mathrm{mM} \mathrm{KCl} \\
3.5 \mathrm{mM} \mathrm{MgCl}_{2} \\
8 \mathrm{mM} \text { putrescine }\end{array}$ \\
\hline
\end{tabular}




\begin{tabular}{|c|c|}
\hline & $\begin{array}{l}0.5 \mathrm{mM} \text { spermidine } \\
5 \mathrm{mM} \text { protocatechuic acid } \\
50 \mathrm{nM} \text { protocatechuate-3,4- } \\
\text { dioxygenase }\end{array}$ \\
\hline \multicolumn{2}{|c|}{ Von Jagow Tris-Tricine SDS PAGE buffers } \\
\hline 10X Cathode buffer & $\begin{array}{l}1 \mathrm{M} \text { Tris- } \mathrm{HCl}, \mathrm{pH} 8.25 \\
1 \mathrm{M} \text { Tricine } \\
1 \%(\mathrm{w} / \mathrm{w}) \mathrm{SDS}\end{array}$ \\
\hline 10X Anode buffer & 2 M Tris-HCl pH 8.9 \\
\hline 2X Sample buffer & $\begin{array}{l}0.1 \mathrm{M} \text { Tris-HCl, } \mathrm{pH} 6.8 \\
0.28 \mathrm{M} \beta \text {-mercaptoethanol } \\
24 \% \text { (w/v) glycerol } \\
10 \% \text { SDS }\end{array}$ \\
\hline $3 X$ Gel buffer & $\begin{array}{l}3 \mathrm{M} \text { Tris-HCl, pH } 8.45 \\
0.3 \% \text { SDS }\end{array}$ \\
\hline $49.5 \% \mathrm{~T}, 3 \% \mathrm{C}$ mixture of acrylamide/bis & $\begin{array}{l}480 \mathrm{~g} / \mathrm{l} \text { acrylamide } \\
15 \mathrm{~g} / \mathrm{l} \text { bis-acrylamide }\end{array}$ \\
\hline $49.5 \% \mathrm{~T}, 6 \% \mathrm{C}$ mixture of acrylamide/bis & $\begin{array}{l}465 \mathrm{~g} / \mathrm{l} \text { acrylamide } \\
30 \mathrm{~g} / \mathrm{l} \text { bis-acrylamide }\end{array}$ \\
\hline \multicolumn{2}{|c|}{ Peptide separation buffers, HPLC } \\
\hline Buffer A & $0.1 \%$ trifluoroacetic acid \\
\hline Buffer B & $\begin{array}{l}0.1 \% \text { trifluoroacetic acid } \\
65 \% \text { acetonitrile }\end{array}$ \\
\hline \multicolumn{2}{|c|}{ mRNA purification buffers, FPLC } \\
\hline Buffer A & $\begin{array}{l}30 \mathrm{mM} \text { Bis-Tris, pH } 6.0 \\
1 \mathrm{mM} \text { EDTA } \\
300 \mathrm{mM} \mathrm{NaCl}\end{array}$ \\
\hline Buffer B & $\begin{array}{l}30 \mathrm{mM} \text { Bis-Tris, pH } 6.0 \\
1 \mathrm{mM} \text { EDTA } \\
1.5 \mathrm{M} \mathrm{NaCl}\end{array}$ \\
\hline \multicolumn{2}{|c|}{ Total tRNA purification buffers, FPLC } \\
\hline Buffer A & $\begin{array}{l}50 \mathrm{mM} \mathrm{NaOAc}, \mathrm{pH} 4.5 \\
10 \mathrm{mM} \mathrm{MgCl}_{2}\end{array}$ \\
\hline Buffer B & $\begin{array}{l}50 \mathrm{mM} \mathrm{NaOAc}, \mathrm{pH} 4.5 \\
10 \mathrm{mM} \mathrm{MgCl}_{2}\end{array}$ \\
\hline
\end{tabular}


Materials and Methods

\section{$1.1 \mathrm{M} \mathrm{KCl}$}

\subsubsection{E coli strains}

Table 5. Bacterial strains

\begin{tabular}{|l|l|}
\hline NovaBlue & Merck \\
\hline BW25113 & DMSZ \\
\hline MDS57 & Prof. Harry Noller \\
\hline
\end{tabular}

\subsubsection{Plasmids}

\begin{tabular}{|l|l|}
\hline pT60.32 & Prof. John Atkins \\
\hline
\end{tabular}

\subsubsection{Columns}

Table 6. Purification columns

\begin{tabular}{|l|l|}
\hline Column & Manufacturer \\
\hline BioSuite 450 HR 8-mm & Waters \\
\hline LiChrospher 100 RP-8 & Merck \\
\hline HiTrap Q HP 5ml & GE Healthcare \\
\hline
\end{tabular}

\subsubsection{DNA oligonucleitodes}

Table 7. Primers for gene 60 constructs (IBA Lifesciences or Eurofins Genomics)

\begin{tabular}{|c|c|c|}
\hline Name & Sequence $\mathbf{5}^{\prime} \rightarrow \mathbf{3}^{\prime}$ & Function \\
\hline & mRNA transcription templates and consctructs sequencing & \\
\hline MR4 direct & TCACCGTCATCACCGAAA & $\begin{array}{c}\text { Fwd } \\
\text { primer for } \\
\text { transcripti } \\
\text { on } \\
\text { templates } \\
\text { and } \\
\text { sequencin } \\
\text { g }\end{array}$ \\
\hline MR4 reverse & CTACTGGCTCATCCATTCTTTACG & $\begin{array}{c}\text { Rev } \\
\text { primer for } \\
\text { transcripti } \\
\text { on } \\
\text { templates }\end{array}$ \\
\hline
\end{tabular}




\begin{tabular}{|c|c|c|}
\hline STOP reverse & CCATCGTGATCTGCGTCTG & $\begin{array}{c}\text { Rvs primer } \\
\text { for } \\
\text { transcripti } \\
\text { on } \\
\text { templates } \\
\text { truncated } \\
\text { after } \\
\text { ORF1 }\end{array}$ \\
\hline \multicolumn{3}{|c|}{ Mutations in main SL 5'GGATAGCCTTCGGGCTATCTATAG3' } \\
\hline minusGC_dir & $\begin{array}{l}\text { GACAGACGCAGATCACGATGGATAGCTTCGGCTATCTATAGAAATA } \\
\text { CCTC }\end{array}$ & \multirow{14}{*}{$\begin{array}{l}\text { Mutation } \\
\text { in main SL }\end{array}$} \\
\hline minusGC_rev & $\begin{array}{l}\text { GAGGTATTTCTATAGATAGCCGAAGCTATCCATCGTGATCTGCGTC } \\
\text { TGTC }\end{array}$ & \\
\hline plusGC_dir & CGCAGATCACGATGGATAGCCCTTCGGGGCTATCTATAGAAATACC & \\
\hline plusGC_rev & GGTATTTCTATAGATAGCCCCGAAGGGCTATCCATCGTGATCTGCG & \\
\hline BX1z_dir & CGCAGATCACGATGGATAGCGTTCGGCCTATCTATAGAAATACC & \\
\hline BX1z_rev & GGTATTTCTATAGATAGGCCGAACGCTATCCATCGTGATCTGCG & \\
\hline BX5b_dir & $\begin{array}{l}\text { GACGCAGATCACGATGGATAGCCTCGACTGAGGCTATCTATAGAAA } \\
\text { TACCTC }\end{array}$ & \\
\hline BX5b_rev & $\begin{array}{l}\text { GAGGTATTTCTATAGATAGCCTCAGTCGAGGCTATCCATCGTGATC } \\
\text { TGCGTC }\end{array}$ & \\
\hline BX1d_dir & CGCAGATCACGATGGATAGCGTTCGGGCTATCTATAG & \\
\hline BX1d_rev & CTATAGATAGCCCGAACGCTATCCATCGTGATCTGCG & \\
\hline BX5c_dir & $\begin{array}{l}\text { GACAGACGCAGATCACGATGGATAGCCTCGACTCGAGTCGAGGCT } \\
\text { ATCTATAGAAATACCTC }\end{array}$ & \\
\hline BX5c_rev & $\begin{array}{l}\text { GAGGTATTTCTATAGATAGCCTCGACTCGAGTCGAGGCTATCCATC } \\
\text { GTGATCTGCGTCTGTC }\end{array}$ & \\
\hline BX1x_dir & $\begin{array}{l}\text { GACGCAGATCACGATGGATAGGGTTCGCCCTATCTATAGAAATACC } \\
\text { TC }\end{array}$ & \\
\hline BX1x_rev & $\begin{array}{l}\text { GAGGTATTTCTATAGATAGGGCGAACCCTATCCATCGTGATCTGCG } \\
\text { TC }\end{array}$ & \\
\hline \multicolumn{3}{|c|}{ Mutations in 5' SL 5'TGCGAATGTCGCTATTATGACAGACGCA3' } \\
\hline 4 mut_dir & CGCAAACGTTGCTATTATGACAGACGCAGATCACGATGGATA & \multirow{2}{*}{$\begin{array}{l}\text { Mutation } \\
\text { in } 5 \text { ' SL }\end{array}$} \\
\hline 4 mut_rev & TATCCATCGTGATCTGCGTCTGTCATAATAGCAACGTTTGCG & \\
\hline $\begin{array}{l}4 \\
\text { mut_comp_dir }\end{array}$ & CGCAAACGTTGCTATTATAACGGATGCGGATCACGATGGATA & \multirow{2}{*}{$\begin{array}{l}\text { Compens } \\
\text { atory } \\
\text { mutation } \\
\text { in } 5^{\prime} \mathrm{SL}\end{array}$} \\
\hline mut_comp_re & TATCCATCGTGATCCGCATCCGTTATAATAGCAACGTTTGCG & \\
\hline \multicolumn{3}{|c|}{ Mutations in nascent peptide segment KKYKLQNNVRRSIKSSS ${ }^{14-30}$} \\
\hline 1K_NP_DIR & CTTCTAGCGTTGATATGGAAAAATATAAATTGCAG & \multirow{2}{*}{$\mathrm{K} 14 \rightarrow \mathrm{E}$} \\
\hline 1K_NP_REV & CTGCAATTTATATTTTTCCATATCAACGCTAGAAG & \\
\hline 2K_NP_DIR & CTAGCGTTGATATGAAAGAATATAAATTGCAGAAC & \multirow{2}{*}{$\mathrm{K} 15 \rightarrow \mathrm{E}$} \\
\hline 2K_NP_REV & GTTCTGCAATTTATATTCTTTCATATCAACGCTAG & \\
\hline 3Y_NP_DIR & GCGTTGATATGAAAAAACATAAATTGCAGAACAATG & \multirow{2}{*}{$\mathrm{Y} 16 \rightarrow \mathrm{H}$} \\
\hline 3Y_NP_REV & CATTGTTCTGCAATTTATGTTTTTTCATATCAACGC & \\
\hline
\end{tabular}


Materials and Methods

\begin{tabular}{|c|c|c|}
\hline 4K_NP_DIR & GATATGAAAAAATATGAATTGCAGAACAATGTTCG & \multirow{2}{*}{$\mathrm{K} 17 \rightarrow \mathrm{E}$} \\
\hline 4K_NP_REV & CGAACATTGTTCTGCAATTCATATTTTTTCATATC & \\
\hline 5L_NP_DIR & GATATGAAAAAATATAAATCGCAGAACAATGTTCGTCG & \multirow{2}{*}{$\mathrm{L} 18 \rightarrow \mathrm{S}$} \\
\hline 5L_NP_REV & CGACGAACATTGTTCTGCGATTTATATTTTTTCATATC & \\
\hline 6Q_NP_DIR & GATATGAAAAAATATAAATTGGAGAACAATGTTCGTCG & \multirow{2}{*}{$\mathrm{Q} 19 \rightarrow \mathrm{E}$} \\
\hline 6Q_NP_REV & CGACGAACATTGTTCTCCAАTTTATATTTTTTCATATC & \\
\hline 7N_NP_DIR & GAAAAAATATAAATTGCAGAAGAATGTTCGTCGTTC & \multirow{2}{*}{$\mathrm{N} 20 \rightarrow \mathrm{K}$} \\
\hline 7N_NP_REV & GAACGACGAACATTCTTCTGCAATTTATATTTTTTC & \\
\hline 8N_NP_DIR & GAAAAAATATAAATTGCAGAACAAGGTTCGTCGTTC & \multirow{2}{*}{$\mathrm{N} 21 \rightarrow \mathrm{K}$} \\
\hline 8N_NP_REV & GAACGACGAACCTTGTTCTGCAATTTATATTTTTTC & \\
\hline 9V_NP_DIR & GCAGAACAATGATCGTCGTTCTATTAAATCCTCTTC & \multirow{2}{*}{$\mathrm{V} 22 \rightarrow \mathrm{D}$} \\
\hline 9V_NP_REV & GAAGAGGATTTAATAGAACGACGATCATTGTTCTGC & \\
\hline 10R_NP_DIR & GCAGAACAATGTTCTTCGTTCTATTAAATCCTCTTC & \multirow{2}{*}{$\mathrm{R} 23 \rightarrow \mathrm{L}$} \\
\hline 10R_NP_REV & GAAGAGGATTTAATAGAACGAAGAACATTGTTCTGC & \\
\hline 11R_NP_DIR & GCAGAACAATGTTCGTCTTTCTATTAAATCCTCTTC & \multirow{2}{*}{$\mathrm{R} 24 \rightarrow \mathrm{L}$} \\
\hline 11R_NP_REV & GAAGAGGATTTAATAGAAAGACGAACATTGTTCTGC & \\
\hline 12S_NP_DIR & GAACAATGTTCGTCGTTTTATTAAATCCTCTTCAATG & \multirow{2}{*}{$\mathrm{S} 25 \rightarrow \mathrm{F}$} \\
\hline 12S_NP_REV & CATTGAAGAGGATTTAATAAAACGACGAACATTGTTC & \\
\hline 13I_NP_DIR & CAATGTTCGTCGTTCTTTTAAATCCTCTTCAATGAAC & \multirow{2}{*}{$\mathrm{I} 26 \rightarrow \mathrm{F}$} \\
\hline 13I_NP_REV & GTTCATTGAAGAGGATTTAAAAGAACGACGAACATTG & \\
\hline 14K_NP_DIR & CGTCGTTCTATTATATCCTCTTCAATGAACTATGCG & \multirow{2}{*}{$\mathrm{K} 27 \rightarrow \mathrm{I}$} \\
\hline 14K_NP_REV & CGCATAGTTCATTGAAGAGGATATAATAGAACGACG & \\
\hline 15S_NP_DIR & CGTCGTTCTATTAAATTCTCTTCAATGAACTATGCG & \multirow{2}{*}{$\mathrm{S} 28 \rightarrow \mathrm{F}$} \\
\hline 15S_NP_REV & CGCATAGTTCATTGAAGAGAATTTAATAGAACGACG & \\
\hline 16S_NP_DIR & CGTCGTTCTATTAAATCCTTTTCAATGAACTATGCG & \multirow{2}{*}{$\mathrm{S} 29 \rightarrow \mathrm{F}$} \\
\hline 16S_NP_REV & CGCATAGTTCATTGAAAAGGATTTAATAGAACGACG & \\
\hline 17S_NP_DIR & CGTTCTATTAAATCCTCTTTAATGAACTATGCGAATG & \multirow{2}{*}{$\mathrm{S} 30 \rightarrow \mathrm{L}$} \\
\hline 17S_NP_REV & CATTCGCATAGTTCATTAAAGAGGATTTAATAGAACG & \\
\hline $\begin{array}{l}\text { KKYK_NP_DI } \\
\text { R }\end{array}$ & $\begin{array}{l}\text { GATTCTTCTAGCGTTGATATGGAAGAACATGAATTGCAGAACAATGT } \\
\text { TCGTCGTTC }\end{array}$ & \multirow{2}{*}{$\begin{array}{l}\mathrm{KKYK} \rightarrow \\
\mathrm{EEHE}\end{array}$} \\
\hline $\begin{array}{l}\text { KKYK_NP_RE } \\
\text { V }\end{array}$ & $\begin{array}{l}\text { GAACGACGAACATTGTTCTGCAATTCATGTTCTTCCATATCAACGCT } \\
\text { AGAAGAATC }\end{array}$ & \\
\hline \multicolumn{3}{|c|}{ smFRET annealing biotinylated oligonucleotide } \\
\hline $\begin{array}{l}\text { DNA- } \\
\text { biotin_bypassi } \\
\text { ng }\end{array}$ & CCGCGTTTGGGCGAGCTCGAATTCCGGTCTCCC - biotin & $\begin{array}{l}\text { Immobiliza } \\
\text { tion of } \\
\text { mRNA on } \\
\text { a slide }\end{array}$ \\
\hline
\end{tabular}




\subsubsection{0. mRNA}

Table 8. Sequences of wt mRNAs

\begin{tabular}{|c|c|}
\hline mRNA & Sequence $5^{\prime} \rightarrow 3^{\prime}$ \\
\hline Gene 60 WT mRNA* & $\begin{array}{l}\text { GGGAGACCGGAAUUCGAGCUCGCCCAAACG } \\
\text { CGGUUGGAUUCCUGAUGAAAGUUCUAUGA } \\
\text { GGUGUAUAAUGAAAUUUGUAAAUUGAUUC } \\
\text { UUCUAGCGUUGAUAUGAAAAAUAUAAAUUG } \\
\text { CAGAACAAUGUUCGUCGUUCUAUUAAUCCU } \\
\text { CUUCAAUGAACUAUGCGAAUGUCGCUAUUAU } \\
\text { GACAGACGCAGAUCACGAUGGAUAGCCUUC } \\
\text { GGGCUAUCUAUAGAAAUACCUCAUAAUUAAG } \\
\text { AGAUUAUUGGAUUAGGUUCUAUUUAUCCUUC } \\
\text { UCUGCUCGGAUUUUUUAGUAAUGGCCAGA } \\
\text { AUUGUUUGAGCAAGGACGAAUUCGCUUUGU } \\
\text { CAAAACUCCUGUAAUCAUCGCUCAGGUCGGU } \\
\text { AAAAAACAAGAAUGGUUUUAUACAGUCGCUG } \\
\text { AAUAUGAGAGUGCCAAAGAUGCUCUACCUAA } \\
\text { ACAUAGCAUCCGUUAUAUUAAGGGACUUGGC } \\
\text { UCUUUGGAAAAUCUGAAUAUCGUGAGAUGA } \\
\text { UUCAAAACCCAGUAUAUGAUGUUGUUAAACU } \\
\text { UCCUGAGAACUGGAAAGAGCUUUUUGAAAUG } \\
\text { CUCAUGGGAGAUAAUGCUGACCUUCGUAAA } \\
\text { GAAUGGAUGAGCCAGUAG }\end{array}$ \\
\hline $\begin{array}{l}\text { Gene } 60 \text { mRNA truncated after the first stop } \\
\text { codon }(\mathrm{ORF} 1)^{\star}\end{array}$ & $\begin{array}{l}\text { GGGAGACCGGAAUUCGAGCUCGCCCAAACG } \\
\text { CGGUUGGAUUCCUGAUGAAAAUUCUAUGA } \\
\text { GGUGUAUAAUGAAAUUUGUAAAAAUUGUUC } \\
\text { UUCUAGCGUUGAUAUGAAAAAUAUAAAUUG } \\
\text { CAGAACAAUGUUCGUCGUUCUAUUAAAUCCU } \\
\text { CUUCAAUGAACUAUGCGAAUGUCGCUAUUAU } \\
\text { GACAGACGCAGAUCACGAUGGAUAG }\end{array}$ \\
\hline
\end{tabular}


Materials and Methods

\begin{tabular}{|l|l|}
\hline SecM mRNA (provided by Marija Liutkute) & AUUAAUACGACUCACUAUAGGGGAAUUGUGA \\
& GCGGAUAACAAUUCCCCUCUAGAAAUAAUUU \\
& UGUUUACUUUAAGAGGAGAUAUACAUAUG \\
& GAAUAUCAACACUGGUUACGUGAAGCAAUAA \\
& GCCAACUUCAGGCGAGCGAAAGCCGCGGC \\
& GUGAUGCUGAAAUCCUGCUGGAACAUGUUA \\
& CCGGCAAAGGGCGUACUUUUAUCCUCGCCU \\
& UUGGUGAAACGCAGCUGACUGACGAACAAU \\
& GUCAGCAACUUGAUGCGCUACUGACACGUC \\
& GUCGCGAUGGUGAACCCAUUGCUCAUUUAA \\
& CCGGGGUGCGAGAUUUCUGGUCGUUGCCGU \\
& UAUUCAGCACGCCCGUCUGGAUAAGCCAGG \\
& CGCAAGGCAUCCGUGCUGGCCCUAUGUCCG \\
& GUAAAAUGACUGGUAUCGUAAAAUGGUUCAA \\
& CGCUGACAAAGGCUUCGGCUUCAUCACUCC \\
& U \\
\hline mMF mRNA (IBA Lifesciences) & GUUACAGGUAUACAUACUAUGGUGUUCAUU \\
\hline mMF+14Alx405 (IBA Lifesciences) & AC \\
\hline & GUUAACAGGUAUACAUACUAUGGUGUUCAUU \\
& AC-AIx405 \\
\hline
\end{tabular}

${ }^{*}$ AUG start codon is highlighted in yellow, UAG stop codon in red, take-off and landing GGA codons in green, NP segment KKYKLQNNVRRSIKSSS encoding sequence in turquoise, A-site SL in bold, the region for biotinylated primer annealing (smFRET experiments) is in grey;

${ }^{* *}$ AUG start codon is highlighted in yellow, SecM FSTPVWISQAQGIRAGP encoding sequence is in pink.

\subsection{Methods}

\subsubsection{Template pDNA construction by polymerase chain reaction (PCR)}

Insertions, deletions and nucleotide substitutions were introduced in pDNA by PCR (Saiki et al., 1988) according to Table 9-10. Forward and reverse primers for mutations are summarized in Table 7. 
Table 9. PCR mix for mutagenesis

\begin{tabular}{|c|c|}
\hline Component & $\begin{array}{c}\text { Final } \\
\text { concentration }\end{array}$ \\
\hline $\begin{array}{c}\text { Phusion reaction } \\
\text { buffer }\end{array}$ & $1 \mathrm{X}$ \\
\hline Template pDNA & $0.04 \mu \mathrm{g} / \mu \mathrm{l}$ \\
\hline Forward primer & $0.4 \mu \mathrm{M}$ \\
\hline Reverse primer & $0.4 \mu \mathrm{M}$ \\
\hline dNTP mix & $0.4 \mathrm{mM}$ \\
\hline Phusion polymerase & $0.12 \mathrm{u} / \mu \mathrm{l}$ \\
\hline
\end{tabular}

Table 10. PCR program for mutagenesis

\begin{tabular}{|c|c|c|c|}
\hline Cycle step & Temperature, $^{\circ} \mathbf{C}$ & Time, s & Number of cycles \\
\hline Initial denaturation & 98 & 30 & 1 \\
\cline { 1 - 3 } Denaturation & 98 & 10 & \multirow{2}{*}{25} \\
\hline Primer annealing & 55 & 30 & \\
\hline Extension & 72 & 90 & 1 \\
\hline Final extension & 72 & 600 & \\
\hline
\end{tabular}

After the PCR the reaction mixture was incubated with Dpnl $(0.04 \mathrm{u} / \mu \mathrm{l})$ for 1 hour at $37^{\circ} \mathrm{C}$ to digest the template pDNA, which is methylated as opposed to newly synthesized DNA.

\subsubsection{Transformation and purification of pDNA}

PCR products (10-200 ng) were transformed into NovaBlue E. coli competent cells $(50 \mu \mathrm{l})$. Competent cells were incubated with PCR product on ice for $30 \mathrm{~min}$ and then heat-shocked at $42^{\circ} \mathrm{C}$ for $45 \mathrm{~s}$. After heat shock transformation cells were kept on ice for $3 \mathrm{~min}$ for recovery and then incubated in LB medium for $45 \mathrm{~min}$ at $37^{\circ} \mathrm{C}$ in a waterbath. The cells were harvested by centrifugation for $5 \mathrm{~min}$ at $3000 \mathrm{rpm}$ at RT, plated on LB agar containing ampicillin $(100 \mu \mathrm{g} / \mathrm{ml})$ and grown overnight at $37^{\circ} \mathrm{C}$. A single colony was inoculated in $5 \mathrm{ml}$ of LB medium supplemented with ampicillin $(100 \mu \mathrm{g} / \mathrm{ml})$ and grown overnight at $37^{\circ} \mathrm{C}$ constant shaken (200 rpm). The cells were harvested by centrifugation 
for $30 \mathrm{~s}$ at $11000 \mathrm{rpm}$ at $22^{\circ} \mathrm{C}$, and pDNA was purified using the Macherey-Nagel Plasmid Preparation Kit (Mini scale) according to the manufacturer's protocols. Quality and purity of the pDNA were checked by spectrophotometric measurements at $260 \mathrm{~nm}$ and agarose gel electrophoresis (AGE). Samples for AGE were prepared by mixing the DNA (100-200 ng) with 6X Loading dye (Purple loading dye, New England Biolabs). Agarose gels (1\%) were pre-stained with Stain $\mathrm{G}$ (1:100 000 dilution). AGE was performed in 1XTAE running buffer at $200 \mathrm{~V}$ for $30 \mathrm{~min}$ and bands were detected in a UV transilluminator.

\subsection{3. mRNA preparation}

Short DNA linear segments were used as in vitro transcription templates and synthesized by PCR (Saiki et al., 1988) according to Table 11-12. Forward and reverse primers for amplification are summarized in Table 7.

Table 11. PCR mix for mRNA preparation

\begin{tabular}{|c|c|}
\hline Component & $\begin{array}{c}\text { Final } \\
\text { concentration }\end{array}$ \\
\hline $\begin{array}{c}\text { Phusion reaction } \\
\text { buffer }\end{array}$ & $1 \mathrm{X}$ \\
\hline Template pDNA & $0.1 \mathrm{ng} / \mu \mathrm{l}$ \\
\hline Forward primer & $0.4 \mu \mathrm{M}$ \\
\hline Reverse primer & $0.4 \mu \mathrm{M}$ \\
\hline dNTP mix & $0.2 \mathrm{mM}$ \\
\hline Phusion polymerase & $2 \mathrm{u} / \mu \mathrm{l}$ \\
\hline
\end{tabular}

Table 12. PCR program for mRNA preparation

\begin{tabular}{|c|c|c|c|}
\hline Cycle step & Temperature, $^{\circ} \mathbf{C}$ & Time, $\mathbf{s}$ & Number of cycles \\
\hline Initial denaturation & 98 & 30 & 1 \\
\hline Denaturation & 98 & 10 & \multirow{2}{*}{35} \\
\hline Primer annealing & 55 & 20 & \\
\hline Extension & 72 & 30 & \\
\hline
\end{tabular}


In vitro transcription was carried out using T7 RNA polymerase (Gurevich et al., 1991) according to Table 13 for $4 \mathrm{~h}$ at $37^{\circ} \mathrm{C}$.

Table 13. In vitro transcription mix

\begin{tabular}{|c|c|}
\hline Component & $\begin{array}{c}\text { Final } \\
\text { concentration }\end{array}$ \\
\hline TAB buffer & $1 \mathrm{X}$ \\
\hline DTT & $10 \mathrm{mM}$ \\
\hline NTP & $3 \mathrm{mM}$ \\
\hline GMP & $5 \mathrm{mM}$ \\
\hline PCR DNA template & $100 \mathrm{ng} / \mu \mathrm{l}$ \\
\hline Pyrophosphatase & $0.01 \mathrm{u} / \mu \mathrm{l}$ \\
\hline RNAse inhibitor & $0.2 \mathrm{U} / \mu \mathrm{l}$ \\
\hline T7 RNA polymerase & $1.6 \mathrm{u} / \mu \mathrm{l}$ \\
\hline
\end{tabular}

Products of preparative in vitro transcription were purified on a HiTrap Q HP anionexchange column using fast protein liquid chromatography (FPLC); fractions were analyzed by spectrophotometric measurements at $260 \mathrm{~nm}$. Peak fractions were pooled according to the elution profile and precipitated overnight with $1 / 10 \mathrm{~V} \mathrm{KOAc}(20 \%, \mathrm{pH}$ 5.0) and $2.5 \mathrm{~V}$ ethanol at $-20^{\circ} \mathrm{C}$. The mRNA was pelleted by centrifugation for $1 \mathrm{~h}$ at 4000 rpm at $4^{\circ} \mathrm{C}$ and dissolved in Milli-Q H2O. Quality and purity of mRNAs were checked by spectrophotometric measurements at $260 \mathrm{~nm}$ and 10\% urea polyacrylamide gel electrophoresis (urea-PAGE) performed in $1 \mathrm{X}$ TBE buffer at $150 \mathrm{~V}$ for $3 \mathrm{~h}$ at RT. Gels were fixed by $3 \mathrm{~min}$ incubaton in $10 \% \mathrm{CH} 3 \mathrm{COOH}$ and stained with $0.04 \%$ methylene blue in $2 \mathrm{M} \mathrm{CH} 3 \mathrm{COONa}$ solution ( $\mathrm{pH}$ 5.0) for $1 \mathrm{~h}$. Gels were destained with $\mathrm{H} 2 \mathrm{O}$ overnight. To estimate the yield, the molar mRNA concentrations were calculated by the following formula:

$$
\mathrm{C}_{\mathrm{mRNA}}, \mathrm{mol} / \mathrm{L}=\frac{\left[\mathrm{C}_{\mathrm{mRNA}}\right], \mathrm{g} / \mathrm{L}}{[(\# \text { nucleotides } \times 320.5)+159.0], \mathrm{g} / \mathrm{mol}}
$$

where $320.5 \mathrm{~g} / \mathrm{mol}$ is the average molecular weight of ribonucleotide monophosphates; addition of $159.0 \mathrm{~g} / \mathrm{mol}$ takes into account the 5 ' triphosphate on ssRNA. 
Materials and Methods

\subsubsection{TRNA preparation}

fMet-tRNA $^{\text {fMet }}$, BODIPY-Met-tRNA ${ }^{\text {fMet }}$ were prepared as described (Rodnina et al., 1994; Milon et al., 2007) and provided by the laboratory facility. $\left[{ }^{14} \mathrm{C}\right]$ Leu-tRNA ${ }^{\text {Leu }}$ UUA was prepared as described (Korniy et al., 2019) and provided by Natalia Korniy.

Total aminoacylated tRNA was prepared from E. coli total tRNA (Roche) and aminoacylated with a mixture of synthetases (contained in S100 E. coli cell extract, inhouse made) according to Table 14. Radioactive labeled amino acid $\left[{ }^{14} \mathrm{C}\right]$ Leu was added separately from the aminoacid mix to estimate the final aa-tRNA yield.

Table 14. In vitro tRNA aminoacylation mix

\begin{tabular}{|c|c|}
\hline Component & $\begin{array}{c}\text { Final } \\
\text { concentration }\end{array}$ \\
\hline TAKM $_{7}$ & $1 \mathrm{x}$ \\
\hline $\mathrm{MgCl}_{2}$ & $13 \mathrm{mM}$ \\
\hline ATP & $3 \mathrm{mM}$ \\
\hline DTT & $2 \mathrm{mM}$ \\
\hline $\begin{array}{c}\text { Mix of aminoacids } \\
\text { (except leucine })\end{array}$ & $0.3 \mathrm{mM}$ \\
\hline$\left[{ }^{14} \mathrm{C}\right]$ Leu & $25 \mu \mathrm{M}$ \\
\hline Total tRNA & $80 \mathrm{OD} / \mathrm{ml}$ \\
\hline S100 & $3 \%$ \\
\hline
\end{tabular}

The preparative in vitro aminoacylation reaction was carried out for 30 min at $37^{\circ} \mathrm{C}$ and stopped by adding $1 / 10 \mathrm{~V} \mathrm{KOAc}(20 \%, \mathrm{pH} 5.0)$ (). The total aa-tRNA was isolated by phenol extraction and precipitated from the aqueous phase overnight with $1 / 10 \mathrm{~V} \mathrm{KOAC}$ $(20 \%, \mathrm{pH} 5.0)$ and $2.5 \mathrm{~V}$ ethanol at $-20^{\circ} \mathrm{C}$. The aa-tRNA was pelleted by centrifugation for $1 \mathrm{~h}$ at $4000 \mathrm{rpm}$ at $4^{\circ} \mathrm{C}$, dissolved in Milli-Q $\mathrm{H}_{2} \mathrm{O}$ purified on a HiTrap Q HP anionexchange column using FPLC. Aa-tRNA fractions were analyzed by UV measurement at $260 \mathrm{~nm}$, united according to elution profile and again precipitated overnight with $1 / 10 \mathrm{~V}$ $\mathrm{KOAc} 20 \%$, $(\mathrm{pH} 5.0)$ and $2.5 \mathrm{~V}$ ethanol at $-20^{\circ} \mathrm{C}$. The total aa-tRNA pellet was pulled down by centrifugation for $1 \mathrm{~h}$ at $4000 \mathrm{rpm}$ at $4^{\circ} \mathrm{C}$ and dissolved in Milli-Q $\mathrm{H}_{2} \mathrm{O}$. The quality of produced total aa-tRNA was checked by spectrophotometric measurement at $260 \mathrm{~nm}$ 
Materials and Methods

and $\left[{ }^{14} \mathrm{C}\right]$ liquid scintillation counting with Ultima Gold ${ }^{\mathrm{TM}} \mathrm{XR}$ scintillation fluid. The ratio between $\left[{ }^{14} \mathrm{C}\right]$ Leu and total tRNA molar concentrations was close to the ones reported in vivo (Dong \& Kurland, 1996).

\subsubsection{Tris-Tricine gel electrophoresis}

Gels were performed using three gel layers with 4\% T, 3\% C for the stacking gel, 10\% $\mathrm{T}$ and $3 \% \mathrm{C}$ for the spacer gel and $16.5 \% \mathrm{~T}$ and $6 \% \mathrm{C}$ for the separating gel; with $\mathrm{T}$ being acrylamide and C being bis-acrylamide (Schägger \& von Jagow, 1987; Schägger, 2006). All gels contained $1 \mathrm{M}$ Tris $\mathrm{pH} 8.45$ and $0.1 \%$ SDS and were polymerized by addition of $1 / 100 \mathrm{~V}$ ammonium persulfate solution (APS) (10\%) and 1/1000 V tetramethylenediamine (TEMED) (100\%). Samples were mixed with $1 / 5 \mathrm{~V} \mathrm{NaOH}(2 \mathrm{M})$, incubated for $30 \mathrm{~min}$ at $37^{\circ} \mathrm{C}$ for peptide chain release and neutralized with $1 / 5 \mathrm{~V}$ HEPES-KOH (2 M, pH 5.0). The samples then were mixed with $1 \mathrm{~V}$ loading buffer and incubated for $10 \mathrm{~min}$ at $70^{\circ} \mathrm{C}$ before loading. PAGE was carried out with cathode and anode running buffers at $30 \mathrm{~V}$ for 30 min followed by $2-4 \mathrm{~h}$ at $120 \mathrm{~V}$.

\subsubsection{Reconstitution of labeled ribosomal subunits}

$\Delta S 6$ and $\Delta L 9$ mutant ribosomal $30 S$ and $50 S$ were prepared as described (Sharma et al., 2016) and provided by the laboratory facility. Labeled proteins Cy5-S6 and Cy3-L9 were prepared as descibed (Sharma et al., 2016) and provided by Heena Sharma. Purified $\Delta S 630 S$ subunits were reconstituted with a 2 -fold excess of labeled protein $S 6$ in $\mathrm{S} 6$ reconstitution buffer for $30 \mathrm{~min}$ at $42^{\circ} \mathrm{C}$. After $30 \mathrm{~min}$ the concentration of $\mathrm{Mg}^{2+}$ was raised to $20 \mathrm{mM}$ by adding $1 \mathrm{M} \mathrm{MgCl}_{2}$ and the reaction mixture was incubated for $30 \mathrm{~min}$ at $42^{\circ} \mathrm{C}$. Purified $\Delta \mathrm{L} 950 \mathrm{~S}$ were reconstituted with a 2 -fold excess of labeled protein $L 9$ in $\mathrm{L} 9$ reconstitution buffer for $1 \mathrm{~h}$ at $37^{\circ} \mathrm{C}$. The reaction mixture was incubated for $15 \mathrm{~min}$ on ice and then centrifuged at $13000 \mathrm{rpm}$ for $15 \mathrm{~min}$. Excess of labeled protein was separated through a $1.1 \mathrm{M}$ sucrose cushion in 1x TAKM 21 buffer in an MLA 130 fixed angle rotor in an ultracentrifuge at $259000 \mathrm{~g}$ at $4^{\circ} \mathrm{C}$ for $3 \mathrm{~h}$. The quantity of purified subunits was checked by spectrophotometric measurements at $260 \mathrm{~nm}$. The $30 \mathrm{~S}$ and $50 \mathrm{~S}$ molar concentration was calculated assuming that $67 \mathrm{pmol}$ of $30 \mathrm{~S}$ and $37 \mathrm{pmol}$ of $50 S$ subunits absorb 1 A260 unit (Richter, 1976). The labeling efficiency was determined 
Materials and Methods

according to molar extinction coefficient of the dye, assuming $150000 \mathrm{~cm}^{-1} \mathrm{M}^{-1}$ for Cy3 and $250000 \mathrm{~cm}^{-1} \mathrm{M}^{-1}$ for Cy5. The labeling efficiency was $90-100 \%$.

\subsubsection{Initiation complex formation (non-labeled ribosomes)}

WT 70 S ribosomes from $E$. coli MRE 600, $\Delta$ L9 ribosomes from a $\Delta$ L9 BW25113 $E$. coli strain and initiation factors (IF1, IF2, IF3) were prepared as described (Rodnina \& Wintermeyer, 1995; Milon et al., 2007; Sharma et al., 2016) and provided by the laboratory facility. Mutant S12 70S ribosomes were prepared as described (Pósfai et al., 2006) and provided by Michael Pearson. Initiation complex (IC) were formed by incubating 70 S ribosomes $(0.5 \mu \mathrm{M})$, mRNA $(2 \mu \mathrm{M})$, IF1, IF2 and IF3 (0.75 $\mu \mathrm{M}$ each), GTP (1 mM), DTT $(1 \mathrm{mM})$ and BODIPY-[ $\left.{ }^{3} \mathrm{H}\right]$ Met-tRNA ${ }^{\mathrm{fMet}}(0.75 \mu \mathrm{M})$ in $1 \mathrm{x}$ TAKM 7 buffer for 45 min at $37^{\circ} \mathrm{C}$. The quality of formed 70 S IC was checked by immobilizing the IC $(10 \mathrm{pmol}$ sample) on a nitrocellulose filter with subsequent buffer washing, filter dissolving in Quickzint 361 scintillation fluid and $\left[{ }^{3} \mathrm{H}\right]$ filter scintillation counting. The initiation efficiency was calculated by the following formula:

$$
\text { 70S IC formation efficiency, } \%=\frac{\left[{ }^{3} \mathrm{H}\right] \text { counts measured, } \mathrm{dpm}}{10 \mathrm{pmol} \times \text { specific activity } \mathrm{f}\left[{ }^{3} \mathrm{H}\right] \mathrm{Met}-\mathrm{tRNA} \mathrm{fMet}^{\mathrm{fm}}, \frac{\mathrm{dpm}}{\mathrm{pmol}}} \times 100 \%
$$

\subsubsection{Initiation complex formation (labeled ribosomes)}

Fluorescence-labeled $30 \mathrm{~S}$ and $50 \mathrm{~S}$ subunits were prepared as described (4.2.6). Initiation complexes with the desired mRNA were prepared as described (4.2.7) using fluorescence-labeled $30 \mathrm{~S}$ subunits $(1.0 \mu \mathrm{M})$ together with a 1.5-fold excess of labeled $50 \mathrm{~S}$ subunits $(0.16 \mu \mathrm{M}), \mathrm{f}\left[{ }^{3} \mathrm{H}\right] \mathrm{Met}-\mathrm{tRNA}{ }^{\mathrm{fMet}}(0.75 \mu \mathrm{M})$ and HiFi buffer. 
Materials and Methods

\subsubsection{Initiation complex purification}

Initiation complexes with desired mRNA (4.2.8) were purified by centrifugation through a $1.1 \mathrm{M}$ sucrose cushion in $1 \mathrm{x}$ TAKM 7 buffer in TLS-55 swinging-bucket rotor in an ultracentrifuge at $55000 \mathrm{rpm}$ at $4^{\circ} \mathrm{C}$ for $2 \mathrm{~h}$. Pellets were dissolved in $1 \times \mathrm{TAKM}_{7}$ buffer. Quality and quantity of purified IC were checked by spectrophotometric measurements at $260 \mathrm{~nm}$ and $\left[{ }^{3} \mathrm{H}\right]$ liquid scintillation counting with Ultima Gold ${ }^{\mathrm{TM}}$ XR scintillation fluid. The $70 S$ ribosomal molar concentration was calcutated assuming that $23 \mathrm{pmol}$ of $70 \mathrm{~S}$ ribosomes absorb $1 \mathrm{~A}_{260}$ unit (Richter, 1976), the f[$\left.{ }^{3} \mathrm{H}\right]$ Met-tRNA ${ }^{f M e t}$ molar concentration was calculated using the specific activity of a particular tRNA. The amount of the fMettRNA ${ }^{\text {fMet }}$ bound to the ribosome was calculated from the ratio $\left[{ }^{3} \mathrm{H}\right]$ radioactivity to $A_{260}$ in the ribosome fraction.

\subsubsection{In vitro translation by monosomes and polysomes}

Initiation complexes with the desired mRNA were prepared as described (4.2.7) using BODIPY- $\left[{ }^{3} \mathrm{H}\right]$ Met-tRNA ${ }^{\mathrm{fMet}}(0.75 \mu \mathrm{M})$ and HiFi buffer. EF-Tu and EF-G were prepared as described (Rodnina \& Wintermeyer, 1994; Rodnina et al., 1999) and provided by the laboratory facility. Domain IV EF-G mutants were prepared as described (Peng et al., 2019) and provided by Bee-Zen Peng. The ternary complex EF-Tu-GTP-aminoacyltRNA was prepared by incubating EF-Tu $(58 \mu \mathrm{M})$ with GTP $(1 \mathrm{mM})$, DTT (1mM), phosphoenol pyruvate $(3 \mathrm{mM})$ and pyruvate kinase $(0.1 \mathrm{mg} / \mathrm{mL})$ for $15 \mathrm{~min}$ at $37^{\circ} \mathrm{C}$, then adding purified total aa-tRNA $(60 \mu \mathrm{M})$ and EF-G $(2 \mu \mathrm{M})$ and incubating for $1 \mathrm{~min}$ at $37^{\circ} \mathrm{C}$. In vitro translation by monosomes was started by mixing IC (to final concentration 0.16 $\mu \mathrm{M})$ with ternary complexes $(50 \mu \mathrm{M})$ formed with total aminoacyl-tRNA. Translation was carried out at $37^{\circ} \mathrm{C}$ for different time intervals from $3 \mathrm{~s}$ to $1200 \mathrm{~s}$. To form polysomes, translation was carried out in the presence of additionally $30 \mathrm{~S}$ ribosomal subunits $(0.16$ $\mu \mathrm{M}$; 10-fold over the mRNA), 50S ribosomal subunits $(0.24 \mu \mathrm{M})$, IF1, IF2 and IF3 (0.24 $\mu \mathrm{M}$ each) and BODIPY-[3H]Met-tRNA ${ }^{\text {fMet }}(0.24 \mu \mathrm{M})$ to allow re-initiation on the same mRNA. Translation products were separated by Tris-Tricine gel electrophoresis (4.2.5). Fluorescent peptides were detected in gels using Starion IR/FLA-9000 scanner and quantified using the MultiGauge software. Bypassing efficiency was calculated as a ratio of the density corresponding to the byp band to the sum of the byp and stop bands. 
Materials and Methods

\subsubsection{In vitro translation at the different temperatures}

Initiation complexes with desired mRNA were prepared as described (4.2.7) using BODIPY- $\left.{ }^{3} \mathrm{H}\right]$ Met-tRNA ${ }^{\mathrm{fMet}}(0.75 \mu \mathrm{M})$ and HiFi buffer. The ternary complexes were prepared as described (4.2.10). In vitro translation was started by mixing initiation ribosome complexes $(0.08 \mu \mathrm{M})$ with ternary complexes $(50 \mu \mathrm{M})$ with total aminoacyl-tRNA and incubated at temperature range $10-37^{\circ} \mathrm{C}$ for $20 \mathrm{~min}$. Products were separated by Tris-Tricine gel electrophoresis (4.2.5). Fluorescent peptides were detected after gel electrophoresis using Starion IR/FLA-9000 scanner and quantified using the Multi Gauge software. Bypassing efficiency was calculated as a ratio of the density corresponding to the byp band to the sum of the byp and stop bands.

\subsubsection{Stalled take-off complexes}

Translation mixtures containing $70 S$ ribosomes or fluorescence-labeled 305 subunits together with a 1.5- fold excess of labeled 50 S subunits $(0.16 \mu \mathrm{M})(4.2 .7$ or 4.2 .8$)$ and ternary complexes (4.2.10) were incubated for $20 \mathrm{~min}$ at $10^{\circ} \mathrm{C}$. Depending on desired concentraction the resulting ribosome complexes were purified either by gel filtration on BioSuite $450 \mathrm{HR} 8-\mu \mathrm{m}$ column at $4^{\circ} \mathrm{C}$ or by centrifugation through a $1.1 \mathrm{M}$ sucrose cushion in HiFi buffer in TLA-100 fixed-angle rotor in an ultracentrifuge at $68000 \mathrm{rpm}$ at $4^{\circ} \mathrm{C}$ for $1 \mathrm{~h}$; pellets were dissolved in HiFi buffer. The amount of the nascent peptide bound to the ribosome was calculated from the ratio f[ $\left.{ }^{3} \mathrm{H}\right] \mathrm{Met} \mathrm{TRNA} \mathrm{A}^{\mathrm{fMet}}$ radioactivity to $\mathrm{A}_{260}$ in the ribosome fraction.

\subsubsection{Leu incorporation into the nascent peptide}

Stalled take-off complexes $(0.1 \mu \mathrm{M})$ (4.2.12) were mixed with ternary complexes prepared as described (4.2.10) with EF-Tu-GTP- $\left[{ }^{14} \mathrm{C}\right]$ Leu-tRNA ${ }^{\text {Leu }}$ UUA $(0.3 \mu \mathrm{M})$. After incubation for $2 \mathrm{~min}$ at $37^{\circ} \mathrm{C}$, samples were quenched with $1 / 2 \mathrm{~V} \mathrm{KOH}(1 \mathrm{M})$ and hydrolyzed for $30 \mathrm{~min}$ at $37^{\circ} \mathrm{C}$. After neutralization with $1 / 10 \mathrm{~V} \mathrm{CH}_{3} \mathrm{COOH}(100 \%)$, the products were analyzed by high-performance liquid chromatography (HPLC) using an adapted gradient of acetonitrile in $0.1 \%$ trifluoroacetic acid on a LiChrospher 100 RP-8 column. 


\subsubsection{The GTP hydrolysis assay}

The assay was performed by mixing stalled take-off complexes $(0.1 \mu \mathrm{M})(4.2 .12)$ with EF-G $(0.1 \mu \mathrm{M})$ and GTP $(10 \mu \mathrm{M})$ containing trace amounts of $\left[\gamma^{-32} \mathrm{P}\right] \mathrm{GTP}$. Reactions were incubated for different time intervals from $10 \mathrm{~s}$ to $1200 \mathrm{~s}$ at $37^{\circ} \mathrm{C}$, and aliquots were taken at indicated time intervals and quenched with $1 \mathrm{~V}$ formic acid (50\%). Samples were analyzed by thin-layer chromatography using a $0.5 \mathrm{M} \mathrm{KH}_{2} \mathrm{PO}_{4}(\mathrm{pH} 3.5)$ running buffer. Radioactivity was detected using a phosphoimager system. GTP consumption during bypassing was calculated as a difference of GTP hydrolysis rounds between bypassing ribosomes and ribosomes stalled on an mRNA truncated downstream of the stop codon. The extent of bypassing was measured in parallel using the $\left[{ }^{14} \mathrm{C}\right]$ Leu incorporation assay perfomed as described (4.2.13) using EF-G $(0.1 \mu \mathrm{M})$ and GTP $(10 \mu \mathrm{M})$. For normalization we used the ribosomal fraction active in bypassing according to $\left[{ }^{14} \mathrm{C}\right]$ Leu incorporation assay ( 20\%).

\subsubsection{Time resolved puromycin assay}

Pretranslocation complexes (with WT or mutant ribosomes) carrying tRNA ${ }^{\text {fMet }}$ in the $P$ site and $f\left[{ }^{3} \mathrm{H}\right]$ Met- $\left[{ }^{14} \mathrm{C}\right]$ Phe-tRNA ${ }^{\text {Phe }}$ in the A site were prepared as described (Cunha et al., 2013). Pretranslocation complexes $(0.1 \mu \mathrm{M})$ were incubated with a catalytic amount of EF-G (1 nM) or without EF-G in 1x TAKM7 buffer. Samples were taken and reacted with puromycin $(1 \mathrm{mM})$ for $10 \mathrm{~s}$ before being quenched with $1.5 \mathrm{M}$ sodium acetate $(\mathrm{pH}$ 4.5) saturated with $\mathrm{MgSO}_{4}$. The peptide products were extracted with ethyl acetate and quantified by radioactivity counting. The experiment was performed and analyzed by BeeZen Peng. 
Materials and Methods

\subsubsection{Single-round translocation assay}

Pretranslocation complexes (with WT or mutant ribosomes) were prepared as described (4.2.15) with an Alexa Fluor 405-labeled mRNA (mMF14Alx405). Complexes $(0.05 \mu \mathrm{M})$ were rapidly mixed with EF-G $(4 \mu \mathrm{M})$ in a stopped-flow apparatus at $37^{\circ} \mathrm{C}$. The dye was excited at $400 \mathrm{~nm}$, and fluorescence was measured after passing a KV418 cutoff filter (Schott). The experiment was performed and analyzed by Bee-Zen Peng.

\subsubsection{7. tRNA stability assay}

Pretranslocation complexes (with WT or mutant ribosomes) carrying tRNA ${ }^{\mathrm{fMet}}$ in the $P$ site and $f\left[{ }^{3} \mathrm{H}\right]$ Met- $\left[{ }^{[4} \mathrm{C}\right]$ Phe-tRNA ${ }^{\text {Phe }}$ in the $A$ site were prepared as described (Cunha et al., 2013) and incubated in $1 \times \mathrm{TAKM}_{7}$ buffer at $37^{\circ} \mathrm{C}$. tRNA binding was assayed by nitrocellulose filtration of the complexes, and the amount of bound tRNA was quantified by radioactive counting. The experiment was performed and analyzed by Bee-Zen Peng.

\subsubsection{Single-molecule experiments using TIRF microscopy}

The stalled ribosome complexes prepared as described (4.2.12) using labeled ribosomes (4.2.8) were diluted in HiFi buffer to a final concentration of $0.5 \mathrm{nM}$ and immobilized on biotin-polyethylene glycol quartz slides pre-incubated with NeutrAvidin using the mRNA annealed to a biotinylated primer. The imaging buffer was prepared as described (Adio et al., 2015). smFRET experiments were performed at $22^{\circ} \mathrm{C}$ or $4^{\circ}$ to $10^{\circ} \mathrm{C}$ temperature on an IX81 inverted objective based TIRF microscope with a $100 \times 1.45$ numerical aperture oil immersion objective. Images were recorded at a time resolution of $30 \mathrm{frames} / \mathrm{s}$. To image complexes at low temperature an aluminum alloy cube cooled on ice and placed on the microscope slide was used during the measurements. The temperature was controlled and maintained constant within approximately $1^{\circ} \mathrm{C}$. Fluorescence time traces for donor (Су3) and acceptor (Сy5) were extracted and analyzed using custom-made MATLAB (MathWorks) software as described (Adio et al., 2015). The distribution of FRET states shown in the state histograms fit to a sum of Gaussian functions using a nonlinear minimization procedure (fminsearch, MATLAB). The $\mathrm{R}^{2}$ value for all fits was larger than 0.98 . For normalization we estimated that about $40 \%$ of complexes change the conformation from the non-rotated to either rotated or 
hyper-rotated under the conditions of the smFRET experiment, consistent with the bypassing efficiency ( $40 \%$ ) upon labeling the ribosomes, whereas the remaining fraction remains in the non-rotated state and was omitted from the calculations. Imaging and analysis of smFRET data was carried out by Tamara Senyushkina. 
References

\section{References}

Adio, S., Senyushkina, T., Peske, F., Fischer, N., Wintermeyer, W., \& Rodnina, M. V. (2015). Fluctuations between multiple EF-G-induced chimeric tRNA states during translocation on the ribosome. Nature Communications. https://doi.org/10.1038/ncomms8442

Adio, S., Sharma, H., Senyushkina, T., Karki, P., Maracci, C., Wohlgemuth, I., ... Rodnina, M. V. (2018). Dynamics of ribosomes and release factors during translation termination in E. Coli. ELife. https://doi.org/10.7554/eLife.34252

Agirrezabala, X., \& Frank, J. (2010). From DNA to proteins via the ribosome: structural insights into the workings of the translation machinery. Human Genomics, 4(4), 226-237. https://doi.org/10.1186/1479-7364-4-4-226

Agirrezabala, X., Samatova, E., Klimova, M., Zamora, M., Gil-Carton, D., Rodnina, M. V., \& Valle, M. (2017). Ribosome rearrangements at the onset of translational bypassing. Science Advances, 3(6), 1-9. https://doi.org/10.1126/sciadv.1700147

Aitken, C. E., \& Lorsch, J. R. (2012). A mechanistic overview of translation initiation in eukaryotes. Nature Structural and Molecular Biology. https://doi.org/10.1038/nsmb.2303

Alkalaeva, E. Z., Pisarev, A. V., Frolova, L. Y., Kisselev, L. L., \& Pestova, T. V. (2006). In Vitro Reconstitution of Eukaryotic Translation Reveals Cooperativity between Release Factors eRF1 and eRF3. Cell. https://doi.org/10.1016/j.cell.2006.04.035

Andreeva, I., Belardinelli, R., \& Rodnina, M. V. (2018). Translation initiation in bacterial polysomes through ribosome loading on a standby site on a highly translated mRNA. Proceedings of the National Academy of Sciences of the United States of America. https://doi.org/10.1073/pnas.1718029115

Atkins, J. F., Loughran, G., Bhatt, P. R., Firth, A. E., \& Baranov, P. V. (2016). Ribosomal frameshifting and transcriptional slippage: From genetic steganography and cryptography to adventitious use. Nucleic Acids Research. https://doi.org/10.1093/nar/gkw530

Ban, N., Nissen, P., Hansen, J., Moore, P. B., \& Steitz, T. A. (2000). The complete atomic structure of the large ribosomal subunit at $2.4 \AA$ resolution. Science. https://doi.org/10.1126/science.289.5481.905

Baranov, P. V., Gesteland, R. F., \& Atkins, J. F. (2002). Recoding: Translational bifurcations in gene expression. Gene. https://doi.org/10.1016/S03781119(02)00423-7

Belardinelli, R., \& Rodnina, M. V. (2017). Effect of Fusidic Acid on the Kinetics of Molecular Motions During EF-G-Induced Translocation on the Ribosome. Scientific Reports. https://doi.org/10.1038/s41598-017-10916-8

Belcourt, M. F., \& Farabaugh, P. J. (1990). Ribosomal frameshifting in the yeast retrotransposon Ty: tRNAs induce slippage on a 7 nucleotide minimal site. Cell. 
https://doi.org/10.1016/0092-8674(90)90371-K

Belew, A. T., Meskauskas, A., Musalgaonkar, S., Advani, V. M., Sulima, S. O., Kasprzak, W. K., ... Dinman, J. D. (2014). Ribosomal frameshifting in the CCR5 mRNA is regulated by miRNAs and the NMD pathway. Nature. https://doi.org/10.1038/nature13429

Beringer, M., Bruell, C., Xiong, L., Pfister, P., Bieling, P., Katunin, V. I., ... Rodnina, M. V. (2005). Essential mechanisms in the catalysis of peptide bond formation on the ribosome. Journal of Biological Chemistry. https://doi.org/10.1074/jbc.M507961200

Bieling, P., Beringer, M., Adio, S., \& Rodnina, M. V. (2006). Peptide bond formation does not involve acid-base catalysis by ribosomal residues. Nature Structural and Molecular Biology. https://doi.org/10.1038/nsmb1091

Bieri, P., Greber, B. J., \& Ban, N. (2018). High-resolution structures of mitochondrial ribosomes and their functional implications. Current Opinion in Structural Biology, 49, 44-53. https://doi.org/10.1016/j.sbi.2017.12.009

Blanchet, S., Cornu, D., Argentini, M., \& Namy, O. (2014). New insights into the incorporation of natural suppressor tRNAs at stop codons in Saccharomyces cerevisiae. Nucleic Acids Research, 42(15), 10061-10072. https://doi.org/10.1093/nar/gku663

Bock, L. V., Blau, C., Schröder, G. F., Davydov, I. I., Fischer, N., Stark, H., ... Grubmüller, H. (2013). Energy barriers and driving forces in tRNA translocation through the ribosome. Nature Structural and Molecular Biology, 20(12), 1390-1396. https://doi.org/10.1038/nsmb.2690

Bock, L. V., Caliskan, N., Korniy, N., Peske, F., Rodnina, M. V., \& Grubmüller, H. (2019). Thermodynamic control of -1 programmed ribosomal frameshifting. Nature Communications, 10(1). https://doi.org/10.1038/s41467-019-12648-x

Bonocora, R. P., Zeng, Q., Abel, E. V., \& Shub, D. A. (2011). A homing endonuclease and the 50 -nt ribosomal bypass sequence of phage T4 constitute a mobile DNA cassette. Proceedings of the National Academy of Sciences of the United States of America, 108(39), 16351-16356. https://doi.org/10.1073/pnas.1107633108

Bourne, H. R., Sanders, D. A., \& McCormick, F. (1991). The GTPase superfamily: conserved structure and molecular mechanism. Nature, 349(6305), 117-127. https://doi.org/10.1038/349117a0

Brandt, F., Etchells, S. A., Ortiz, J. O., Elcock, A. H., Hartl, F. U., \& Baumeister, W. (2009). The Native 3D Organization of Bacterial Polysomes. Cell, 136(2), 261-271. https://doi.org/10.1016/j.cell.2008.11.016

Brierley, I., Digard, P., \& Inglis, S. C. (1989). Characterization of an efficient coronavirus ribosomal frameshifting signal: Requirement for an RNA pseudoknot. Cell. https://doi.org/10.1016/0092-8674(89)90124-4

Brierley, I., \& Dos Ramos, F. J. (2006). Programmed ribosomal frameshifting in HIV-1 and the SARS-CoV. Virus Research. https://doi.org/10.1016/j.virusres.2005.10.008 
References

Brilot, A. F., Korostelev, A. A., Ermolenko, D. N., \& Grigorieff, N. (2013). Structure of the ribosome with elongation factor $\mathrm{G}$ trapped in the pretranslocation state.

Proceedings of the National Academy of Sciences of the United States of America. https://doi.org/10.1073/pnas.1311423110

Bucklin, D. J., Wills, N. M., Gesteland, R. F., \& Atkins, J. F. (2005). P-site pairing subtleties revealed by the effects of different tRNAs on programmed translational bypassing where anticodon re-pairing to mRNA is separated from dissociation. Journal of Molecular Biology, 345(1), 39-49. https://doi.org/10.1016/j.jmb.2004.10.037

Caliskan, N., Katunin, V. I., Belardinelli, R., Peske, F., \& Rodnina, M. V. (2014). Programmed -1 frameshifting by kinetic partitioning during impeded translocation. Cell. https://doi.org/10.1016/j.cell.2014.04.041

Caliskan, N., Wohlgemuth, I., Korniy, N., Pearson, M., Peske, F., \& Rodnina, M. V. (2017). Conditional Switch between Frameshifting Regimes upon Translation of dnaX mRNA. Molecular Cell. https://doi.org/10.1016/j.molcel.2017.04.023

Chang, B., Halgamuge, S., \& Tang, S. L. (2006). Analysis of SD sequences in completed microbial genomes: Non-SD-led genes are as common as SD-led genes. Gene. https://doi.org/10.1016/j.gene.2006.01.033

Charneski, C. A., \& Hurst, L. D. (2013). Positively Charged Residues Are the Major Determinants of Ribosomal Velocity. PLoS Biology, 11(3). https://doi.org/10.1371/journal.pbio.1001508

Chen, C., Cui, X., Beausang, J. F., Zhang, H., Farrell, I., Cooperman, B. S., \& Goldman, Y. E. (2016). Elongation factor $G$ initiates translocation through a power stroke. Proceedings of the National Academy of Sciences of the United States of America. https://doi.org/10.1073/pnas.1602668113

Chen, C., Zhang, H., Broitman, S. L., Reiche, M., Farrell, I., Cooperman, B. S., \& Goldman, Y. E. (2013). Dynamics of translation by single ribosomes through mRNA secondary structures. Nature Structural and Molecular Biology, 20(5), 582-588. https://doi.org/10.1038/nsmb.2544

Chen, J., Coakley, A., O'Connor, M., Petrov, A., O'Leary, S. E., Atkins, J. F., \& Puglisi, J. D. (2015). Coupling of mRNA Structure Rearrangement to Ribosome Movement during Bypassing of Non-coding Regions. Cell. https://doi.org/10.1016/j.cell.2015.10.064

Chen, J., Petrov, A., Johansson, M., Tsai, A., O'leary, S. E., \& Puglisi, J. D. (2014). Dynamic pathways of -1 translational frameshifting. Nature, 512(7514), 328-332. https://doi.org/10.1038/nature13428

Chevance, F. F. V., Le Guyon, S., \& Hughes, K. T. (2014). The Effects of Codon Context on In Vivo Translation Speed. PLoS Genetics. https://doi.org/10.1371/journal.pgen.1004392

Chiba, S., Lamsa, A., \& Pogliano, K. (2009). A ribosome-nascent chain sensor of 
membrane protein biogenesis in Bacillus subtilis. EMBO Journal. https://doi.org/10.1038/emboj.2009.280

Cornish, P. V., Ermolenko, D. N., Noller, H. F., \& Ha, T. (2008). Spontaneous Intersubunit Rotation in Single Ribosomes. Molecular Cell, 30(5), 578-588. https://doi.org/10.1016/j.molcel.2008.05.004

Cornish, P. V., Ermolenko, D. N., Staple, D. W., Hoang, L., Hickerson, R. P., Noller, H. F., \& Ha, T. (2009). Following movement of the L1 stalk between three functional states in single ribosomes. Proceedings of the National Academy of Sciences of the United States of America. https://doi.org/10.1073/pnas.0813180106

Crick, F. H. C. (1966). Codon-anticodon pairing: The wobble hypothesis. Journal of Molecular Biology. https://doi.org/10.1016/S0022-2836(66)80022-0

Cukras, A. R., Southworth, D. R., Brunelle, J. L., Culver, G. M., \& Green, R. (2003). Ribosomal proteins $\mathrm{S} 12$ and $\mathrm{S} 13$ function as control elements for translocation of the mRNA:tRNA complex. Molecular Cell, 12(2), 321-328. https://doi.org/10.1016/S1097-2765(03)00275-2

Cunha, C. E., Belardinelli, R., Peske, F., Holtkamp, W., Wintermeyer, W., \& Rodnina, M. V. (2013). Dual use of GTP hydrolysis by elongation factor $\mathrm{G}$ on the ribosome. Translation. https://doi.org/10.4161/trla.24315

Dana, A., \& Tuller, T. (2014). The effect of tRNA levels on decoding times of mRNA codons. Nucleic Acids Research. https://doi.org/10.1093/nar/gku646

Dao Duc, K., \& Song, Y. S. (2018). The impact of ribosomal interference, codon usage, and exit tunnel interactions on translation elongation rate variation. PLOS Genetics. https://doi.org/10.1371/journal.pgen.1007166

Dever, T. E., \& Green, R. (2012). The elongation, termination, and recycling phases of translation in eukaryotes. Cold Spring Harbor Perspectives in Biology. https://doi.org/10.1101/cshperspect.a013706

Diaconu, M., Kothe, U., Schlünzen, F., Fischer, N., Harms, J. M., Tonevitsky, A. G., ... Wahl, M. C. (2005). Structural basis for the function of the ribosomal L7/12 stalk in factor binding and GTpase activation. Cell, 121(7), 991-1004. https://doi.org/10.1016/j.cell.2005.04.015

Doerfel, L. K., Wohlgemuth, I., Kothe, C., Peske, F., Urlaub, H., \& Rodnina, M. V. (2013). EF-P is essential for rapid synthesis of proteins containing consecutive proline residues. Science. https://doi.org/10.1126/science.1229017

Doerfel, L. K., Wohlgemuth, I., Kubyshkin, V., Starosta, A. L., Wilson, D. N., Budisa, N., \& Rodnina, M. V. (2015). Entropic contribution of elongation factor $P$ to proline positioning at the catalytic center of the ribosome. Journal of the American Chemical Society. https://doi.org/10.1021/jacs.5b07427

Dong, H., Nilsson, L., \& Kurland, C. G. (1996). Co-variation of tRNA abundance and codon usage in Escherichia coli at different growth rates. Journal of Molecular Biology. https://doi.org/10.1006/jmbi.1996.0428 
References

Dong, J., Nanda, J. S., Rahman, H., Pruitt, M. R., Shin, B. S., Wong, C. M., ... Hinnebusch, A. G. (2008). Genetic identification of yeast 18S rRNA residues required for efficient recruitment of initiator tRNAMet and AUG selection. Genes and Development. https://doi.org/10.1101/gad.1696608

Dunkle, J. A., \& Cate, J. H. D. (2011). The packing of ribosomes in crystals and polysomes. In Ribosomes. https://doi.org/10.1007/978-3-7091-0215-2_6

Dunkle, J. A., Wang, L., Feldman, M. B., Pulk, A., Chen, V. B., Kapral, G. J., ... Cate, J. H. D. (2011). Structures of the bacterial ribosome in classical and hybrid states of tRNA binding. Science. https://doi.org/10.1126/science.1202692

Ermolenko, D. N., Majumdar, Z. K., Hickerson, R. P., Spiegel, P. C., Clegg, R. M., \& Noller, H. F. (2007). Observation of Intersubunit Movement of the Ribosome in Solution Using FRET. Journal of Molecular Biology. https://doi.org/10.1016/j.jmb.2007.04.042

Firth, A. E., Wills, N. M., Gesteland, R. F., \& Atkins, J. F. (2011). Stimulation of stop codon readthrough: Frequent presence of an extended 3' RNA structural element. Nucleic Acids Research. https://doi.org/10.1093/nar/gkr224

Fischer, N., Konevega, A. L., Wintermeyer, W., Rodnina, M. V., \& Stark, H. (2010). Ribosome dynamics and tRNA movement by time-resolved electron cryomicroscopy. Nature. https://doi.org/10.1038/nature09206

Fischer, N., Neumann, P., Konevega, A. L., Bock, L. V., Ficner, R., Rodnina, M. V., \& Stark, H. (2015). Structure of the E. coli ribosome-EF-Tu complex at $<3 \AA$ resolution by Cs-corrected cryo-EM. Nature, 520(7548), 567-570. https://doi.org/10.1038/nature14275

Frolova, L., Goff, X. L. E., Zhouravleva, G., Davydova, E., Philippe, M., \& Kisselev, L. (1996). Eukaryotic polypeptide chain release factor eRF3 is an eRF1- and ribosome-dependent guanosine triphosphatase. RNA.

Frolova, L. Y., Tsivkovskii, R. Y., Sivolobova, G. F., Oparina, N. Y., Serpinsky, O. I., Blinov, V. M., ... Kisselev, L. L. (1999). Mutations in the highly conserved GGQ motif of class I polypeptide release factors abolish ability of human eRF1 to trigger peptidyl-tRNA hydrolysis. RNA. https://doi.org/10.1017/S135583829999043X

Gallant, J. A., \& Lindsley, D. (1992). Leftward ribosome frameshifting at a hungry codon. Journal of Molecular Biology. https://doi.org/10.1016/0022-2836(92)90713-T

Gao, N., Zavialov, A. V., Li, W., Sengupta, J., Valle, M., Gursky, R. P., ... Frank, J. (2005). Mechanism for the disassembly of the posttermination complex inferred from Cryo-EM studies. Molecular Cell, 18(6), 663-674. https://doi.org/10.1016/j.molcel.2005.05.005

Garofalo, R., Wohlgemuth, I., Pearson, M., Lenz, C., Urlaub, H., \& Rodnina, M. V. (2019). Broad range of missense error frequencies in cellular proteins. Nucleic Acids Research. https://doi.org/10.1093/nar/gky1319

Gong, F., \& Yanofsky, C. (2002). Instruction of translating ribosome by nascent peptide. 
Science, 297(5588), 1864-1867. https://doi.org/10.1126/science.1073997

Guo, Z., \& Noller, H. F. (2012). Rotation of the head of the 30 S ribosomal subunit during mRNA translocation. Proceedings of the National Academy of Sciences of the United States of America, 109(50), 20391-20394. https://doi.org/10.1073/pnas.1218999109

Gupta, P., Liu, B., Klepacki, D., Gupta, V., Schulten, K., Mankin, A. S., \& VázquezLaslop, N. (2016). Nascent peptide assists the ribosome in recognizing chemically distinct small molecules. Nature Chemical Biology. https://doi.org/10.1038/nchembio.1998

Gurevich, V. V., Pokrovskaya, I. D., Obukhova, T. A., \& Zozulya, S. A. (1991). Preparative in vitro mRNA synthesis using SP6 and T7 RNA polymerases. Analytical Biochemistry, 195(2), 207-213. https://doi.org/10.1016/00032697(91)90318-N

Gurvich, O. L., Baranov, P. V., Zhou, J., Hammer, A. W., Gesteland, R. F., \& Atkins, J. F. (2003). Sequences that direct significant levels of frameshifting are frequent in coding regions of Escherichia coli. EMBO Journal. https://doi.org/10.1093/emboj/cdg561

Guydosh, N. R., \& Green, R. (2014). Dom34 rescues ribosomes in 3' untranslated regions. Cell. https://doi.org/10.1016/j.cell.2014.02.006

Hellen, C.U.T. (2018). Translation termination and ribosome recycling in eukaryotes. Cold Spring Harb Perspect Biol 10.

Herbst, K. L., Nichols, L. M., Gesteland, R. F., \& Weiss, R. B. (1994). A mutation in ribosomal protein $L 9$ affects ribosomal hopping during translation of gene 60 from bacteriophage T4. Proceedings of the National Academy of Sciences of the United States of America, 91(26), 12525-12529. https://doi.org/10.1073/pnas.91.26.12525

Herr, A. J., Atkins, J. F., \& Gesteland, R. F. (2000). Coupling of Open Reading Frames by Translational Bypassing. Annual Review of Biochemistry. https://doi.org/10.1146/annurev.biochem.69.1.343

Herr, A. J., Nelson, C. C., Wills, N. M., Gesteland, R. F., \& Atkins, J. F. (2001). Analysis of the roles of tRNA structure, ribosomal protein L9, and the bacteriophage T4 gene 60 bypassing signals during ribosome slippage on mRNA. Journal of Molecular Biology, 309(5), 1029-1048. https://doi.org/10.1006/jmbi.2001.4717

Herr, A. J., Wills, N. M., Nelson, C. C., Gesteland, R. F., \& Atkins, J. F. (2001). Drop-off during ribosome hopping. Journal of Molecular Biology, 311(3), 445-452. https://doi.org/10.1006/jmbi.2001.4899

Hoffman, D. W., Davies, C., Gerchman, S. E., Kycia, J. H., Porter, S. J., White, S. W., \& Ramakrishnan, V. (1994). Crystal structure of prokaryotic ribosomal protein L9: a bi-lobed RNA-binding protein. The EMBO Journal. https://doi.org/10.1002/j.14602075.1994.tb06250.x

Holtkamp, W., Wintermeyer, W., \& Rodnina, M. V. (2014). Synchronous tRNA 
References

movements during translocation on the ribosome are orchestrated by elongation factor G and GTP hydrolysis. BioEssays, 36(10), 908-918.

https://doi.org/10.1002/bies.201400076

Huang, W. M., Ao, S. Z., Casjens, S., Orlandi, R., Zeikus, R., Weiss, R., ... Fang, M. (1988). A persistent untranslated sequence within bacteriophage T4 DNA topoisomerase gene 60. Science. https://doi.org/10.1126/science.2830666

Hussmann, J. A., Patchett, S., Johnson, A., Sawyer, S., \& Press, W. H. (2015). Understanding Biases in Ribosome Profiling Experiments Reveals Signatures of Translation Dynamics in Yeast. PLoS Genetics. https://doi.org/10.1371/journal.pgen.1005732

Ingolia, N. T., Ghaemmaghami, S., Newman, J. R. S., \& Weissman, J. S. (2009). Genome-wide analysis in vivo of translation with nucleotide resolution using ribosome profiling. Science, 324(5924), 218-223. https://doi.org/10.1126/science.1168978

Ito-Harashima, S., Kuroha, K., Tatematsu, T., \& Inada, T. (2007). Translation of the poly $(A)$ tail plays crucial roles in nonstop mRNA surveillance via translation repression and protein destabilization by proteasome in yeast. Genes and Development, 21(5), 519-524. https://doi.org/10.1101/gad.1490207

Ito, K., \& Chiba, S. (2013). Arrest Peptides: Cis -Acting Modulators of Translation . Annual Review of Biochemistry, 82(1), 171-202. https://doi.org/10.1146/annurevbiochem-080211-105026

Jenner, L. B., Demeshkina, N., Yusupova, G., \& Yusupov, M. (2010). Structural aspects of messenger RNA reading frame maintenance by the ribosome. Nature Structural and Molecular Biology, 17(5), 555-560. https://doi.org/10.1038/nsmb.1790

Kavran, J. M., \& Steitz, T. A. (2007). Structure of the Base of the L7/L12 Stalk of the Haloarcula marismortui Large Ribosomal Subunit: Analysis of L11 Movements. Journal of Molecular Biology, 371(4), 1047-1059. https://doi.org/10.1016/j.jmb.2007.05.091

Kima, H. K., Liua, F., Fei, J., Bustamante, C., Gonzalez, R. L., \& Tinoco, I. (2014). A frameshifting stimulatory stem loop destabilizes the hybrid state and impedes ribosomal translocation. Proceedings of the National Academy of Sciences of the United States of America. https://doi.org/10.1073/pnas.1403457111

Komar, A. A. (2009). A pause for thought along the co-translational folding pathway. Trends in Biochemical Sciences. https://doi.org/10.1016/j.tibs.2008.10.002

Komar, A. A. (2016). The Yin and Yang of codon usage. Human Molecular Genetics. https://doi.org/10.1093/hmg/ddw207

Komar, A. A., Lesnik, T., \& Reiss, C. (1999). Synonymous codon substitutions affect ribosome traffic and protein folding during in vitro translation. FEBS Letters. https://doi.org/10.1016/S0014-5793(99)01566-5

Konevega, A. L., Fischer, N., Semenkov, Y. P., Stark, H., Wintermeyer, W., \& Rodnina, 
M. V. (2007). Spontaneous reverse movement of mRNA-bound tRNA through the ribosome. Nature Structural and Molecular Biology.

https://doi.org/10.1038/nsmb1221

Korniy, N., Goyal, A., Hoffmann, M., Samatova, E., Peske, F., Pöhlmann, S., \& Rodnina, M. V. (2019). Modulation of HIV-1 Gag/Gag-Pol frameshifting by tRNA abundance. Nucleic Acids Research. https://doi.org/10.1093/nar/gkz202

Korniy, N., Samatova, E., Anokhina, M. M., Peske, F., \& Rodnina, M. V. (2019). Mechanisms and biomedical implications of -1 programmed ribosome frameshifting on viral and bacterial mRNAs. FEBS Letters. https://doi.org/10.1002/18733468.13478

Korostelev, A., Asahara, H., Lancaster, L., Laurberg, M., Hirschi, A., Zhu, J., ... Noller, H. F. (2008). Crystal structure of a translation termination complex formed with release factor RF2. Proceedings of the National Academy of Sciences of the United States of America. https://doi.org/10.1073/pnas.0810953105

Korostelev, A., Zhu, J., Asahara, H., \& Noller, H. F. (2010). Recognition of the amber UAG stop codon by release factor RF1. EMBO Journal. https://doi.org/10.1038/emboj.2010.139

Kozak, M. (1987). An analysis of 5'-noncoding sequences from 699 vertebrate messenger rNAS. Nucleic Acids Research. https://doi.org/10.1093/nar/15.20.8125

Lang, B. F., Jakubkova, M., Hegedusova, E., Daoud, R., Forget, L., Brejova, B., ... Nosek, J. (2014). Massive programmed translational jumping in mitochondria. Proceedings of the National Academy of Sciences of the United States of America, 111(16), 5926-5931. https://doi.org/10.1073/pnas.1322190111

Laurberg, M., Asahara, H., Korostelev, A., Zhu, J., Trakhanov, S., \& Noller, H. F. (2008). Structural basis for translation termination on the $70 \mathrm{~S}$ ribosome. Nature. https://doi.org/10.1038/nature07115

Li, G., \& Rice, C. M. (1993). The signal for translational readthrough of a UGA codon in Sindbis virus RNA involves a single cytidine residue immediately downstream of the termination codon. Journal of Virology.

Li, G. W., Burkhardt, D., Gross, C., \& Weissman, J. S. (2014). Quantifying absolute protein synthesis rates reveals principles underlying allocation of cellular resources. Cell, 157(3), 624-635. https://doi.org/10.1016/j.cell.2014.02.033

Licznar, P., Mejlhede, N., Prère, M. F., Wills, N., Gesteland, R. F., Atkins, J. F., \& Fayet, O. (2003). Programmed translational -1 frameshifting on hexanucleotide motifs and the wobble properties of tRNAs. EMBO Journal. https://doi.org/10.1093/emboj/cdg465

Lillemoen, J., Cameron, C. S., \& Hoffman, D. W. (1997). The stability and dynamics of ribosomal protein L9: Investigations of a molecular strut by amide proton exchange and circular dichroism. Journal of Molecular Biology.

https://doi.org/10.1006/jmbi.1997.0982 
References

Lin, J., Gagnon, M. G., Bulkley, D., \& Steitz, T. A. (2015). Conformational changes of elongation factor $g$ on the ribosome during tRNA translocation. Cell, 160(1-2), 219227. https://doi.org/10.1016/j.cell.2014.11.049

Liu, W., Chen, C., Kavaliauskas, D., Knudsen, C. R., Goldman, Y. E., \& Cooperman, B. S. (2015). EF-Tu dynamics during pre-translocation complex formation: EFTu.GDP exits the ribosome via two different pathways. Nucleic Acids Research. https://doi.org/10.1093/nar/gkv856

Loughran, G., Chou, M. Y., Ivanov, I. P., Jungreis, I., Kellis, M., Kiran, A. M., ... Atkins, J. F. (2014). Evidence of efficient stop codon readthrough in four mammalian genes. Nucleic Acids Research. https://doi.org/10.1093/nar/gku608

Lu, J., \& Deutsch, C. (2005). Folding zones inside the ribosomal exit tunnel. Nature Structural and Molecular Biology. https://doi.org/10.1038/nsmb1021

Lu, J., \& Deutsch, C. (2008). Electrostatics in the Ribosomal Tunnel Modulate Chain Elongation Rates. Journal of Molecular Biology. https://doi.org/10.1016/j.jmb.2008.08.089

Lu, J., Hua, Z., Kobertz, W. R., \& Deutsch, C. (2011). Nascent peptide side chains induce rearrangements in distinct locations of the ribosomal tunnel. Journal of Molecular Biology, 411(2), 499-510. https://doi.org/10.1016/j.jmb.2011.05.038

Maldonado, R., \& Herr, A. J. (1998). Efficiency of T4 gene 60 translational bypassing. Journal of Bacteriology, 180(7), 1822-1830.

Manktelow, E., Shigemoto, K., \& Brierley, I. (2005). Characterization of the frameshift signal of Edr, a mammalian example of programmed -1 ribosomal frameshifting. Nucleic Acids Research. https://doi.org/10.1093/nar/gki299

Maracci, C., \& Rodnina, M. V. (2016). Review: Translational GTPases. Biopolymers, 105(8), 463-475. https://doi.org/10.1002/bip.22832

Matsumoto, S., Caliskan, N., Rodnina, M. V., Murata, A., \& Nakatani, K. (2018). Small synthetic molecule-stabilized RNA pseudoknot as an activator for -1 ribosomal frameshifting. Nucleic Acids Research, 46(16), 8079-8089. https://doi.org/10.1093/nar/gky689

Melnikov, S., Ben-Shem, A., Garreau De Loubresse, N., Jenner, L., Yusupova, G., \& Yusupov, M. (2012). One core, two shells: Bacterial and eukaryotic ribosomes. Nature Structural and Molecular Biology, 19(6), 560-567. https://doi.org/10.1038/nsmb.2313

Miettinen, T. P., \& Bjorklund, M. (2015). Modified ribosome profiling reveals high abundance of ribosome protected mRNA fragments derived from 3' untranslated regions. Nucleic Acids Research. https://doi.org/10.1093/nar/gku1310

Milon, P., Konevega, A. L., Peske, F., Fabbretti, A., Gualerzi, C. O., \& Rodnina, M. V. (2007). Transient Kinetics, Fluorescence, and FRET in Studies of Initiation of Translation in Bacteria. In Methods in Enzymology. https://doi.org/10.1016/S0076$6879(07) 30001-3$ 
Milón, P., \& Rodnina, M. V. (2012). Kinetic control of translation initiation in bacteria. Critical Reviews in Biochemistry and Molecular Biology, 47(4), 334-348. https://doi.org/10.3109/10409238.2012.678284

Naganuma, T., Nomura, N., Yao, M., Mochizuki, M., Uchiumi, T., \& Tanaka, I. (2010). Structural basis for translation factor recruitment to the eukaryotic/archaeal ribosomes. Journal of Biological Chemistry, 285(7), 4747-4756. https://doi.org/10.1074/jbc.M109.068098

Nakatogawa, H., \& Ito, K. (2002). The ribosomal exit tunnel functions as a discriminating gate. Cell. https://doi.org/10.1016/S0092-8674(02)00649-9

Nissen, P., Hansen, J., Ban, N., Moore, P. B., \& Steitz, T. A. (2000). The structural basis of ribosome activity in peptide bond synthesis. Science. https://doi.org/10.1126/science.289.5481.920

Noeske, J., Huang, J., Olivier, N. B., Giacobbe, R. A., Zambrowski, M., \& Cate, J. H. D. (2014). Synergy of streptogramin antibiotics occurs independently of their effects on translation. Antimicrobial Agents and Chemotherapy. https://doi.org/10.1128/AAC.03389-14

Nosek, J., Tomaska, L., Burger, G., \& Lang, B. F. (2015). Programmed translational bypassing elements in mitochondria: Structure, mobility, and evolutionary origin. Trends in Genetics, 31(4), 187-194. https://doi.org/10.1016/j.tig.2015.02.010

Peng, B.Z., Bock, L.V., Belardinelli, R., Peske, F., Grubmüller, H. and Rodnina, M.V. (2019). Active role of elongation factor $G$ in maintaining the mRNA reading frame during translation. Science Advances (in press)

Peske, F., Rodnina, M. V., \& Wintermeyer, W. (2005). Sequence of steps in ribosome recycling as defined by kinetic analysis. Molecular Cell.

https://doi.org/10.1016/j.molcel.2005.04.009

Pisarev, A. V., Kolupaeva, V. G., Yusupov, M. M., Hellen, C. U. T., \& Pestova, T. V. (2008). Ribosomal position and contacts of mRNA in eukaryotic translation initiation complexes. EMBO Journal, 27(11), 1609-1621. https://doi.org/10.1038/emboj.2008.90

Pósfai, G., Plunkett, G., Fehér, T., Frisch, D., Keil, G. M., Umenhoffer, K., ... Blattner, F. R. (2006). Emergent properties of reduced-genome Escherichia coli. Science. https://doi.org/10.1126/science.1126439

Pulk, A., \& Cate, J. H. D. (2013). Control of ribosomal subunit rotation by elongation factor G. Science, 340(6140). https://doi.org/10.1126/science.1235970

Qin, P., Yu, D., Zuo, X., \& Cornish, P. V. (2014). Structured mRNA induces the ribosome into a hyper-rotated state. EMBO Reports, 15(2), n/a-n/a. https://doi.org/10.1002/embr.201337762

Qu, X., Wen, J. Der, Lancaster, L., Noller, H. F., Bustamante, C., \& Tinoco, I. (2011). The ribosome uses two active mechanisms to unwind messenger RNA during translation. Nature. https://doi.org/10.1038/nature10126 
References

Ramakrishnan, V. (2002). Ribosome structure and the mechanism of translation. Cell. https://doi.org/10.1016/S0092-8674(02)00619-0

Richter, D. (1976). Stringent factor from Escherichia coli directs ribosomal binding and release of uncharged tRNA. Proceedings of the National Academy of Sciences of the United States of America, 73(3), 707-711. https://doi.org/10.1073/pnas.73.3.707

Rodnina, M. V., Semenkov, Y. P., \& Wintermeyer, W. (1994). Purification of fMETtRNAfMET by Fast Protein Liquid Chromatography. Analytical Biochemistry. https://doi.org/10.1006/abio.1994.1282

Rodnina, Marina V. (2016). The ribosome in action: Tuning of translational efficiency and protein folding. Protein Science. https://doi.org/10.1002/pro.2950

Rodnina, Marina V. (2018). Translation in prokaryotes. Cold Spring Harbor Perspectives in Biology, 10(9). https://doi.org/10.1101/cshperspect.a032664

Rodnina, Marina V., Peske, F., Peng, B. Z., Belardinelli, R., \& Wintermeyer, W. (2019). Converting GTP hydrolysis into motion: Versatile translational elongation factor $\mathrm{G}$. Biological Chemistry. https://doi.org/10.1515/hsz-2019-0313

Rodnina, Marina V., Savelsbergh, A., Katunin, V. I., \& Wintermeyer, W. (1997). Hydrolysis of GTP by elongation factor $G$ drives tRNA movement on the ribosome. Nature, 385(6611), 37-41. https://doi.org/10.1038/385037a0

Rodnina, Marina V., Savelsbergh, A., Matassova, N. B., Katunin, V. I., Semenkov, Y. P., \& Wintermeyer, W. (1999). Thiostrepton inhibits the turnover but not the GTPase of elongation factor $\mathrm{G}$ on the ribosome. Proceedings of the National Academy of Sciences of the United States of America. https://doi.org/10.1073/pnas.96.17.9586

Rodnina, Marina V., \& Wintermeyer, W. (1995). GTP consumption of elongation factor Tu during translation of heteropolymeric mRNAs. Proceedings of the National Academy of Sciences of the United States of America. https://doi.org/10.1073/pnas.92.6.1945

Rodnina, Marina V., \& Wintermeyer, W. (2009). Recent mechanistic insights into eukaryotic ribosomes. Current Opinion in Cell Biology, 21(3), 435-443. https://doi.org/10.1016/j.ceb.2009.01.023

Rodnina, Marina V, Korniy, N., Klimova, M., Karki, P., Peng, B.-Z., Senyushkina, T., ... Peske, F. (2019). Translational recoding: canonical translation mechanisms reinterpreted. Nucleic Acids Research. https://doi.org/10.1093/nar/gkz783

Rozov, A., Khusainov, I., El Omari, K., Duman, R., Mykhaylyk, V., Yusupov, M., ... Yusupova, G. (2019). Importance of potassium ions for ribosome structure and function revealed by long-wavelength $\mathrm{X}$-ray diffraction. Nature Communications, 10(1), 1-12. https://doi.org/10.1038/s41467-019-10409-4

Saiki, R. K., Gelfand, D. H., Stoffel, S., Scharf, S. J., Higuchi, R., Horn, G. T., ... Erlich, H. A. (1988). Primer-directed enzymatic amplification of DNA with a thermostable DNA polymerase. Science, 239(4839), 487-491. 
https://doi.org/10.1126/science.2448875

Salsi, E., Farah, E., Netter, Z., Dann, J., \& Ermolenko, D. N. (2015). Movement of elongation factor $\mathrm{G}$ between compact and extended conformations. Journal of Molecular Biology, 427(2), 454-467. https://doi.org/10.1016/j.jmb.2014.11.010

Samatova, E., Konevega, A. L., Wills, N. M., Atkins, J. F., \& Rodnina, M. V. (2014). High-efficiency translational bypassing of non-coding nucleotides specified by mRNA structure and nascent peptide. Nature Communications, 5, 1-10. https://doi.org/10.1038/ncomms5459

Savelsbergh, A., Katunin, V. I., Mohr, D., Peske, F., Rodnina, M. V., \& Wintermeyer, W. (2003). An Elongation Factor G-Induced Ribosome Rearrangement Precedes tRNA-mRNA Translocation. Molecular Cell, 11(6), 1517-1523. https://doi.org/10.1016/S1097-2765(03)00230-2

Savelsbergh, A., Matassova, N. B., Rodnina, M. V., \& Wintermeyer, W. (2000). Role of domains 4 and 5 in elongation factor $\mathrm{G}$ functions on the ribosome. Journal of Molecular Biology. https://doi.org/10.1006/jmbi.2000.3886

Scarlett, D. J. G., McCaughan, K. K., Wilson, D. N., \& Tate, W. P. (2003). Mapping functionally important motifs SPF and GGQ of the decoding release factor RF2 to the Escherichia coli ribosome by hydroxyl radical footprinting: Implications for macromolecular mimicry and structural changes in RF2. Journal of Biological Chemistry. https://doi.org/10.1074/jbc.M211024200

Schägger, H. (2006). Protocol: Tricine-SDS-PAGE. Nature Protocols. https://doi.org/10.1038/nprot.2006.4

Schägger, H., \& von Jagow, G. (1987). Tricine-sodium dodecyl sulfate-polyacrylamide gel electrophoresis for the separation of proteins in the range from 1 to $100 \mathrm{kDa}$. Analytical Biochemistry, 166(2), 368-379. https://doi.org/10.1016/00032697(87)90587-2

Schluenzen, F., Tocilj, A., Zarivach, R., Harms, J., Gluehmann, M., Janell, D., ... Yonath, A. (2000). Structure of functionally activated small ribosomal subunit at 3.3 Å resolution. Cell. https://doi.org/10.1016/S0092-8674(00)00084-2

Schuwirth, B. S., Borovinskaya, M. A., Hau, C. W., Zhang, W., Vila-Sanjurjo, A., Holton, J. M., \& Cate, J. H. D. (2005). Structures of the Bacterial Ribosome at $3.5 \AA$ Resolution. Science, 310(5749), 827-834. https://doi.org/10.1126/science.1117230

Scolnick, E., Tompkins, R., Caskey, T., \& Nirenberg, M. (1968). Release factors differing in specificity for terminator codons. Proceedings of the National Academy of Sciences of the United States of America, 61(2), 768-774. https://doi.org/10.1073/pnas.61.2.768

Seidelt, B., Innis, C. A., Wilson, D. N., Gartmann, M., Armache, J.-P., Villa, E., ... Beckmann, R. (2009). Structural Insight into Nascent Polypeptide Chain-Mediated Translational Stalling. Science, 326(5958), 1412-1415. https://doi.org/10.1126/science.1177662 
References

Seidman, J. S., Janssen, B. D., \& Hayes, C. S. (2011). Alternative fates of paused ribosomes during translation termination. Journal of Biological Chemistry, 286(36), 31105-31112. https://doi.org/10.1074/jbc.M111.268201

Selmer, M., Gao, Y. G., Weixlbaumer, A., \& Ramakrishnan, V. (2012). Ribosome engineering to promote new crystal forms. Acta Crystallographica Section D: Biological Crystallography. https://doi.org/10.1107/S0907444912006348

Sharma, A. K., Sormanni, P., Ahmed, N., Ciryam, P., Friedrich, U. A., Kramer, G., \& O'Brien, E. P. (2019). A chemical kinetic basis for measuring translation initiation and elongation rates from ribosome profiling data. PLOS Computational Biology. https://doi.org/10.1371/journal.pcbi.1007070

Sharma, H., Adio, S., Senyushkina, T., Belardinelli, R., Peske, F., \& Rodnina, M. V. (2016). Kinetics of Spontaneous and EF-G-Accelerated Rotation of Ribosomal Subunits. Cell Reports. https://doi.org/10.1016/j.celrep.2016.07.051

Shatsky, I. N., Terenin, I. M., Smirnova, V. V., \& Andreev, D. E. (2018). CapIndependent Translation: What's in a Name? Trends in Biochemical Sciences, 43(11), 882-895. https://doi.org/10.1016/j.tibs.2018.04.011

Skuzeski, J. M., Nichols, L. M., Gesteland, R. F., \& Atkins, J. F. (1991). The signal for a leaky UAG stop codon in several plant viruses includes the two downstream codons. Journal of Molecular Biology. https://doi.org/10.1016/0022-2836(91)90718$\mathrm{L}$

Smith, A. M., Costello, M. S., Kettring, A. H., Wingo, R. J., \& Moore, S. D. (2019). Ribosome collisions alter frameshifting at translational reprogramming motifs in bacterial mRNAs. Proceedings of the National Academy of Sciences. https://doi.org/10.1073/pnas. 1910613116

Sonenberg, N., \& Hinnebusch, A. G. (2009). Regulation of Translation Initiation in Eukaryotes: Mechanisms and Biological Targets. Cell, 136(4), 731-745. https://doi.org/10.1016/j.cell.2009.01.042

Steitz, T. A. (2008). A structural understanding of the dynamic ribosome machine. Nature Reviews Molecular Cell Biology, 9(3), 242-253. https://doi.org/10.1038/nrm2352

Takyar, S., Hickerson, R. P., \& Noller, H. F. (2005). mRNA helicase activity of the ribosome. Cell, 120(1), 49-58. https://doi.org/10.1016/j.cell.2004.11.042

Thommen, M., Holtkamp, W., \& Rodnina, M. V. (2017). Co-translational protein folding: progress and methods. Current Opinion in Structural Biology. https://doi.org/10.1016/j.sbi.2016.11.020

Tiller, N., \& Bock, R. (2014). The translational apparatus of plastids and its role in plant development. Molecular Plant. https://doi.org/10.1093/mp/ssu022

Todd, G. C., \& Walter, N. G. (2013). Secondary structure of bacteriophage T4 gene 60 mRNA: Implications for translational bypassing. RNA.

https://doi.org/10.1261/rna.037291.112 
Tork, S., Hatin, I., Rousset, J. P., \& Fabret, C. (2004). The major 5' determinant in stop codon read-through involves two adjacent adenines. Nucleic Acids Research. https://doi.org/10.1093/nar/gkh201

Trovato, F., \& O'Brien, E. P. (2017). Fast Protein Translation Can Promote Coand Posttranslational Folding of Misfolding-Prone Proteins. Biophysical Journal, 112(9), 1807-1819. https://doi.org/10.1016/j.bpj.2017.04.006

Tsai, A., Kornberg, G., Johansson, M., Chen, J., \& Puglisi, J. D. (2014). The dynamics of SecM-induced translational stalling. Cell Reports, 7(5), 1521-1533. https://doi.org/10.1016/j.celrep.2014.04.033

Ude, S., Lassak, J., Starosta, A. L., Kraxenberger, T., Wilson, D. N., \& Jung, K. (2013). Translation elongation factor EF-P alleviates ribosome stalling at polyproline stretches. Science. https://doi.org/10.1126/science.1228985

Voorhees, R. M., \& Ramakrishnan, V. (2013). Structural Basis of the Translational Elongation Cycle. Annual Review of Biochemistry, 82(1), 203-236. https://doi.org/10.1146/annurev-biochem-113009-092313

Wang, X., Xuan, Y., Han, Y., Ding, X., Ye, K., Yang, F., ... Gao, G. (2019). Regulation of HIV-1 Gag-Pol Expression by Shiftless, an Inhibitor of Programmed -1 Ribosomal Frameshifting. Cell. https://doi.org/10.1016/j.cell.2018.12.030

Weinberg, D. E., Shah, P., Eichhorn, S. W., Hussmann, J. A., Plotkin, J. B., \& Bartel, D. P. (2016). Improved Ribosome-Footprint and mRNA Measurements Provide Insights into Dynamics and Regulation of Yeast Translation. Cell Reports. https://doi.org/10.1016/j.celrep.2016.01.043

Weiss, R. B., Huang, W. M., \& Dunn, D. M. (1990). A nascent peptide is required for ribosomal bypass of the coding gap in bacteriophage T4 gene 60. Cell, 62(1), 117126. https://doi.org/10.1016/0092-8674(90)90245-A

Wilden, B., Savelsbergh, A., Rodnina, M. V., \& Wintermeyer, W. (2006). Role and timing of GTP binding and hydrolysis during EF-G-dependent tRNA translocation on the ribosome. Proceedings of the National Academy of Sciences of the United States of America. https://doi.org/10.1073/pnas.0606099103

Williams, I., Richardson, J., Starkey, A., \& Stansfield, I. (2004). Genome-wide prediction of stop codon readthrough during translation in the yeast Saccharomyces cerevisiae. Nucleic Acids Research. https://doi.org/10.1093/nar/gkh1004

Wills, N. M., O'Connor, M., Nelson, C. C., Rettberg, C. C., Huang, W. M., Gesteland, R. F., \& Atkins, J. F. (2008). Translational bypassing without peptidyl-tRNA anticodon scanning of coding gap mRNA. EMBO Journal, 27(19), 2533-2544. https://doi.org/10.1038/emboj.2008.170

Wilson, D. N., Arenz, S., \& Beckmann, R. (2016). Translation regulation via nascent polypeptide-mediated ribosome stalling. Current Opinion in Structural Biology, 37, 123-133. https://doi.org/10.1016/j.sbi.2016.01.008

Wimberly, B. T., Brodersen, D. E., Clemons, W. M., Morgan-Warren, R. J., Carter, A. P., 
References

Vonrheln, C., ... Ramakrishnan, V. (2000). Structure of the 30 S ribosomal subunit. Nature, 407(6802), 327-339. https://doi.org/10.1038/35030006

Wintermeyer, W. (1994). Transient conformational states of aminoacyl-tRNA during ribosome binding catalyzed by elongation factor Tu. Biochemistry. https://doi.org/10.1021/bi00206a033

Wohlgemuth, I., Brenner, S., Beringer, M., \& Rodnina, M. V. (2008). Modulation of the rate of peptidyl transfer on the ribosome by the nature of substrates. Journal of Biological Chemistry. https://doi.org/10.1074/jbc.M805316200

Wohlgemuth, I., Pohl, C., \& Rodnina, M. V. (2010). Optimization of speed and accuracy of decoding in translation. EMBO Journal. https://doi.org/10.1038/emboj.2010.229

Woolhead, C. A., McCormick, P. J., \& Johnson, A. E. (2004). Nascent membrane and secretory proteins differ in FRET-detected folding far inside the ribosome and in their exposure to ribosomal proteins. Cell. https://doi.org/10.1016/S00928674(04)00169-2

Yan, S., Wen, J. Der, Bustamante, C., \& Tinoco, I. (2015). Ribosome Excursions during mRNA Translocation Mediate Broad Branching of Frameshift Pathways. Cell. https://doi.org/10.1016/j.cell.2015.02.003

Yao, L., Li, Y., Tsai, T. W., Xu, S., \& Wang, Y. (2013). Noninvasive measurement of the mechanical force generated by motor protein EF-G during ribosome translocation. Angewandte Chemie - International Edition, 52(52), 14041-14044. https://doi.org/10.1002/anie.201307419

Yusupov, M. M., Yusupova, G. Z., Baucom, A., Lieberman, K., Earnest, T. N., Cate, J. H. D., \& Noller, H. F. (2001). Crystal structure of the ribosome at $5.5 \AA$ resolution. Science, 292(5518), 883-896. https://doi.org/10.1126/science.1060089

Yusupova, G. Z., Yusupov, M. M., Cate, J. H. D., \& Noller, H. F. (2001). The path of messenger RNA through the ribosome. Cell. https://doi.org/10.1016/S00928674(01)00435-4

Zaher, H. S., \& Green, R. (2011). A primary role for release factor 3 in quality control during translation elongation in Escherichia coli. Cell, 147(2), 396-408. https://doi.org/10.1016/j.cell.2011.08.045

Zhang, J., Pan, X., Yan, K., Sun, S., Gao, N., \& Sui, S. F. (2015). Mechanisms of ribosome stalling by SecM at multiple elongation steps. ELife, 4, 1-25. https://doi.org/10.7554/eLife.09684

Zhang, W., Dunkle, J. A., \& Cate, J. H. D. (2009). Structures of the ribosome in intermediate states of ratcheting. Science, 325(5943), 1014-1017. https://doi.org/10.1126/science.1175275

Zhang, Y., Baranov, P. V., Atkins, J. F., \& Gladyshev, V. N. (2005). Pyrrolysine and selenocysteine use dissimilar decoding strategies. Journal of Biological Chemistry. https://doi.org/10.1074/jbc.M501458200 
Zheng, X., Hu, G. Q., She, Z. S., \& Zhu, H. (2011). Leaderless genes in bacteria: Clue to the evolution of translation initiation mechanisms in prokaryotes. $B M C$

Genomics, 12(1), 361. https://doi.org/10.1186/1471-2164-12-361

Zhou, J., Lancaster, L., Donohue, J. P., \& Noller, H. F. (2013). Crystal structures of EF$\mathrm{G}$ - Ribosome complexes trapped in intermediate states of translocation. Science. https://doi.org/10.1126/science.1236086 
